\title{
TWO ESSAYS ON NEW B2B SALES INCENTIVE MECHANISMS FOR ENHANCING SELLING EFFECTIVENESS AND COORDINATING INSIDE AND OUTSIDE SALES FORCES
}

\author{
A Dissertation \\ presented to \\ the Faculty of the Graduate School \\ at the University of Missouri-Columbia
}

In Partial Fulfillment

of the Requirements for the Degree

Doctor of Philosophy

\author{
by \\ YEJI LIM \\ Dr. Murali K. Mantrala, Dissertation Supervisor \\ DECEMBER 2019
}


The undersigned, appointed by the Dean of the Graduate School, have examined the dissertation entitled:

TWO ESSAYS ON NEW B2B SALES INCENTIVE MECHANISMS:

FOR ENHANCING SELLING EFFECTIVENESS

AND COORDINATING INSIDE AND OUTSIDE SALES FORCES

presented by Yeji Lim,

a candidate for the degree of Doctor of Philosophy

and hereby certify that, in their opinion, it is worthy of acceptance.

Professor Murali K. Mantrala

Professor Detelina Marinova

Professor Syam B. Niladri

Professor Ashutosh Patil

Professor Oksana Loginova 
This dissertation is dedicated to my beloved Kyungsik Nam. 


\section{ACKNOWLEDGMENTS}

My five year journey to earn a Ph.D. has culminated in this dissertation. Several precious individuals and entities have played a significant role in my journey to this point.

First, I wish to acknowledge my dissertation advisor and mentor, Professor Murali Mantrala. Without his advice, I could not have started and finished these ambitious papers. He always encouraged me to think out of the box by sharing his invaluable experience and opinions which formed the foundation for my research. He was the reason I chose the University of Missouri and it was a great honor for me to have a chance to work with him. His guidance has launched my academic career.

I also would like to express my appreciation to my other committee members, Dr. Detelina Marinova, Dr. Syam Niladri, Dr. Ashutosh Patil, and Dr. Oksana Loginova for their astute observations, thoughtful comments, and suggestions. While I've been through hardships during the doctoral program, their friendly advice, encouragement, and support were also my mainstays in my doctoral research.

Many thanks also to Dr. Lisa Scheer for teaching me how to effectively communicate my research to marketing audiences. I could have not improved without her sharp comments on writing and speaking communication skills as a scholar. I also wish to thank Surya Mantrala and Nivedita Mukherjee for treating me like a part of their families.

I owe a lot also to my good doctoral student colleagues and friends, Dr. Anna Kim, Dr.Shinhye Kim, Divya Anand and Bitty Balduchi. They always have been willing to give me their best suggestions and emotional support. Above all, I would like to thank my parents. My mother, Bocksoon Cho, my mother-in-law, Kyungsun Park, my father, Iksoon Lim, and my father-in-law Dongwoo Nam have supported and encouraged me always to continue my doctoral work. Lastly, I am deeply grateful 
to my wonderful husband, Kyungsik Nam for his endless support, encouragement, and prayers. Without his support, I could not even start my Ph.D. study. He motivated and encouraged me at the most frustrating moments. I would like to dedicate this dissertation to him.

Yeji Lim in the Cornell Hall Office 


\section{TABLE OF CONTENTS}

ACKNOWLEDGMENTS

LIST OF TABLES vi

LIST OF FIGURES vii

ABSTRACT viii

Chapters

I. OVERVIEW 1

1. Overview 1

2. Motivating Salespeople to Enhance Selling Effectiveness 2

3. Coordinating Inside and Outside Sales Forces 2

II. ESSAY 1:

$\begin{array}{ll}\text { 1. Introduction } & 7\end{array}$

2. Literature Review 11

3. Extended Principal-Agent model 15

4. Alternative Compensation Plan Scenarios 19

5. Numerical Simulation Analyses 41

6. Conclusion 46

$\begin{array}{ll}\text { III. ESSAY 2: } & 49\end{array}$

1. Introduction 49

2. Literature Review $\quad 51$

3. Target-Value Compensation Plan 55

4. Optimal Solutions 62

5. Extension: Comparison with Alternative Compensation Plans 68 
1. Proposition Proofs of Essay1 87

2. Proposition Proofs of Essay2 101

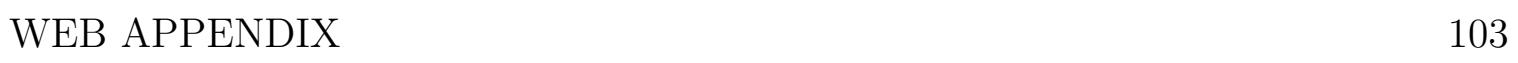

1. Comparative Statics Proofs for Essay 1 103

2. Optimization of New Model with Adaptable Selling Effectiveness 109

3. Comparative Statics Proofs for Essay $2 \quad 114$

4. Optimization of Target-Value Compensation 115 


\section{List of Tables}

1 Literature Review . . . . . . . . . . . . . . . . . . 11

2 List of Symbols . . . . . . . . . . . . . . . . . . . . . 14

3 Four Principal-Heterogeneous Agent Model Solutions . . . . . . . . . 19

4 Comparative Statics in Standard-Customized commission . . . . . . . 22

5 Comparative Statics in Multidimensional-Customized commission . . 28

6 Empirical Mean of Parameters from Daljord et al. (2016) . . . . . . . 40

$7 \quad$ List of Symbols . . . . . . . . . . . . . . . . . 55

8 Salespeople's Choices Associated with Incentives . . . . . . . . . . . . 61

9 Optimal Wage Parameters .................. 62

10 Salespeople's Optimal Choices . . . . . . . . . . . . . . 63

11 Comparative Statics for ISR . . . . . . . . . . . . . 65

12 Comparative Statics for OSR . . . . . . . . . . . 65

13 Salespeople's Choices Associated with Incentives Under Alternative Plans 69

14 Optimal Wage Parameters under Alternative Plans . . . . . . . . . . 69

15 Empirical Mean of Parameters for Numerical Simulation . . . . . . . 71

16 Simulation Comparison under the three different plans . . . . . . . . 72 


\section{List of Figures}

1 Sequence of move between firms and salespeople . . . . . . . . . . . . 17

2 Types of Compensation Plans . . . . . . . . . . . . . . . . . . 19

3 Comparison of Selling Effectiveness in Traditional model $\beta_{t r d}$ and in

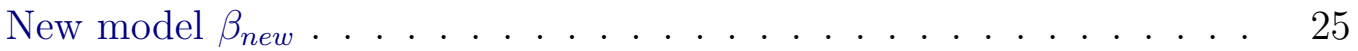

4 Comparison based on Heterogeneous Basic Ability between Traditional and New Model . . . . . . . . . . . . . . . . . . . . . 41

5 Cumulative Comparison between Traditional and New Model . . . . . 41

6 Comparison between Standard and Multidimensional Compensation . 43

$7 \quad$ Firm's Profit along with Sales Level . . . . . . . . . . . . . . . . . . . 72 


\section{ABSTRACT}

\section{Essay 1: Motivating Salespeople to Enhance Selling Effectiveness: The Role and Optimal Design of Activity-Based Incentive Plans}

This paper provides a rationale and model for the use and optimal design of salesplus activity-based incentive plans that are quite common in sales management practice. So far, there is no theoretical research providing guidance on why and how to structure such mechanisms. To meet this need, I argue that the role of appropriate activity-based sales force incentives is to increase selling effectiveness just like the usual sales commissions are utilized for motivating higher selling effort. I conceptualize that enhanced selling effectiveness stems from greater thinking efforts in the preparation for as well as execution of customer sales calls, and appropriately selected activities induce such thinking efforts. By relaxing the assumption of fixed selling effectiveness that is present in all agency-theoretic sales force incentive models to date, and allowing for adaptable selling effectiveness and sales that are a function of both selling and thinking efforts, I derive and compare optimal sales plus activity-based incentive plans with only sales-based incentives. I establish the conditions under which the former multidimensional plans produce outcomes for both the firm and the salesperson that are superior, as well as provide guidance for their operational design in practice.

\section{Essay 2: Compensation Mechanism for Coordinating Inside and Outside Sales Forces}

To ensure cooperation between inside and outside salespeople of business-tobusiness (B2B) sales organizations I propose and analytically demonstrate the benefits of a new joint compensation system. This proposed new compensation system is designed to facilitate the exchange of well-qualified leads between inside and outside salespeople. The motivation for designing this new system is the Internet-induced 
fundamental changes in $\mathrm{B} 2 \mathrm{~B}$ buyers purchasing behavior from a reactive or sellerdriven to a proactive or buyer-driven approach. These trends have led to inside sales forces rising in importance and value relative to outside sales forces in B2B sales. However, they pose problems to B2B selling firms: 1) dissonance between inside and outside salespeople; 2) loss of potential customers as each sales group pursues those leads of most relevance to the group; and 3) increasing costs when each group passes on poorly qualified leads to the other group. To mitigate these problems, my proposed compensation mechanism ties each sales groups incentives to achievement against self-selected targets for leads to be passed on to the other group. In this research, I describe and investigate the impacts of properties of this compensation plan and also compare its impact on salespeoples utilities and earnings, and firms profits, with those of two other incentive mechanisms (i) A system that rewards salespeople for their own performance; and (ii) A system under which salespeople are partly rewarded for corporate performance but receive no extra incentive for exchanging leads. My simulation results show that the target-value compensation system is optimal for the firm and salespeople. More specifically, the target-value compensation system ensures that (1) salespeople of one group will endeavor to deliver well-qualified leads to the other side and (2) firms do not need to observe sales forces actions. 


\section{CHAPTER I: OVERVIEW}

\section{Overview}

\subsection{Overview}

As the Internet Age has advanced, B2B buyers and process have also evolved. Smarter buyers have proactively been searching for and using information on the Internet to compile, compare and choose between alternatives. Also, the B2B purchasing journey has become shorter than before. Hence, more nimble-footed sellers have adopted a high-tech marketing strategy with machine learning and big data to track their consumers present preferences and offer customized product offerings or solutions. Following this trend, sales organizations are also compelled to enhance their sales reps' selling effectiveness and shift from persuading to guiding inbound B2B buyers in their purchasing process.

One strategy to enhance sales reps selling effectiveness is hire the right people and invest heavily in training each rep to become a more effective seller. A second strategy that I explore in my dissertation Essay 1 is to optimally design a sales - plus activity-based incentive mechanism to motivate reps to improve their selling effectiveness. I do so by positing a new sales response model, which allows for adaptive selling effectiveness for the first time in the literature, where sales are a function of both thinking and selling efforts. With this new assumption I examine and compare performance under standard sales-based commission mechanism with a sales- plus activity-based 'multidimensional' incentive mechanism and establish the conditions when the latter provides superior benefits to the rep as well as the firm.

In Essay 2, I shift my attention to the problem of coordinating the efforts of 
traditional outside sales force and an emerging inside sales force organization at many B2B firms. Since more well-informed buyers bypass outside salespeople and approach sellers' inside salespeople for guidance on the right solutions to their needs, new inside sales reps have become much more actively responsible for developing as well as closing deals. However, outside salespeople are also needed to engage with other buyers at different stages of their buying process. Therefore, as sellers are compelled to develop both outside and inside sales organizations, their coordination becomes critical to close and keep satisfied customers. In essay 2, I propose a target-value compensation scheme to motivate such coordination in handling of new potential customer leads that come in via both outbound and inbound marketing by sellers..

\subsection{Motivating Salespeople to Enhance Selling Effectiveness}

As selling environments have become more complex and competitive, B2B marketers and consultants are placing increasing emphasis on enhancing their salespeople's selling effectiveness. Moorman et al. (2012) report that this goal is the topmost priority for many Fortune 500 companies' executives as data indicate that improvements in selling effectiveness alone can raise profitable organic growth by 5-20\%.

Following Szymanski (1988), I define 'selling effectiveness' here as the salesperson's ability to identify and classify accurately the target client's product- and sellingrelated needs at different stages of the sales process (prospecting, sales call, sales presentation, and sale closing). Differences in selling effectiveness explain variations in resulting sales performance. A key determinant of greater selling effectiveness is better organized declarative knowledge (facts or information stored in memory) that will enable more accurate categorization of a client's needs at each selling stage by the salesperson (Szymanski 1988). A second determinant is the amount of cognitive or thinking effort put into researching and processing incoming data on prospective customers so as to accurately categorize them in terms of their needs in different sell- 
ing stages. In this regard, there is evidence that more deliberation, i.e., more thinking effort is more effective than less thinking effort, or intuitive thinking, e.g., Kahneman et al. (2005), Moxley et al. (2012). Given the increasing complexity of selling environments today, interest and investments in sales training programs aimed at enhancing selling effectiveness via declarative knowledge development as well as critical thinking skills development are considerable (Alvarez et al. 2015). Sales training investments in the US alone are annually over $\$ 15$ billion according to LinkedIn's State of Sales Report in 2018.

Firms also spend over $\$ 800$ billion on sales incentive plans. However, much of the latter is spent on purely output-based incentives that tend to focus sales reps' attention on increasing selling effort without necessarily improving selling effectiveness. But, in many B2B markets today, the need of the hour is greater selling effectiveness more than greater selling effort to engage with much more well-informed but timepressed organizational buyers with heterogeneous needs (Paesbrugghe et al. 2017). Focusing on the issue of enhancing selling effectiveness, while acknowledging that periodic training is important, in this paper I consider the following question: Can the sales force incentive plan itself be designed to induce a sales rep to strive to be more effective in her/his sales calls, i.e., work smarter, as well as increase his/her selling effort, i.e., work harder? By building and analyzing a new model extension of the classic principal-agent (PA) model of sales force compensation (e.g., Basu et al. (1985), Lal and Srinivasan (1993)), I establish that the answer to this question is affirmative and can be achieved by a 'multidimensional' plan that combines appropriate activity-based incentives with standard sales-based incentives.

\subsection{Coordinating Inside and Outside Sales Forces}

In 2017, InsideSales.com reported that $47.2 \%$ (2.7 million) out of 5.7 million are Inside sales reps in US Business-to-Business (B2B) firms and they expect that the 
number of inside reps would increase up to $50 \%$. Consequently, one of the biggest challenges for many B2B selling firms is how to coordinate and ensure Inside and Outside Salespeople work cooperatively even when they are separate sales teams (Arli et al. 2018).

As technology has developed, B2B buyers purchasing behavior has been changed from passive to active (Steward et al. 2019, Toman et al. 2017). These days, B2B buyers explore all necessary information proactively to purchase complex products by searching the internet, reading white papers/E-books, visiting Vendor Web sites, participating in Webinars, or checking social media (Toman et al. 2017, Mantrala and Albers 2012). Thus, B2B inside salespeople via search engines, blogs, and interactions with prospective buyers using social media are generating new business at higher rates (Grewal et al. 2015, Mantrala 2014). Also, Salesforce.com's report shows that Inside sales roles have increased up to $7 \%$ since 2015 (Salesforce.com). In contrast, the traditional outside salespeople's sales, via cold calling or trade shows, have diminishes because of B2B buyers' Internet usage (Steenburgh et al. 2009). Consequently, as Inside and Outside (e.g., field sales reps) sales reps' positions have become almost even in importance, their smooth coordination to provide a seamless front to inquiring customers has become a big issue for firms (Arli et al. 2018).

More specifically, two serious problems may sequentially arise in outside and inside salesperson activities. First, inside sales reps (ISR) and outside sales reps (OSR) may not cooperate. Traditionally, ISRs worked as administrative aides for OSRs (e.g., call centers, telemarketers) (Bendapudi and Leone 2002) and were not actively involved in prospecting or closing sales (Shi et al. 2017). Then, the ISR and OSR worked cooperatively as a hybrid team and sometimes shared team-incentives to motivate them to cooperate (Arli et al. 2018). However, as ISRs today play more independent sales role by remotely closing deals based on inbound buyers' contacts and information (Salesforce.com), they expect to receive their own sales-based incentives just like 
OSRs (Kundu 2019). Consequently, motivation for an ISR and OSR to cooperate has diminished. This brings up the second problem, namely, poor B2B customer experience with the sellers' multiple touchpoints.

Considering that firms' multiple touchpoints (e.g., electronic connections, inperson contact) affect potential consumers' purchasing journey and experience (Lemon and Verhoef 2016), those touchpoints with noncooperative multiple salespeople will dissatisfy potential customers. For instance, when a potential consumer who really prefers remote communication makes first contact with an OSR to ask about services and products, that OSR is not really the appropriate channel to determine and sell the right product to this customer. In this case, there are three ways for the OSR to respond. First, the OSR may abandon the potential consumer and focus on another potential consumer to increase his/her own sales earnings. The abandoned potential consumer will be disappointed and leave to find another firm (Strategies 2019). Second, the OSR may try to sell products him/herself remotely to the prospective consumer. However, OSR's sales may not be successful because s/he is not set up for or good at remote communication as much as the ISR is (Sincavage 2019). Third, the OSR can pass on the lead to the appropriate ISR, which is the preferred path for this potential consumer and result in a sale. Undoubtedly, the choice of this third option by the OSR will be the best outcome the firm, the buyer, and the ISR - provided the lead was somewhat qualified by the OSR.

However, the OSR is more likely to choose one of the first two options because there is no incentive for him/her to undertake the effort involved in passing on a reasonably qualified lead to the ISR. More specifically, the OSR does not receive any benefit for handing over the potential customer to the ISR under a standard compensation plan (e.g., salary plus incentive on sales). Moreover, s/he loses time to sell products to his/her own higher potential customers. Under the standard compensation plan, a similar problem can arise with an inbound customer lead that first goes to an ISR even 
though it becomes evident that the customer is best dealt with by an OSR. Overall, therefore, ensuring coordination between OSRs and ISRs that maximize customer sales, experience, and satisfaction requires a new form of joint incentive mechanism that motivates qualification and exchange of customer leads between the two sales forces. The question of how this new mechanism can be optimally structured is the focus of Essay 2. 
CHAPTER II: Essay 1

\section{Motivating Salespeople to Enhance Selling Effectiveness: The Role and Optimal Design of Activity-Based Incentive Plans}

\section{Introduction}

In general, activity-based incentives are in fact quite common in practice. Thus, most sales management textbooks include a section on the use of 'activity quotas' and monetary rewards for achieving them, e.g., bonuses or commissions tied to the number of calls made, emails sent, number of conversations, social media interactions, appointments scheduled, referral requests made, and proposals sent (Schreiner 2018). The trade press also contains many blogs and commentaries regarding the pros and cons of various key performance indicators (KPIs) that could be employed in output-based as well as activity-based selling incentive mechanisms (Chung et al. 2019, Zoltners et al. 2019). In the present article, I specifically focus on those activity measures termed 'leading indicators' of ultimate sales, e.g., Cespedes and Marsh (2017). These are measures of activities that are within a sales rep's control and are known drivers of sales (e.g., https://www.pipedrive.com/en/blog/activity-based-selling). Examples of such leading indicators include product 'demos', web registrations, new leads generated, or C-suite level meetings (Cespedes and Marsh 2017).

Consider, for instance, the 'number of demos', i.e., product demonstrations, completed by a salesperson during some defined accounting period. This is the archetypal leading indicator activity measure that I assume in my analysis of activity-based incentive plans aimed at boosting selling effectiveness and overall performance. This is 
because a product demonstration is a common, trackable input activity for evaluating salesperson performance (e.g., Jackson and Tax (1995)), known to be a relevant and valuable ingredient of effective adaptive selling (e.g., Delvecchio et al. (2004)), and a very targeted way to get your product in front of the right kind of customer (quote from Schembari (2007) in Heiman (2010)). More specifically, increasing the number of effective demos calls for the same thinking efforts that contribute to greater overall selling effectiveness. In a nutshell, the completion of more effective product demos is associated with greater selling effectiveness, e.g., DelVecchio et al. (2003). In contrast to such leading indicators, the typical 'lagging' metrics used in traditional sales force incentive plans, e.g., sales volume, number of sales orders, number of new or profitable customers etc, are all output-related and fall in the category of 'sales-based' metrics. Lastly, an activity metric like 'the number of calls' is an input activity metric but is a measure of 'selling effort' rather than 'selling effectiveness'. In short, hereafter, when I refer to 'activity-based' metrics I mean leading indicator activities such as product demos that are plausibly associated with input selling effectiveness as distinct from input selling effort or output sales.

So far, as already mentioned, the quantitative sales force incentives research literature has devoted hardly any attention to the rationale and optimal setting of activity-based incentives as a part of salespeople's regular compensation plan. The bulk of the quite substantial extant literature focuses on the design of salary plus sales output-based incentive plans using principal-agent (PA) models (see, e.g. Mantrala (2014) for a review). This is because, fundamentally, extant models have generally assumed selling effort and other input activity are unobservable (e.g., Holmstrom (1979), Basu et al. (1985)). Because of this unobservability of actions in uncertain selling environments, the literature naturally focused on 'second-best' sales outputbased incentive mechanisms. However, there are two reasons to give more emphasis to activity-based incentives today: First, the observability of an outside sales rep's in- 
put activities has grown in recent times as various technologies have made monitoring by management more feasible and less expensive than three decades ago when many of the PA models of sales force compensation design appeared. Second, as already noted, enhancing selling effectiveness is critical today and it is input activities that hone effectiveness that need to be stimulated. However, almost without exception, the previous PA literature (Basu et al. 1985, Lal and Srinivasan 1993, Mantrala et al. 1994, Albers 1996, Raju and Srinivasan 1996, Mishra and Prasad 2004, Daljord et al. 2016) assumes individual reps' selling effectiveness is constant or fixed, and focus on motivating reps to increase quantity of work (e.g., number of selling hours). Thus, this literature, by and large, has ignored the many empirical studies over the same period of time that have shown that a salesperson's selling effectiveness is variable and adaptable (Sujan et al. 1994, Rapp et al. 2006, Fang et al. 2008) and can be improved via behavior-based control mechanisms (Anderson and Oliver 1987, Cravens et al. 1993, Oliver and Anderson 1994).

Lastly, extant theoretical PA model papers have mostly investigated the optimal structuring of customized plans, i.e., plans with incentive parameters or terms that vary across heterogeneous salespeople (Basu et al. 1985, Lal and Srinivasan 1993, Raju and Srinivasan 1996, Mantrala et al. 1997, Joseph and Thevaranjan 1998, Kalra et al. 2003, Murthy and Mantrala 2005, Rubel and Prasad 2015, Schöttner 2016). In practice, however, compensation plans that are restricted to having a uniform commission rate for all salespeople are quite common, typically because they provide the semblance of equity and are easy and cheaper to implement (e.g., Mantrala et al. (1994)). Only recently, Daljord et al. (2016) have shown that such restricted uniform plans can be close to optimal if firms choose sales force composition and compensation at the same time. Now considering that the proposed multidimensional (MD) salesplus activity-based compensation plans in the present paper are likely to be even more expensive to administer on a customized basis, this begs the question of how well a 
uniform version of the MD plan performs relative to the corresponding customized version? The answer will have an important bearing on the use of such MD plans to raise selling effectiveness.

In summary, the existing classic sales force compensation literature has three major gaps from the perspective of motivating greater selling effectiveness: 1) No prior outcome-based compensation plan analysis allows for adaptable selling effectiveness along with variable selling effort; 2) No model for the optimal design of multidimensional (outcome- plus activity-based) compensation plan aimed at inducing higher selling effectiveness as well as greater selling effort has been proposed and investigated analytically; and 3) No comparative analyses and insights into aggregate firm performance under optimal sales-based versus multidimensional plans, either uniform or customized, have been provided to date.

To address the above gaps in the sales compensation literature and provide guidance with regard to use and structuring of multidimensional activity-based incentive plans, I propose for the first time an extended 'behavioral' principal-agent model that allows for adaptable selling effectiveness. I then solve this model for the optimal customized as well as uniform sales-based (or 'standard') and MD (sales- plus activitybased) commission plans, and compare the outcomes for the salesperson and the firm under these alternative incentive mechanisms. My three-fold objective of these analyses are to answer the following questions: When selling effectiveness is adaptable: (1) Can the proposed new multidimensional compensation mechanism, that rewards reps for both sales and effectiveness-enhancing activity, improve both reps' and the firm's outcomes? (2) How does sales force performance respond to either a uniform or customized standard sales commission-based incentive plan? (3) How does sales force performance respond to either a uniform or customized new MD incentive plan? Subsequently, my analyses show that both the salesperson as well as the firm can obtain greater benefit from using an optimally structured multidimensional incentive 
plan that indirectly controls both selling effectiveness as well as selling effort.

The rest of the paper is organized as follows. In the next section, I briefly review the key papers in agency-theoretic literature on sales force compensation plan. In section 3, I develop my proposed behavioral agency model and solve for an optimal firm and reps' actions and payoffs under four different compensation scenarios: Standard-Customized (SC), Standard-Uniform (SU), Multidimensional-Customized (MD-C), and Multidimensional-Uniform (MD-U) compensation plans. In section 4, I discuss the new propositions and insights emerging from the analyses of my model under the four compensation scenarios and in section 5, I show the results of numerical simulation analyses to illustrate my propositional results. Finally, the last section concludes with a summary of my research contributions, managerial implications and suggested directions for future research.

\section{Literature Review}

Understanding and designing compensation mechanisms that motivate greater sales force productivity and higher profits for the firm have been among the key issues of research in sales management for the last several decades, e.g., Farley (1964), Srinivasan (1981), Basu et al. (1985) (see Mantrala 2014 for a historical review). Since the mid-'80s, the dominant paradigm for understanding and designing optimal sales force incentive plans has been principal-agent theory from economics (e.g., Holmstrom and Milgrom 1987). As summarized in Table 1, the different sales incentive problems that have been addressed include the design of salary plus sales commission plans (Basu et al. 1985, Basu and Kalyanaram 1990); multi-product sales commission and quota-based bonus plans (Lal and Srinivasan 1993, Mantrala et al. 1994); optimal plan design under asymmetric information (Lal and Staelin 1986, Rao 1990); sales quotabased plans for eliciting honest sales forecasts (Gonik 1978, Mantrala and Raman 
1990); optimal incentives for salespeople with heterogeneous sales territories (Raju and Srinivasan 1996); optimal multi-person relative performance sales contests (Kalra et al. 2003, Murthy and Mantrala 2005, Syam et al. 2013); and optimal multi-period or dynamic sales incentive plans (Dearden and Lilien 1990, Misra and Nair 2011, Rubel and Prasad 2015). Empirical tests of analytical model findings have also been conducted using various types of data to validate propositions from PA models (John and Weitz 1989, Coughlan and Narasimhan 1992, Misra et al. 2005, Chung et al. 2013, Daljord et al. 2016).

Table 1: Literature Review

\begin{tabular}{|c|c|c|c|c|c|c|c|c|}
\hline & & \multicolumn{2}{|c|}{ Selling Effectiveness } & \multicolumn{2}{|c|}{ Effort } & \multicolumn{3}{|c|}{ Incentive on } \\
\hline & & Fixed & Adaptable & Selling & Thinking & Sales & Activity & Sales+Activity \\
\hline 1 & Gonik (1978) & $\checkmark$ & & $\checkmark$ & & $\checkmark$ & & \\
\hline 2 & Srinivasan (1981) & $\checkmark$ & & $\checkmark$ & & $\checkmark$ & & \\
\hline 3 & Basu et al. (1985) & $\checkmark$ & & $\checkmark$ & & $\checkmark$ & & \\
\hline 4 & Lal and Staelin (1986) & $\checkmark$ & & $\checkmark$ & & $\checkmark$ & & \\
\hline 5 & Mantrala and Raman (1990) & $\checkmark$ & & $\checkmark$ & & $\checkmark$ & & \\
\hline 6 & Basu and Kalyanaram (1990) & $\checkmark$ & & $\checkmark$ & & $\checkmark$ & & \\
\hline 7 & Lal and Srinivasan (1993) & $\checkmark$ & & $\checkmark$ & & $\checkmark$ & & \\
\hline 8 & Mantrala et al. (1994) & $\checkmark$ & & $\checkmark$ & & $\checkmark$ & & \\
\hline 9 & Raju and Srinivasan (1996) & $\checkmark$ & & $\checkmark$ & & $\checkmark$ & & \\
\hline 10 & Mantrala et al. (1997) & $\checkmark$ & & $\checkmark$ & & $\checkmark$ & & \\
\hline 11 & Joseph and Thevaranjan (1998) & $\checkmark$ & & $\checkmark$ & & $\checkmark$ & & \\
\hline 12 & Kalra et al. (2003) & $\checkmark$ & & $\checkmark$ & & $\checkmark$ & & \\
\hline 13 & Godes (2003) & $\checkmark$ & & $\checkmark$ & & $\checkmark$ & & \\
\hline 14 & Murthy and Mantrala (2005) & $\checkmark$ & & $\checkmark$ & & $\checkmark$ & & \\
\hline 15 & Misra et al. (2005) & $\checkmark$ & & $\checkmark$ & & $\checkmark$ & & \\
\hline 16 & Misra and Nair (2011) & $\checkmark$ & & $\checkmark$ & & $\checkmark$ & & \\
\hline 17 & Jain (2012) & $\checkmark$ & & $\checkmark$ & & $\checkmark$ & & \\
\hline 18 & Dai and Jerath (2013) & $\checkmark$ & & $\checkmark$ & & $\checkmark$ & & \\
\hline 19 & Syam et al. (2013) & $\checkmark$ & & $\checkmark$ & & $\checkmark$ & & \\
\hline 20 & Yang et al. (2013) & $\checkmark$ & & $\checkmark$ & & $\checkmark$ & & \\
\hline 21 & Rubel and Prasad (2015) & $\checkmark$ & & $\checkmark$ & & $\checkmark$ & & \\
\hline 22 & Daljord et al. (2016) & $\checkmark$ & & $\checkmark$ & & $\checkmark$ & & \\
\hline 23 & Schöttner (2016) & $\checkmark$ & & $\checkmark$ & & $\checkmark$ & & \\
\hline 24 & This Paper & & $\checkmark$ & $\checkmark$ & $\checkmark$ & $\checkmark$ & & $\checkmark$ \\
\hline
\end{tabular}

However, virtually all of this classic agency-theoretic work on sales compensation plans in Marketing have assumed that agents' selling effectiveness values are constant and focused on stimulating the levels and allocations of selling efforts. One notable exception is a paper by Godes (2003) that allows a rep to act to acquire high or low selling skills based on the consumers' or products' complexity.

Consequently, the traditional perspective has three limitations. First, it neglects 
the fact that a sales agent can enhance performance by increasing the quality as well as quantity of selling effort. Second, the sales process is assumed to be one stage. The classic literature has considered that sales are realized with constant selling effectiveness as soon as selling effort is applied when in fact the selling process involves at least two stages that clearly call for different kinds of thinking efforts in addition to selling effort, specifically, 'qualifying and targeting' customers followed by the 'personal selling' phase. Effectiveness of sales calls made by an agent on customers depends on the thought (or 'thinking effort') the agent puts into qualifying and choosing those customer targets as well as presenting her/his offering to those customers (Szymanski 1988). Lastly, the previous literature is limited to considering only sales output-based compensation plans and disregards MD incentive plans that include both sales-based and activity-based incentives, often seen in practice.

On the other hand, the behavioral sales management literature contains empirical evidence in support of the notion of adaptable selling effectiveness especially those papers focused on the quality of work (i.e., working smart) rather than the quantity of work (i.e., working hard). Sujan et al. (1994) define working smart as "the engagement in activities that serve to develop knowledge of sales situations and utilize this knowledge in selling behavior." Sujan et al. (1994) then showed that a learning goal orientation motivates higher selling effectiveness, working smarter as well as harder, but a performance orientation motivates only working harder. Further, Rapp et al. (2006), Fang et al. (2008), Rapp et al. (2008), and Holmes and Srivastava (2002) have shown that working smart implies better sales planning and adaptive selling in contrast to simply working hard (i.e., making more sales calls.) Hence, improving selling effectiveness implies increasing work quality, and my proposed model allows this quality to vary with reps' choices of thinking effort levels.

Furthermore, Anderson and Oliver (1987) and Challagalla and Shervani (1996) have shown that more behavior-based control helps to intrinsically motivate reps to 
improve selling effectiveness while simple outcome-based control decreases the motivation for selling effectiveness improvement. Also, behavior-based compensation plans lead reps to exercise greater sales expense control, plan and make better sales presentations (Oliver and Anderson 1994, Piercy et al. 1998), and reduce biased perceptions of customer relationship quality (Mullins et al. 2014). Other studies have shown that outcome-based compensation plans too can motivate reps to increase selling effectiveness, (e.g., Cravens et al. (1993), Piercy et al. (2004), Ahearne et al. (2010b), Hohenberg and Homburg (2016)). The critical point, however, is that all these studies indicate that selling effectiveness is adaptable and appropriate incentives can drive its improvement.

In this paper, therefore, I make a major departure from the past agency-theoretic literature by allowing selling effectiveness (the coefficient of selling effort, i.e., sales output per unit selling effort), to be (1) adaptable, (2) derived from thinking efforts in two primary stages of the selling process ('qualifying and targeting' stage and 'sales presentation' stage); and (3) affected by how the incentive plan induces the thinking efforts. Specifically, I conceptualize that higher or lower selling effectiveness is determined by the amount and type of thought that salespeople put into the two basic stages of the selling process: 'qualifying' phase and 'selling presentation and closing' phase. Further, there are cognitive costs associated with these thinking efforts, similar to the psychic cost or disutility associated with selling effort. The assumption of costs associated with thinking effort has ample precedence in prior literature (e.g., Simon and Newell 1971, Shugan 1980, Johnson and Payne 1985, Hauser and Wernerfelt 1990). The proposed 'extended' agency-theoretic model is developed in the next section. 


\section{Extended Principal-Agent Model}

I consider a B2B firm with a heterogeneous sales force of size $\mathrm{J}$ with each salesperson or agent $\mathrm{j}, \mathrm{j}=1, \ldots, \mathrm{J}$ assigned to an independent sales territory. I make the following assumptions about each salesperson $\mathrm{j}$ and the firm (the Principal) in developing my model for assessing different forms of compensation plans for this sales force. The first seven of these assumptions (A1)-(A7) below are the standard ones made in previous agency-theoretic sales force compensation models (e.g., Basu et al. 1985, Lal and Srinivasan 1993). The next four, (A8) - (A11) are non-standard and new. Table 2 summarizes notation used in the model development.

Table 2: List of Symbols

\begin{tabular}{ll}
\hline \hline Symbols & Parameters \\
\hline $\mathrm{j}$ & Number of salespeople, $1, \ldots, \mathrm{J}$ \\
$\mathrm{i}$ & Type of stages, 1 means prepping and 2 means selling stage \\
$s_{j}$ & Sales of salesperson $\mathrm{j}$ \\
$k_{j}$ & Targeted activity achievement level of salesperson $\mathrm{j}$ \\
$\alpha_{j}$ & Carryover sales in territory of salesperson $\mathrm{j}$ \\
$W_{j}$ & Compensation earned by salesperson $\mathrm{j}$ \\
$w_{0 j}$ & Fixed salary of salesperson $\mathrm{j}$ \\
$w_{1 j}$ & Commission rate on sales of salesperson $\mathrm{j}$ \\
$w_{2 j}$ & Commission rate on activity of salesperson $\mathrm{j}$ \\
$\mu_{j}$ & Risk-aversion of the salesperson $\mathrm{j}$ \\
$x_{j}$ & Selling effort by salesperson $\mathrm{j}$ \\
$e_{1 j}$ & Thinking effort in prepping stage by salesperson $\mathrm{j}$ \\
$e_{2 j}$ & Thinking effort in selling stage by salesperson $\mathrm{j}$ \\
$\beta_{j}$ & Selling effectiveness by salesperson $\mathrm{j}$ \\
$\delta_{j}$ & Basic ability of salesperson $\mathrm{j}$ \\
$u_{j}$ & Productivity of thinking effort of salesperson $\mathrm{j}$ in prepping stage \\
$\phi_{j}$ & Productivity of thinking effort of salesperson $\mathrm{j}$ in selling stage \\
$\gamma_{1 j}$ & Productivity of effort of salesperson $\mathrm{j}$ for customer requested demos (prepping stage effort) \\
$\gamma_{2 j}$ & Productivity of effort of salesperson $\mathrm{j}$ for customer requested demos (selling stage effort) \\
$c_{j}$ & Salesperson j's cost associated with selling effort \\
$c_{1 j}$ & Salesperson j's cost associated with thinking effort in prepping stage \\
$c_{2 j}$ & Salesperson j's cost associated with thinking effort in selling stage \\
$U_{j}$ & Net utility of salesperson $\mathrm{j}$ \\
$\bar{F}_{j}$ & Reservation wage of salesperson $\mathrm{j}$ \\
$m$ & gross margin \\
$\epsilon_{j}$ & Market uncertainty of salesperson $\mathrm{j}$ \\
$\pi_{j}$ & Net profits of salesperson $\mathrm{j}$ \\
$\sigma_{j}^{2}$ & Variance of market uncertainty of salesperson $\mathrm{j}$ \\
\hline \hline
\end{tabular}

A1) Sales agent j's sales response-effort function is linear in effort with normally distributed random disturbances, i.e., $\hat{S}_{j}=\alpha_{j}+\beta_{j} x_{j}+\varepsilon_{j}, \varepsilon_{j} \sim \mathcal{N}\left(0, \sigma^{2}\right)$ where $\alpha_{j}$ denotes base level of sales of her territory due to carryover and other marketing efforts of the firm, $x_{j} \in \mathbb{Z}^{+}$denotes selling effort (measured by number of calls or visits to targeted customers), $\beta_{j} \in \mathbb{R}^{+}$is the agent's (adaptable, not fixed) selling effectiveness, and $\varepsilon_{j}$ is the random disturbance term, normally distributed with zero mean and variance $\sigma^{2}$. These assumptions with regard to sales response functional 
form and uncertainty distribution are standard and have been justified by Holmstrom and Milgrom (1987), Lal and Srinivasan (1993), Misra et al. (2005), Daljord et al. (2016).

A2) A salesperson's utility or satisfaction derived from work is an additively separable function of her/his income and selling effort. More specifically, the sales rep acts to maximize expected 'net utility', i.e., expected utility of income less disutility associated with efforts involved to earn that income. Mathematically, let $u_{j}\left(w_{j}\right)$ denote the $j$ th rep's utility function for income $w_{j}$ and $c_{j}\left(x_{j}\right)$ denote the cost or disutility associated with her/his selling efforts $x_{j}$. Then the rep's 'net utility' is represented as $u\left(w_{j}\right)-c\left(x_{j}\right)$.

A3) The sales rep's utility of income is concave in income, implying the rep is risk averse, i.e., $u(w) \geqq 0, u^{\prime}(w)>0, u^{\prime \prime}(w)<0$. More specifically, I assume salespeople's utility of income can be specified as a constant absolute risk aversion function, i.e., $u(w)=-\exp \left(-\mu_{j}(w(s))\right)$ where the absolute risk aversion parameter is positive $(\mu>0)$.

A4) The sales rep's disutility is an increasing function of selling effort, i.e., $c(x) \geqq$ $0, c^{\prime}(x)>0, c^{\prime \prime}(x)>0$.

A5) The firm's marginal cost of production of the product or service and gross margin per unit are constant.

A6) The firm is risk neutral and has the objective of maximizing its expected profits $E[\pi]$.

A7) The sales rep's and the firm's information about the form and parameters of the sales rep's sales response function and utility function is symmetric.

Next are my non-standard model assumptions:

A8) I assume that the selling process to a customer has two basic stages of "prepping" and "selling". That is, I collapse to two the multiple stages of the classic selling 
process (e.g., Long et al. 2007) - prospecting, qualifying, pre-approach, approach, presenting and demonstrating, handling objections, closing, and follow-up. Specifically, the prepping stage includes prospecting, qualifying and pre-approach planning to determine whether and what for to target a prospect; while the selling stage includes the remaining stages of the selling process.

A9) The selling effectiveness $\beta_{j}$ of rep $j$, is a function of the amount of "thinking efforts" the rep puts into the prepping and selling stages of the sales process. i.e., $\beta_{j}=\delta_{j}+u_{j} e_{1 j}+\phi_{j} e_{2 j}$ where $\delta_{j}\left(\delta_{j} \in \mathbb{R}^{+}\right)$denotes a constant basic selling ability level of the rep, derived from her/his qualifications, prior experience, sales training; $e_{1 j}\left(e_{1 j} \in \mathbb{R}^{+}\right)$denotes thinking effort in prepping stage, $u_{j}\left(u_{j} \in \mathbb{R}^{+}\right.$, and $0<u_{j} \leq$ 1) denotes the marginal impact of this first stage thinking effort, $e_{2 j}\left(e_{1 j} \in \mathbb{R}^{+}\right)$ represents thinking effort in selling stage, and $\phi_{j}\left(\phi_{j} \in \mathbb{R}^{+}\right.$, and $\left.0<\phi_{j} \leq 1\right)$ denotes the marginal impact of this second stage thinking effort.

Thus, selling effectiveness depends on basic selling ability but is enhanced by the thinking efforts put in by the rep in the two stages of the selling process.

A10) Sales reps have disutility (cost) associated with the thinking efforts in the two stages. More specifically, I posit the total cost of thinking and selling efforts is an additively separable function of the disutility or psychic cost of thinking efforts in the prepping and selling stages, and basic selling effort as specified below: i.e., $c\left(x, e_{1}, e_{2}\right)=\frac{c_{1 j}\left(e_{1 j} x_{j}\right)^{2}}{2}+\frac{c_{2 j}\left(e_{2 j} x_{j}\right)^{2}}{2}+\frac{c_{j} x_{j}^{2}}{2}$, where $c_{j}>0, c_{1 j}>0$, and $c_{2 j}>0$ are the respective cost parameters. Similar to the standard assumption with respect to the perceived cost of sales calls $(x)$, I assume the costs associated with thinking efforts are increasing (convex) functions of the levels of thinking efforts.

A11) Distinct from the marginal cost of production, the firm incurs 'logistical' costs whenever reps call on sales targets. Let the firm's cost related to a rep's calls (stemming from reimbursement of travel expenses such as transportation, accommodation, meals etc) be denoted as $L(x)=v x$ where $v$ is the average logistical cost of 
customer calls $(x)$. Thus, based on the above assumptions, the firm's profit function is expressed by Equation 3.1:

$$
\pi=S m-w(S)-L(x)
$$

where $m$ the constant gross margin (hereafter assumed to be 1 for expositional simplicity) and $w(S)$ is the sales-dependent compensation payout for the salesperson. Note that in the traditional principal-agent model, the sales call costs are not included in the firm's profit function but in practice, it is quite common for firms to reimburse the agent's travel costs. This implies the firm pays a price for calls made without much thought by the reps. That is, the firm incurs higher costs when reps work hard (make more sales calls) without being smart about whom they call on (e.g., Oliver and Anderson 1994). Next, [Figure 1] depicts the sequence of moves in the firm-salesperson interaction.

\begin{tabular}{ccc} 
Stage 1 & Stage 2 & Stage 3 \\
\hline $\begin{array}{c}\text { Firm announces } \\
\text { compensation plan }\end{array}$ & $\begin{array}{c}\text { Salespeople allocate efforts } \\
\text { but the firm does not observe } \\
\text { both thinking and selling } \\
\text { actions }\end{array}$ & $\begin{array}{c}\text { Firm observes their profit } \\
\text { and pays the compensation }\end{array}$ \\
$\begin{array}{c}\text { and } \\
\text { and }\end{array}$ &
\end{tabular}

Figure 1: Sequence of move between firms and salespeople

\section{Extended PA Model for Salesperson Compensation with Adapt- able Selling Effectiveness}

As already noted, I specify sales agent j's sales response function with adaptable selling effectiveness as expressed in Equation 3.2;

$$
\hat{S}_{j}=\alpha_{j}+\left(\delta_{j}+u_{j} e_{1 j}+\phi_{j} e_{2 j}\right) x_{j}+\varepsilon_{s j}, \quad \varepsilon_{s j} \sim \mathcal{N}\left(0, \sigma_{s j}^{2}\right)
$$


In deriving the optimal compensation plan structure, I assume the firm is the Stackelberg leader in its interaction with the $j$ th agent (as shown in Figure 1) and sets the plan that maximizes its expected profit subject to two constraints as shown in model M1 below: the rep's Individual Rationality (IR) and Incentive Compatibility (IC) constraints.

(M1)

$$
\max _{w_{1 j} \in \mathbb{N}} \mathrm{E}[\pi]=\int_{\underline{s}}^{\bar{s}}\left[S_{j}-W\left(S_{j}\right)-L_{j}\right] f\left(s_{j}\right) d s_{j}
$$

s.t:

$$
\begin{gathered}
(I R) \mathrm{E}\left[U_{j}\left(W_{j}-c\left(x_{j}, e_{1 j}, e_{2 j}\right)\right)\right] \geq \bar{F}_{j}, \forall j=1, \ldots, J \\
(I C) x_{j}\left(W_{j}\right), e_{1 j}\left(W_{j}\right), e_{2 j}\left(W_{j}\right) \in \underset{x_{j} \in \mathbb{N}, e_{1 j} \in \mathbb{N}, e_{2 j} \in \mathbb{N}}{\arg \max } \mathrm{E}\left[U\left(W_{j}-c\left(x_{j}, e_{1 j}, e_{2 j}\right)\right)\right], \forall j=1, \ldots, J
\end{gathered}
$$

where $\mathrm{J}$ is the number of sales reps, $\mathbb{N}$ is the natural number set, $(\bar{s}, \underline{s})$ are the upper and lower bound for amount of sales and $\bar{F}_{j}$ is the rep's reservation utility.

\section{Alternative Compensation Plan Scenarios with Adaptable Selling Effectiveness}

To answer my research questions, I solve the above principal-agent model for the optimal decisions of heterogeneous reps and the firm under four alternative compensation plan scenarios: The 'standard' linear salary plus pure sales-based commission plan whose parameters are either uniform or customized across the sales force; versus a linear form of 'multidimensional' plan (salary plus sales- plus activity-based commission plan) with either uniform or customized parameters across the sales force (see Figure 2). The optimality of uniform or 'common' plans relative to customized plans for a heterogeneous sales force is an important issue given the widespread use of uniform plans in practice. In theory, customized plans should yield superior returns for the 
firm but uniform plans are often preferred in practice because they appear equitable and are less costly to implement and administer (e.g., Mantrala et al. 1994, Misra and Nair 2011). Recently, Daljord et al. (2016) focused on this issue and showed that a firm's payoff under a uniform plan comes very close to that under a fully customized plan when the firm can optimize both sales force compensation and sales force composition. Following this interesting insight, I ask here if uniform plans can be almost as good as customized plans even when the composition is fixed, but salespeople have adaptable selling effectiveness? Also, how different are reps' and the firm's payoffs under uniform and customized multidimensional plans?

\begin{tabular}{c|cc}
\hline Compensation & Customized & Uniform \\
\hline \multirow{4}{*}{ Standard } & 4.1 Standard- & 4.3 Standard- \\
& Customized (SC) & Uniform (SU) \\
& & \\
Multidimensional & 4.2 Multidimensional- & 4.4 Multidimensional- \\
& Customized (MD-C) & Uniform (MD-U) \\
\hline
\end{tabular}

Figure 2: Types of Compensation Plans

In the following sections 4.1-4.4, I detail the compensation plan specification, model M1 solution and comparative statics results in each of the four scenarios 4.1 Standard-Customized, 4.2 Multidimensional-Customized, 4.3 Standard-Uniform, and 4.4 Multidimensional-Uniform plans (see Web Appendix 7.2). Table 3 summarizes the firm's and sales reps' optimal choices under the four different compensation plan scenarios. Notably, although I have many parameters, I obtain a closed form solution for optimal incentives and efforts in all four scenarios. I now proceed to a detailed description and discussion of each of these scenario analyses and results. 
Table 3: Four Principal-Heterogeneous Agent Model Solutions

\begin{tabular}{|c|c|c|c|c|}
\hline & $\begin{array}{l}\text { Standard } \\
\text { Custom }\end{array}$ & $\begin{array}{l}\text { Standard } \\
\text { Uniform } \\
\end{array}$ & $\begin{array}{l}\text { Multidim } \\
\text { Custom }\end{array}$ & $\begin{array}{l}\begin{array}{l}\text { Multidim } \\
\text { Uniform }\end{array} \\
\text { Unt }\end{array}$ \\
\hline$x_{j}$ & $x_{s c, j}^{*}=\frac{w_{s c, 1 j}^{*} \delta_{j}}{c_{j}}$ & $x_{s u, j}^{*}=\frac{\bar{w}_{s u, 1}^{*} \delta_{j}}{c_{j}}$ & $x_{m c, j}^{*}=\frac{w_{m c, 1 j}^{*} \delta_{j}}{c_{j}}$ & $x_{m u, j}^{*}=\frac{w_{m u, 1}^{*} \delta_{j}}{c_{j}}$ \\
\hline$e_{1}$ & $e_{s c, 1 j}^{*}=\frac{u_{j} c_{j}}{c_{1 j} \delta_{j}}$ & $e_{s u, 1 j}^{*}=\frac{u_{j} c_{j}}{c_{1 j} \delta_{j}}$ & $e_{m c, 1 j}^{*}=\frac{c_{j} u_{j}}{c_{1 j} \delta_{j}}+\frac{c_{j} \gamma_{1 j} j}{c_{1 j} \delta_{j} j} \frac{w_{m c, 2 j}^{*}}{w_{m c 1 j}^{*}}$ & $e_{m u, 1 j}^{*}=\frac{c_{j} u_{j}}{c_{1 j} \delta_{j}}+\frac{c_{j} \gamma_{11}}{c_{1 j} \delta_{j}} \frac{\bar{w}_{m u, 2}^{*}}{\bar{w}_{m u, 2}^{*}}$ \\
\hline$e_{2}$ & $e_{s c, 2 j}^{*}=\frac{\phi_{j} c_{j}}{c_{2 j} \delta_{j}}$ & $e_{s u, 2 j}^{*}=\frac{\phi_{j} c_{j}}{c_{2 j} \delta_{j}}$ & $e_{m c, 2 j}^{*}=\frac{c_{j} \phi_{j}}{c_{2 j} \delta_{j}}+\frac{c_{j} \gamma_{2 j}}{c_{2 j} \delta_{j}} \frac{w_{m c, 2 j}^{*}}{w_{m c, 1 j}^{*}}$ & $e_{m u, 2 j}^{*}=\frac{c_{j} \phi_{j}}{c_{2 j} \delta_{j}}+\frac{c_{j} \gamma_{2 j}}{c_{2 j} \delta_{j}} \frac{\bar{w}_{m u, 2}^{*}}{\bar{w}_{m u, 1}^{*}}$ \\
\hline$w_{1}$ & $w_{s c, 1 j}^{*}=\frac{y_{j}}{2 a_{j}}$ & $\bar{w}_{s u, 1}^{*}=\frac{\sum_{j=1}^{J} \lambda_{j}}{2 \sum_{j=1}^{J} \rho_{j}}$ & $w_{m c, 1 j}^{*}=\frac{\alpha_{j}+\delta_{j}\left(\delta_{j}-v_{j}\right) b_{j}}{2\left(\alpha_{j}+h_{j} b_{j}\right)}$ & $\bar{w}_{m u, 1}^{*}=\frac{\sum_{j=1}^{J} \lambda_{j} \sum_{j=1}^{J} \eta_{j}-\left(\sum_{j=1}^{J} \theta_{j}\right)^{2}}{2\left(\sum_{j=1}^{J} \rho_{j} \sum_{j=1}^{J} \eta_{j}-\left(\sum_{j=1}^{J} \theta_{j}\right)^{2}\right)}$ \\
\hline$w_{2}$ & & & $w_{m c, 2 j}^{*}=\frac{k_{j}}{b_{j}}\left(\frac{1}{2}-w_{1}^{*}\right)$ & $\bar{w}_{m u, 2}^{*}=\frac{\sum_{j=1}^{J} \theta_{j}}{\sum_{j=1}^{J} \eta_{j}}\left(\frac{1}{2}-\bar{w}_{m u, 1}^{*}\right)$ \\
\hline
\end{tabular}

note: $y_{j}=\delta_{j} c_{1 j} c_{2 j}\left(\delta_{j}-v\right)+c_{j}\left(c_{j 1} \phi_{j}^{2}+c_{2 j} u_{j}^{2}\right), \quad a_{j}=\left(c_{j} c_{1 j} c_{2 j} \mu_{j} \sigma_{S j}^{2}+c_{1 j} c_{2 j} \delta_{j}^{2}+c_{j}\left(c_{1 j} \phi_{j}^{2}+c_{2 j} u_{j}^{2}\right)\right)$, $\alpha_{j}=c_{j}\left[\left(\phi_{j} \gamma_{1 j}-u_{j} \gamma_{2 j}\right)^{2}+\left(c_{1 j} \phi_{j}^{2}+c_{2 j} u_{j}^{2}\right) \mu_{j} \sigma_{K j}^{2}\right], \quad b_{j}=\gamma_{1 j}^{2} c_{2 j}+\gamma_{2 j}^{2} c_{1 j}+\mu_{j} \sigma_{K j}^{2} c_{1 j} c_{2 j}, \quad h_{j}=$ $\delta_{j}^{2}+\mu_{j} \sigma_{S j}^{2} c_{j}$,

$k_{j}=\gamma_{2 j} c_{1 j} \phi_{j}+\gamma_{1 j} c_{2 j} u_{j}, \quad \lambda_{j}=\frac{\left(\delta_{j}-v\right) \delta_{j} c_{1 j} c_{2 j}+c_{j}\left(c_{2 j} u_{j}^{2}+c_{1 j} \phi_{j}^{2}\right)}{c_{j} c_{1 j} c_{2 j}}$,

$\eta_{j}=\frac{\left(c_{1 j} \gamma_{2 j}^{2}+c_{2 j} \gamma_{1 j}^{2}\right)}{c_{1 j} c_{2 j}}+\mu_{j} \sigma_{K j}^{2}, \quad \theta_{j}=\frac{\left(\gamma_{1 j} c_{2 j} u_{j}+\gamma_{j} c_{1 j} \phi_{j}\right)}{c_{1 j} c_{2 j}}$,

$\rho_{j}=\frac{c_{j}\left(c_{2 j} u_{j}^{2}+c_{1 j} \phi_{j}^{2}\right)+\delta_{j}^{2} c_{1 j} c_{2 j}+c_{j} c_{1 j} c_{2 j} \mu_{j} \sigma_{S j}^{2}}{c_{j} c_{1 j} c_{2 j}}$

\subsection{Benchmark: Standard-Customized plan}

In this benchmark scenario, I assume the firm employs a standard form of compensation plan, i.e., a salary plus straight commission plan, to motivate its sales force. Further, I suppose the firm sets the optimal customized plan parameters for each of its heterogeneous salespeople. My objective is to derive the optimal plan parameters and outcomes for each salesperson and the firm when selling effectiveness is adaptable and compare these results with those obtained under the standard compensation plan and standard assumption of fixed selling effectiveness. Thereby, I provide some new insights into how the realistic assumption of adaptable selling effectiveness alters the traditional results that have appeared in the literature so far (Basu et al. 1985, Basu and Kalyanaram 1990, Joseph and Thevaranjan 1998, Lal and Srinivasan 1993). I remind readers that in my model reps choose thinking efforts as well as selling effort to maximize their expected utility unlike the traditional principal-agent models in which agents choose only selling effort.

I begin the analysis with the specification of the standard-customized plan $\left(W_{s c, j}\right)$ 
under consideration for rep $\mathrm{j}, \mathrm{j}=1, \ldots \mathrm{J}$ :

$$
W_{s c, j}=w_{s c, 0 j}+w_{s c, 1 j} S_{j}
$$

where $w_{s c, 0 j}$ is the rep's basic salary, $w_{s c, 1 j}$ is the customized sales commission under the standard-customized plan. Upon solving Model M1 after incorporating Equation 4.1 in it, I obtain the sales rep's optimal efforts $\left(x_{s c, j}^{*}\right)$ and firm's optimal commission rates $\left(w_{s c, 1 j}^{*}\right)$ shown in the first column of the Table 3. Based on the these results I offer insights into the derived solution to the sales reps' problem in proposition 1.

Proposition 1. All else equal,

(a) An increase in sales commission rate $w_{1 j}$, will increase rep j's selling effort but not impact his/ her thinking efforts nor selling effectiveness.

(b) Total efforts allocation:

b-1) If $0<\frac{\delta_{j}}{c_{j}}<1$, sales rep $j$ will decrease his/ her selling effort and increase both thinking efforts evenly.

b-2) If $\frac{\delta_{j}}{c_{j}}=1$, sales rep $j$ will choose his/ her three efforts as follows:

$$
x=w_{1 j}, \quad e_{1 j}=\frac{u_{j}}{c_{1 j}}, \quad e_{2 j}=\frac{\phi_{j}}{c_{2 j}}
$$

b-3) If $1<\frac{\delta_{j}}{c_{j}}$, sales rep $j$ will increase his selling effort and decrease both thinking efforts evenly.

Proof: See appendix 6.1.1.

According to Proposition 1(a), interestingly, the sales rep's thinking effort choices are not affected by sales commission rate under the standard compensation plan. Since the firm gives incentive on just the sales outcome, one might think, at first glance, that sales reps can choose to increase either or both thinking and selling efforts 
to raise their sales. However, the model solution indicates that optimal thinking efforts are unaffected by the commission rate $\left(e_{1 j}^{*} \neq f\left(w_{s c, 1 j}\right)\right)$. This result, formally shown for the first time, is consistent with previous empirical findings that purely outcome-based compensation does not improve selling effectiveness (Weitz et al. 1986, Spiro and Weitz 1990, Piercy et al. 2004).

More interestingly, Proposition 1(b) implies that a rep will decrease (increase) selling effort and increase (decrease) thinking efforts when base level of selling ability is smaller (larger) relative to selling effort cost. This implies the base level of ability and selling effort cost impact optimal allocations of both thinking and selling efforts. Qualitatively, a higher disutility or cost of selling effort suggests customers who are more well-informed and "harder nuts to crack." To cope with this challenge, the rational salesperson realizes s/he must augment her/his basic ability with more deliberative thinking efforts rather than simply put in more selling effort.

Additionally, the relative magnitudes of each thinking effort's effectiveness to cost ratio determines which dominates as would be expected. Salespeople will strategically choose their thinking effort to enhance their selling effectiveness and maximize their utility and satisfaction. If the rep $\mathrm{j}$ deems that the disutility from putting more thinking effort into 'prepping' is less than that of putting thinking effort into selling $\left(\frac{u_{j}}{c_{1 j}}>\frac{\phi_{j}}{c_{2 j}}\right)$, then the rep j will choose to increase prepping-related thinking effort more than selling thinking effort.

\section{Comparative Statics Results}

I now examine how the endogenous outputs of my model solution are impacted by changes in the exogenous parameters of the model such as uncertainty, risk aversion, marginal cost, minimum utility requirement, base sales level and disutility parameters associated with selling and thinking efforts. The results are reported in Table 4. Most of these results are consistent with those of Lal and Srinivasan (1993) (p. 785) derived 
from the traditional model that assumes fixed selling effectiveness with selling effort as the sole choice variable for the rep. The derivation of all comparative static results are provided in the Appendix 7.2.

Table 4: Comparative Statics in Standard-Customized commission

\begin{tabular}{|c|c|c|c|c|c|c|c|c|c|c|}
\hline Effect of & $\pi$ & $x$ & $e_{1}$ & $e_{2}$ & $\beta$ & $w_{1}$ & $w_{2}$ & $E[w]$ & $\frac{w_{0}}{E[w]}$ & $\frac{w_{2}}{w_{1}}$ \\
\hline Uncertainty $\left(\Uparrow \sigma_{s}^{2}\right)$ & - & - & 0 & 0 & 0 & - & & - & + & \\
\hline Risk Aversion $(\Uparrow \mu)$ & - & - & 0 & 0 & 0 & - & & - & + & \\
\hline Cost of Selling Effort $(\Uparrow c)$ & - & - & + & + & + & -+ & & - & + & \\
\hline Cost of Thinking Effort1 $\left(\Uparrow c_{1}\right)$ & - & - & - & 0 & - & - & & - & + & \\
\hline Cost of Thinking Effort2 $\left(\Uparrow c_{2}\right)$ & - & - & 0 & - & - & - & & - & - & \\
\hline Basic Ability $(\Uparrow \delta)$ & -+ & + & - & - & -+ & -+ & & -+ & -+ & \\
\hline Thinking 1 Effectiveness $(\Uparrow u)$ & + & + & + & 0 & + & + & & + & - & \\
\hline Thinking 2 Effectiveness $(\Uparrow \phi)$ & + & + & 0 & + & + & + & & + & - & \\
\hline Cost of Activity $(\Uparrow \nu)$ & -+ & - & 0 & 0 & 0 & - & & -+ & -+ & \\
\hline Opportunity Cost $(\bar{F})$ & - & 0 & 0 & 0 & 0 & 0 & & + & + & \\
\hline
\end{tabular}

1. + goes up, - goes down, 0 no change.

2. Comparative statics results corresponding to the rows for $c, c_{1}, c_{2}, u, \phi$, and $\nu$ are unavailable in Lar and Srinivasan (1993).

For example, I find from my model that uncertainty $\left(\sigma_{s}^{2}\right)$ and risk aversion $(\mu)$ have negative relationships with optimal selling effort $(x)$, optimal incentive rate $\left(w_{1}\right)$, reps' expected income $(E[w])$, and firm's profit $(\pi)$. Accordingly, the ratio of salary to expected total income $\left(\frac{w_{0}}{E[w]}\right)$ increases when the uncertainty $\left(\sigma_{s}^{2}\right)$ or risk aversion $(\mu)$ increases. Also, the fixed basic ability parameter $(\delta)$ is equal to the fixed sales effectiveness parameter (k) in Lal and Srinivasan (1993). Then, an increase in base selling ability positively affects the optimal profit, selling effort, and expected income. Consequently, the ratio of salary to the expected total income $\left(\frac{w_{0}}{E[w]}\right)$ decreases when the base ability increases. Last, the opportunity $\operatorname{cost}(\bar{F})$ is equivalent to the minimum expected utility (m) in Lal and Srinivasan (1993). Similar to their findings, an increase in opportunity cost does not affect selling effort $(x)$ and optimal incentive $\left(w_{1}\right)$, positively affects expected income $(E[w])$ and the ratio of salary to total income $\left(\frac{w_{0}}{E[w]}\right)$, and negatively affects optimal profit $(\pi)$.

However, interestingly, in the new model with adaptable selling effectiveness, the 
uncertainty $\left(\sigma_{s}^{2}\right)$ and risk aversion $(\mu)$ parameters do not affect the optimal choices of thinking efforts $\left(e_{1}, e_{2}\right)$ or overall selling effectiveness $(\beta)$. It makes sense that agents do not vary their thinking efforts with changes in market uncertainty or risk aversion. Rather, a salesperson's optimal thinking effort levels respond to changes in effort costs $\left(c, c_{1}, c_{2}\right)$, thinking effort effectiveness $(u, \phi)$, and base ability $(\delta)$. For instance, when the cost of selling effort $(c)$ increases, reps will increase thinking efforts $\left(e_{1}, e_{2}\right)$. As a result of increasing thinking efforts, the selling effectiveness $(\beta)$ will also increase. Intuitively, an increase in the cost of selling effort implies a more difficult selling environment where increased selling effectiveness pays off more than increased selling effort, and the disutility of selling effort increases relative to the disutility of thinking efforts. Consequently, the rep shifts from more selling effort to more thinking efforts that enhance selling effectiveness.

Another interesting insight is that while a change in $(\delta)$ has an ambiguous impact on the firm's expected profit, the latter are unambiguously affected by a change in thinking effort effectiveness $(u, \phi)$ and the costs of thinking $\left(c_{1}, c_{2}\right)$. Specifically, the firm's expected profit increases as the two thinking effort coefficients increase because of the resultant higher selling effectiveness. The firm's expected profit also increases when the costs of thinking efforts $\left(c_{1}, c_{2}\right)$ increase because the sales agent then increases his/her selling effort. However, the base selling ability $(\delta)$ impacts the firm's profit $(\pi)$ in a nonlinear fashion because as it increases, the rep puts in less thinking effort. The net effect then can be a decline of selling effectiveness which in turn can lower the rep's performance and the firms' profit. However, if the effectiveness of the rep's thinking efforts in the two selling stages is high, s/he may increase thinking as well as selling efforts, thereby boosting the firm's expected profit. Consequently, firms need to emphasize both thinking and selling abilities when recruiting new salespeople.

Table 4 indicates how a salesperson's optimal selling and thinking efforts vary with 
her/his base ability level. Consistent with previous results, my analysis finds that the rep's optimal selling effort $\left(x^{*} \Uparrow\right)$ increases with an increase in base selling ability $\left(\delta_{j} \Uparrow\right)$. However, my analysis also provides some new insights into the relationship between rep's base selling ability and her/his selling effectiveness as summarized in following Proposition 2.

Proposition 2. All else equal, in a model with adaptable selling effectiveness, (a) When $\delta_{j}<\sqrt{c_{j}\left(\frac{u_{j}^{2}}{c_{1 j}}+\frac{\phi_{j}^{2}}{c_{2 j}}\right)}$, sales rep's base ability $\left(\delta_{j}\right)$ and selling effectiveness $\left(\beta^{*}\right)$ have negative (-) relationship.

(b) When $\delta_{j}>\sqrt{c_{j}\left(\frac{u_{j}^{2}}{c_{1 j}}+\frac{\phi_{j}^{2}}{c_{2 j}}\right)}$, sales rep's base ability and selling effectiveness have positive $(+)$ relationship.

Proof: See appendix 6.1.2.

In other words, within the context of my extended PA model, when the rep behaves optimally, selling effectiveness has a u-shape relationship with the rep's base ability $\left(\delta_{j}\right)$. This implies that lower base selling ability salespeople will compensate with increased thinking efforts $\left(e_{j}\right)$ to enhance their selling effectiveness $\left(\beta_{\text {new }}=f\left(e_{j}\right) \Uparrow\right)$ and resulting outcomes. However, the thinking effort levels decline as the rep's base selling ability rises to the critical value $\sqrt{c_{j}\left(\frac{u_{j}^{2}}{c_{1 j}}+\frac{\phi_{j}^{2}}{c_{2 j}}\right)}$, and the combination of low levels of thinking efforts and relatively low selling effort produces the smallest sales outcome at this point. Thus sales reps can be broadly categorized into three types; high base sales ability with high selling effort $(\delta \Uparrow, x \Uparrow, e \Downarrow)$, low base sales ability with high thinking effort $(\delta \Downarrow, x \Downarrow, e \Uparrow)$, and intermediate base sales ability with lower levels of thinking efforts as well as selling effort [Figure 3].

In contrast, the traditional model dose not allow for a salesperson to change her/his selling effectiveness. Equation 4.2 expresses the optimal choices for the rep and the firm in the traditional model.

$$
x_{t r d, j}^{*}=\frac{w_{1 j}^{*} \beta_{j}}{c_{j}}, \quad w_{t r d, 1 j}^{*}=\frac{\beta_{j}^{2}}{2\left(\beta_{j}^{2}+c_{j} \mu_{1 j} \sigma_{j}^{2}\right)} \text { where } \beta_{j}=\delta_{j}
$$




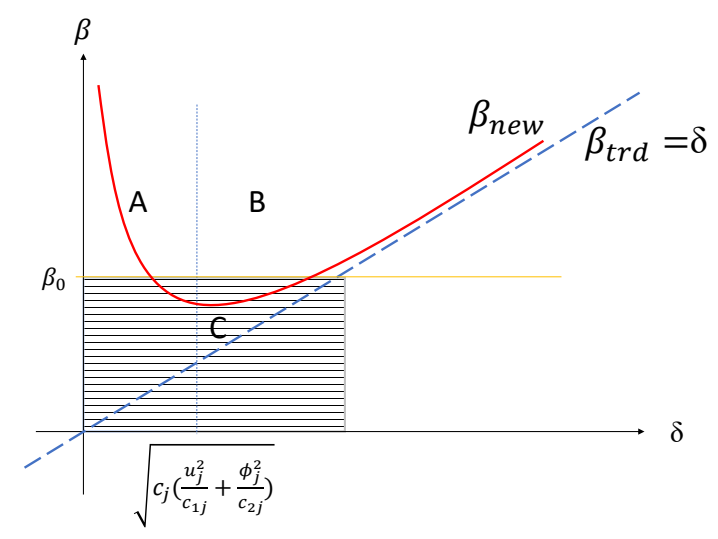

Figure 3: Comparison of Selling Effectiveness in Traditional model $\beta_{t r d}$ and in New model $\beta_{\text {new }}$

$x_{t r d, j}^{*}$ is the sales rep's optimal selling effort and $w_{t r d, 1 j}^{*}$ represents the firm's optimal incentive on sales outcome. Under the traditional model, therefore, optimal selling effort and resulting sales decline as the rep's base selling ability decreases as the model does not allow that a rep can still strive to achieve high overall selling effectiveness and sales with smarter selection of sales targets and messages.

The convex line shows the relationship in the new model with adaptable selling effectiveness.

Proposition 3. All else equal, in a model with adaptable selling effectiveness,

(a) When $\delta_{j}<\tilde{\delta}$, sales rep's base ability and optimal incentive have a negative relationship.

(b) When $\delta_{j} \geq \tilde{\delta}$, rep's base ability and the optimal incentive have a positive relationship. Note: $\tilde{\delta}=-\frac{c_{j} \mu_{j} \sigma_{j}^{2}}{v}+\frac{\sqrt{c_{j} c_{1 j} c_{2 j}\left(c_{j} c_{1 j} c_{2 j} \mu_{j} \sigma_{s j}^{2}+v^{2}\left(c_{1 j} \phi_{j}^{2}+c_{2 j} u_{j}^{2}+\mu_{j} \sigma_{j}^{2}\right)\right)}}{c_{1 j} c_{2 j} v}$

Proof: See appendix 6.1.2.

Proposition 3 implies that the optimal incentive $\left(w_{1 j}^{*}\right)$ and the rep's base ability $\left(\delta_{j}\right)$ have a u-shaped relationship. 


\subsection{Multidimensional-Customized Plan}

Next, I focus on the proposed 'multidimensional' (MD) plan for inducing greater selling effectiveness as well as selling effort. Specifically, this section is concerned with the multidimensional customized (MD-C) plan. The activity metrics that are of interest to us are those that are controllable by both the salesperson and manager, stimulate greater (deliberative) thinking efforts and are 'leading indicators' of sales such as product demos as explained in the Introduction. Inducing a customer request for and giving an appropriate demo during a sales call requires deliberative thinking effort by the salesperson, indicates positive interest in learning more about the product on the part of the customer, and can be a critical step on the way to a successful closure of the call (e.g., Heiman and Muller 1996). However, while standard sales management textbooks and related practitioner blogs note and describe the use of such activity-based bonuses, their formal integration with the regular sales-based incentive plan is never discussed (Schreiner 2018). This is also true of academic sales force incentives research. Therefore, the insights from my comparative analyses of sales force performance under sales-based versus a multidimensional plan structure constitute a significant new contribution to both the academic and practitioner sales management literatures.

More specifically, for illustrative purposes, and without loss of generality, consider the selected activity metric is the number of product demonstrations, ' $\mathrm{K}$ ', made by a salesperson during sales calls in the following analyses. I posit that this number is a function of the number of calls and the average thinking effort levels in the two stages of each call's selling process. That is, to generate more demos, a salesperson needs to put in more deliberative thinking effort in the prepping stage of each call to identify a good target, as well as in the selling stage to induce the target to ask for

a demo. Thus, by tying a compensation component to $\mathrm{K}$, the reps have an incentive to increase their average thinking efforts that improve both their selling effectiveness 
and ultimate sales as well. In contrast, if the activity measurement is just the number of calls made (x), the sales rep can achieve incentives easily by increasing the number of calls without increasing his/her deliberative thinking efforts. Thus, I specify $\mathrm{K}$ as:

$$
K=\left(\gamma_{1} e_{1}+\gamma_{2} e_{2}\right) x+\varepsilon_{k j}, \quad \varepsilon_{k j} \sim \mathcal{N}\left(0, \sigma_{k j}^{2}\right)
$$

where $\gamma_{1}>0$ and $\gamma_{2}>0$, and $\varepsilon_{k j}$ is associated measurement error, arising from random disturbances impacting the number of demonstrations actually given and/or intentional misreporting. To reiterate, given that the ultimate goal is to raise both selling effectiveness and sales, higher performance on the 'activity' rewarded by the proposed MD-C plan must induce greater thinking efforts.

Then, considering a simple additive extension of the simple linear sales-based contract utilized in previous papers (e.g., Daljord et al. 2016, Lal and Srinivasan 1993, Holmstrom and Milgrom 1987) to incorporate the activity-based commission, the multidimensional-customized plan for a sales rep is specified as:

$$
W_{m c, j}=w_{m c, 0 j}+w_{m c, 1 j} S_{j}+w_{m c, 2} K_{j}
$$

where $w_{M C, 0 j}$ is the rep's basic salary, $w_{M C, 1 j}$ is the customized sales incentive, and $w_{M C, 2}$ is the customized activity incentive under MD-C plan. Certainly, modern firms are increasingly using activity-based incentives (e.g., Cloudapps, Hawk Incentives ${ }^{1}$ ).

Lemma 4.1. If a rep puts in zero thinking effort $\left(e_{1}=e_{2}=0\right)$, s/he will receive lower wages than when s/he puts in the optimal thinking efforts $\left(\hat{W}_{m c, j}<W_{m c, j}^{*}\right)$.

In the above lemma, $\hat{W}_{m c, j}$ is the wage when the rep puts in zero thinking efforts and $W_{m c, j}^{*}$ is the wage when the rep puts the optimal thinking efforts. The thinking efforts are drivers of both selling effectiveness and the activity metric K. When a rep

\footnotetext{
${ }^{1}$ https://www.cloudapps.com/effective-sales-incentives/, https://www.hawkincentives.com/insights/moreeffective-sales-incentives
} 
Table 5: Comparative Statics in Multidimensional-Customized commission

\begin{tabular}{l|cccccccccc}
\hline \hline Effect of & $\pi$ & $x$ & $e_{1}$ & $e_{2}$ & $\beta$ & $w_{1}$ & $w_{2}$ & $E[w]$ & $\frac{w_{0}}{E[w]}$ & $\frac{w_{2}}{w_{1}}$ \\
\hline Uncertainty $\left(\Uparrow \sigma_{s}^{2}\right)$ & - & - & + & + & + & - & + & - & + & + \\
Measurement Error $\left(\Uparrow \sigma_{k}^{2}\right)$ & - & - & - & - & - & - & - & - & + & - \\
Risk Aversion $(\Uparrow \mu)$ & -+ & - & + & + & + & - & + & -+ & -+ & + \\
Cost of Selling Effort $(\Uparrow c)$ & -+ & - & + & + & -+ & -+ & -+ & - & + & -+ \\
Cost of Thinking Effort1 $\left(\Uparrow c_{1}\right)$ & - & - & - & + & - & - & + & - & + & + \\
Cost of Thinking Effort2 $\left(\Uparrow c_{2}\right)$ & - & - & + & - & - & - & + & - & + & + \\
Basic Ability $(\Uparrow \delta)$ & -+ & + & - & - & -+ & -+ & -+ & + & - & -+ \\
Thinking 1 Effectiveness $(\Uparrow u)$ & + & + & + & - & + & + & - & + & - & - \\
Thinking 2 Effectiveness $(\Uparrow \phi)$ & + & + & - & + & + & + & - & + & - & - \\
Cost of Activity $(\Uparrow \nu)$ & -+ & - & + & + & + & - & + & -+ & -+ & + \\
Opportunity Cost $(\bar{F})$ & - & 0 & 0 & 0 & 0 & 0 & 0 & + & + & 0 \\
\hline \hline
\end{tabular}

1. + goes up, - goes down, 0 no change.

2. Comparative statics results corresponding to the rows for $\sigma_{k}^{2}, c, c_{1}, c_{2}, u, \phi$, and $\nu$ are unavailable in Lar and Srinivasan (1993).

does not put in the thinking efforts that result in demos to potential clients, her/his selling effectiveness will be reduced and overall sales and, consequently, compensation will also suffer. That is, by not striving for demos, the rep will be indirectly punishing her/himself.

I find two interesting differences in Table 5 that shows the comparative statics results under the MD-C compensation plan. First, the MD-C compensation plan allows firms to adjust incentive strategy in the presence of market uncertainty $\left(\sigma_{s j}^{2}\right)$ and the activity metric 'K's' measurement error $\left(\sigma_{k j}^{2}\right)$. More specifically, the market uncertainty $\left(\sigma_{s j}^{2}\right)$ affects thinking efforts $\left(e_{1 j}, e_{2 j}\right)$ and selling effectiveness $\left(\beta_{j}\right)$ positively. When market uncertainty increases, the optimal incentive on activity $\left(w_{2}\right)$ increases relative to the sales-based incentive $\left(\frac{w_{2}}{w_{1}}\right)$. Since $\frac{w_{2}}{w_{1}}$ influences the thinking efforts under the MD-C plan, the rep's optimal $e_{1 j}, e_{2 j}$ and $\beta_{j}$ increase. That is, the firm can strategically motivate reps to improve selling effectiveness using $w_{2}$ although salespeople want to work less $(x)$ when there is high market uncertainty. On the other hand, the error associated with activity metric ' $\mathrm{K}$ ' $\left(\sigma_{k j}^{2}\right)$ is internally caused by the sales reps or firm's monitoring system. While firms cannot control market uncer- 
tainty, they can reduce the internal measurement error by using improved monitoring technologies. Thus, as the firm reduces this error, the optimal activity incentive it offers $\left(w_{2}\right.$ and $\left.\frac{w_{2}}{w_{1}}\right)$ increases, thereby inducing the rep to put in more thinking and selling efforts.

Under the MD-C plan, risk aversion $\left(\mu_{j}\right)$ positively affects the activity incentive $\left(w_{2}\right)$ and the ratio of the activity to selling incentives $\left(\frac{w_{2}}{w_{1}}\right)$. Increasing activity incentive results in the increase of the sales reps' optimal thinking efforts $\left(e_{1 j}, e_{2 j}\right)$ and selling effectiveness $\left(\beta_{j}\right)$. Further, since the firm's optimal activity incentive $\left(w_{2}\right)$ increases under high cost of activity $(\nu)$, there is a corresponding increase in the rep's optimal thinking efforts $\left(e_{1 j}, e_{2 j}\right)$ and the selling effectiveness $\left(\beta_{j}\right)$. Also, the firm's optimal profit $(\pi)$ may increase with the rep's risk aversion because of the corresponding increase of selling effectiveness $(\beta)$. Without the activity incentive, high risk aversion $(\mu)$ and high cost of activity $(\nu)$ always decrease selling effort and the firm's profit (Table 4). In other words, the advantage of the MD-C plan is that it can actually induce higher selling effectiveness in a poor situation, e.g., one of high cost and high risk aversion.

Proposition 4. Sales rep's Strategy under MD-C Plan

(a) If $w_{1 j}$ increases, the rep $j$ will increase selling effort $\left(x_{j}\right)$ and decrease thinking efforts $\left(e_{1 j}, e_{2 j}\right)$. However, if $w_{2 j}$ increases, the rep will increase thinking effort but not change selling effort.

(b) The rep $j$ will choose his thinking efforts $\left(e_{1 j}, e_{2 j}\right)$ depending on $\frac{w_{2}}{w_{1}}$.

(c) The optimal thinking effort levels under the multidimensional compensation plans are larger than the optimal thinking effort levels under the standard compensation plans.

Proof: See appendix 6.1.5.

Proposition 4(a) implies that a rep's choice of selling efforts only depends on the sales-based incentive $\left(w_{1}\right)$. Since the activity measure $\mathrm{K}$ also includes the selling ef- 
fort $(x)$ in Equation 4.3, there may be a concern of over-payment on selling efforts, i.e., if the commission on activity $\left(w_{2}\right)$ affects selling effort $(x)$, the MD-C plan compensates twice for the reps' selling efforts. However, Proposition 4(a) and its proof show that the MD-C plan does not overpay reps for their selling efforts. Conversely, a possible concern arising from the activity metric is that sales reps may increase selling effort instead of thinking efforts to achieve activity commission $\left(w_{2}\right)$. If in fact the activity commission does not motivate reps to increase thinking efforts to improve selling effectiveness, then the MD-C plan will not serve its intended purpose. Yet, my Proposition 4(b) establishes that the activity commission motivates reps to choose more thinking efforts than under the standard compensation plan. Further, Proposition 4(c) shows that the multidimensional compensation plan achieves its goal to increase reps' thinking efforts more than the standard compensation plan does.

Proposition 4 contributes to sales management because it implies that the multidimensional compensation alters the firm's culture from one of only selling hard to one of enhancing selling effectiveness along with sales increases. In contrast, the standard compensation plan is not able to motivate reps to enhance their selling effectiveness because the incentive on sales does not influence sales reps' thinking effort choices. There is a reason why salespeople consider thinking more to maximize their utility under the MD-C plan. The incentive on activity that stimulates extra thinking effort $\left(e_{m c}>e_{s c}\right)$ directly overcomes the rep's disutility associated with thinking efforts. In the process, the activity incentive signals to the rep that the firm's goal is not just increasing sales but also improving the rep's selling effectiveness.

Next, Proposition 5 indicates that the outcomes realized under the optimal MD-C plan are superior to those obtained from the optimized standard compensation (SC) plan derived from the traditional PA model that assumes fixed selling effectiveness. Because of the complexity of the parameters, I provide support for proposition 5 via a numerical simulation of one scenario of plausible values of parameters involved. 
Proposition 5. Comparison of $S C$ and $M D-C$ compensation plans under proposed new model

All else equal,

(a) SC plan offers less incentive on sales than MD-C plan $\left(w_{s c, 1 j}^{*}>w_{m c, 1 j}^{*}\right)$ for $\forall \delta$.

(b) Sales rep $j$ will put more effort under MD-C plan than $S C$ plan $\left(x_{s c, 1 j}^{*}>x_{m c, 1 j}^{*}\right)$ for $\forall \delta$.

(c) The optimal thinking effort level under $M D$-C plan is higher than the optimal level under SC plan $\left(e_{s c, 1 j}^{*}<e_{m c, 1 j}^{*}\right)$, and so selling effectiveness will be higher under $M D-C$ plan than $S C$ plan $\left(\beta_{s c, 1 j}^{*}<\beta_{m c, 1 j}^{*}\right)$ for $\forall \delta$.

(d) The firm will achieve higher sales revenue under MD-C plan than SC plan $\left(s_{s c, 1 j}^{*}<\right.$ $\left.s_{m c, 1 j}^{*}\right)$ when $\delta_{j}<\bar{\delta}_{j}$ where $\bar{\delta}_{j}=\sqrt{\frac{c_{j}\left(\gamma_{1 j} \phi_{j}^{2}+\gamma_{2 j} u_{j}^{2}+\left(\gamma_{1 j} c_{2 j}+\gamma_{2 j} c_{1 j}\right) \mu_{j} \sigma_{s j}^{2}-u_{j} \phi_{j}\left(\gamma_{1 j}+\gamma_{2 j}\right)\right)}{\gamma_{1 j} c_{2 j} u_{j}+\gamma_{2 j} c_{1 j} \phi_{j}-c_{j}\left(\gamma_{1 j} c_{2 j}+\gamma_{2 j} c_{1 j}\right)}}$.

Proof: See appendix 6.1.6.

First, Proposition 5(a) focuses on a comparison of the firm's optimal incentive under the SC and MD-C plans. It notes that the SC plan's optimal sales incentive $\left(w_{s c, 1 j}^{*}\right)$ is higher than that of the MD-C plan $\left(w_{m c, 1 j}^{*}\right)$ regardless of the rep's level of base selling ability. This is attributable to the MD-C compensation plan incorporating two incentive parameters $\left(w_{m c, 1}, w_{m c, 2}\right)$ while the SC plan has only one incentive $\left(w_{s c, 1}\right)$. Thus, $w_{s c, 1}^{*}$ is larger than $w_{m c, 1 j}^{*}$ by as much as $\left(c_{1 j} \gamma_{2 j} \phi_{j}+c_{2 j} \gamma_{1 j} u_{j}\right)^{2}$. Consequently, the sales rep's optimal selling effort will be higher under the SC plan than the optimal level under the MD-C plan (proposition 5(b)). Notably, however, the selling effectiveness under the MD-C plan $\left(\beta_{m c, 1 j}^{*}\right)$ is higher than the selling effectiveness under the SC plan $\left(\beta_{s c, 1 j}^{*}\right)$ since the MD-C plan motivates reps to put in more thinking efforts $\left(e_{m c, 1}^{*}, e_{m c, 2}^{*}\right)$ by offering the activity incentive $\left(w_{m c, 2 j}\right)$ (proposition 5.(c)). Note that Table 3 shows the optimal thinking efforts under the SC plan are $e_{s c, 1 j}^{*}=\frac{u_{j}}{c_{j}} c_{1 j} \delta_{j}, e_{s c, 2 j}^{*}=\frac{\phi_{j} c_{j}}{c_{2 j} \delta_{j}}$ compared to the optimal levels under the MD-C plan are $e_{m c, 1 j}^{*}=\frac{u_{j} c_{j}}{c_{1 j} \delta_{j}}+\frac{c_{j} \gamma_{1 j}}{c_{1 j} \delta_{j}} \frac{w_{c c, 2 j}^{*}}{w_{m c, 1 j}^{*}}, e_{m c, 2 j}^{*}=\frac{\phi_{j} c_{j}}{c_{2 j} \delta_{j}}+\frac{c_{j} \gamma_{2 j}}{c_{2 j} \delta_{j}} \frac{w_{m c, 2 j}^{*}}{w_{m c, 1 j}^{*}}$.

Consequently, as $\left(s_{j}^{*}=x_{j}^{*} \beta_{j}^{*}\right)$ I obtain a condition for when the optimal sales rev- 
enue under the MD-C plan is greater than that under the SC plan (Proposition 5(d)). If a firm has more reps with $\delta_{j} \geq \bar{\delta}_{j}$, the SC plan will result in higher optimal sales revenue than the MD-C plan. If a firm has more reps with $\delta_{j}<\bar{\delta}_{j}$, the MD-C plan leads to higher optimal sales revenue than SC plan. However, which compensation plan will produce higher expected profits for the firm is uncertain. This is because a rep's expected wage under the MD-C plan may be lower or higher than that under the SC plan $\left(E\left[w_{s c, j}\right] \gtrless E\left[w_{m c, j}\right]\right)$. This inequality, unfortunately, is the 8th order function of $\delta$ and so it cannot be determined analytically considering the number of parameters involved. Therefore, the firm's profit which is a function of reps' wages, sales, and activity costs is also indeterminate and so the profits under MD-C plan may be smaller or larger than the one under SC plan $\left(\pi_{s c, j} \gtrless \pi_{m c, j}\right)$.

Nonetheless, the above findings are interesting because they are counterintuitive. The MD-C plan that has two incentive pay components intuitively seems like one that always offers more wages than the SC plan, Indeed, firms may judge that activity incentive is an "over-payment" when no additional sales or profit follows right away. Because of the over-payment concern, firms may not want to pay incentive on activity. However, when the activity incentive increases reps' selling effectiveness, it is no longer over-payment. Notably, the optimal MD-C plan leads to higher sales and profits than the SC plan and, therefore, there is no reason not to use MD-C plan.

\subsection{Standard-Uniform plan}

In practice, many firms choose to use a 'uniform' optimal incentive rate for all their salespeople even though they may be heterogeneous in various ways. Aside from it being simpler to set and administer a uniform plan, firms often do this due to asymmetric information problems that prevent customized plans or for the sake of appearing equitable. The uniform incentive plan's disadvantage, however, is that it may lead highly able reps to work less than under the standard customized plan. 
Below, I analyze performance in the standard-Uniform (SU) plan scenario when selling effectiveness is adaptable. More specifically, I assume the firm has two reps with different base abilities and compare their performances and the firm's outcomes under $\mathrm{SU}$ and SC plans. Let the uniform incentive plan for rep $\mathrm{j}$ be specified as:

$$
\bar{W}_{s u, j}=w_{s u, 0 j}+\bar{w}_{s u, 1} S_{j}
$$

where $w_{s u, 0 j}$ is the rep's basic salary, $\bar{w}_{s u, 1 j}$ is the uniform sales incentive under standard-uniform plan. Now suppose the two reps' base selling abilities are respectively $\delta_{1}>\delta_{2}$ where $\delta_{j} \in \mathbb{R}_{\geq 0}$. To simplify computation, let rep 1's ability level be twice rep 2's ability level $\left(\frac{1}{2} \delta_{1}=\delta_{2}=\delta\right)$. Suppose the corresponding values of all remaining parameters of the reps $\left(c, c_{1}, c_{2}, v, \mu, \sigma^{2}, u, \phi\right)$ are equal.

Proposition 6. Comparison of $S C$ and $S U$ plans

All else equal,

(a) Firm's total optimal thinking efforts from two reps under SU plan is equal to the corresponding total under SC plan $\left(\sum e_{s c}^{*}=\sum \bar{e}_{s u}^{*}\right)$ for $\forall \delta$.

(b) Firm's optimal commission rate on sales for high (low) base ability rep under SC plan is larger (less) than the common optimal sales incentive under SU plan $\left(w_{11, s c}^{*} \geq \bar{w}_{1, s u}^{*} \geq w_{21, s c}^{*}\right)$ if the low base ability reps' basic ability is large enough $(\delta \geq \hat{\delta})$

(c) Firm's total optimal selling efforts from two reps under SC plan is larger than the total optimal selling efforts under SU plan $\left(\sum x_{s c}^{*} \geq \sum \bar{x}_{s u}^{*}\right)$ if $\hat{\delta} \leq \delta \leq \sqrt{\frac{c b}{2 c_{1} c_{2}}}$. However, two reps' total optimal selling efforts under SC plan is less than the total optimal selling efforts under SU plan $\left(\sum x_{s c}^{*}<\sum \bar{x}_{s u}^{*}\right)$ if $\sqrt{\frac{c b}{2 c_{1} c_{2}}}<\delta$ or if $\delta<\hat{\delta}$.

(d) Firm's total optimal sales from two reps under SC plan is larger than the optimal sales under $S U$ plan $\left(\sum s_{s c}^{*} \geq \sum \bar{s}_{s u}^{*}\right)$ if the low ability rep's base ability is large enough $(\delta \geq \hat{\delta})$. Otherwise, $\sum s_{s c}^{*}<\sum \bar{s}_{s u}^{*}$. 
where $\hat{\delta}=\frac{-3 c \mu \sigma_{s}^{2}}{4 v}+\sqrt{\frac{c b}{2 c_{1} c_{2}}+\left(\frac{3 c \mu \sigma_{s}^{2}}{4 v}\right)^{2}}, b=c_{1} \phi^{2}+c_{2} u^{2}+c_{1} c_{2} \mu \sigma_{s}^{2}$

Proof: See appendix 6.1.7.

Under SC and SU plans, the sales reps' selling effectiveness does not change because the sales incentive does not influence any thinking efforts. In other words, the totals of optimal thinking efforts from two reps under SC plan and SU plan are equal (proposition 6.(a)). This implies the firm can only impact the reps' optimal selling efforts via the sales commission rate (Proposition 6(b)). Then, if the two reps' base abilities are high enough $(\delta \geq \hat{\delta})$, the firm's SC plan should follow the traditional logic, i.e., offer a higher customized sales incentive to the rep with higher base ability (Proposition 6(c)). It follows that the more (less) able sales rep 1 (2), will put in more (less) selling effort under the SC plan than under the SU plan $\left(x_{1}^{*}>\bar{x}_{1}^{*}\right)($ $\left.\left(x_{2}^{*}>\bar{x}_{2}^{*}\right)\right)$. Hence, the firm's total selling effort from the two reps depends on the difference between their optimal selling efforts $\left(\left|x_{1}^{*}-\bar{x}_{1}^{*}\right| \lessgtr\left|x_{2}^{*}-\bar{x}_{2}^{*}\right|\right)$. I find that when the low ability rep 2's base selling ability level is moderate $\left(\hat{\delta} \leq \delta \leq \sqrt{\frac{c b}{2 c_{1} c_{2}}}\right)$, the total selling effort from two reps obtained by the firm under SC plan is larger than under SU plan.

However, when the low ability rep 2's base ability is fairly high $\left(\sqrt{\frac{c b}{2 c_{1} c_{2}}}<\delta\right)$, rep 1's increased amount of selling effort under SC plan is smaller than rep 2's increased amount of selling effort under SU plan $\left(\left|x_{1}^{*}-\bar{x}_{1}^{*}\right|<\left|x_{2}^{*}-\bar{x}_{2}^{*}\right|\right)$. In this scenario, the total selling effort from the two reps under the SC plan can be smaller than under the SU plan.

Interestingly, however, when $\delta \geq \hat{\delta}$, the total sales revenue under SC plan will always be larger than the production under the SU plan (Proposition 6(d)) even when firm's total optimal selling efforts are smaller under SC plan than under SU plan $\left(\delta>\sqrt{\frac{c b}{2 c_{1} c_{2}}}\right)$. This is because when both reps' base abilities are very high, i.e., when both have sufficiently high selling effectiveness, the firm will always achieve higher sales under SC plan than under SU plan. This analysis is consistent with 
Daljord et al. (2016)'s finding that customized compensation plan results in the firm having better outcomes than those under a uniform compensation plan.

Now, let us consider the case when neither rep's base selling abilities are very high

$(\delta<\hat{\delta})$. In this case, the customized optimal commission rate for the more able rep 1 is smaller than the optimal uniform commission rate as well as the customized rate for the less able rep $\left(w_{11, s c}^{*}<\bar{w}_{1, s u}^{*}<w_{21, s c}^{*}\right)$. Also, the total selling efforts from the two reps under SU plan is larger than the level under the SC plan. Intuitively, the more able rep 1 is not motivated as much by his $\mathrm{SC}$ commission rate as he is by the SU commission rate because $\left(w_{11, s c}^{*}<w_{21, s c}^{*}\right)$. As a result, the firm's total optimal sales from the two reps under the SC plan becomes smaller than the level under SU plan. This case implies that there can be an adverse effect of SC compensation plan if the salespeople's base abilities are too low.

Regardless of whether SU or SC plan is superior, neither can enhance selling effectiveness (Proposition 6(a)). As long as the compensation planners believe that reps' selling effectiveness $(\beta)$ is fixed they may stick to a standard only sales-based plan. However, considering that firms invest in sales training programs implies that practitioners do believe that reps' selling effectiveness is adaptable. Then, as I have shown in Proposition 6, under any form of standard plan, the reps' optimal choices of thinking efforts during the selling process will not be altered and selling effectiveness is not impacted. Therefore, if firms believe that reps' selling effectiveness is adaptable and wish to influence it, the multidimensional compensation plan including an activity incentive which can affect choice of thinking efforts becomes necessary.

\subsection{Multidimensional-Uniform Plan}

Lastly, I investigate the Multidimensional-Uniform (MD-U) compensation plan which is a more easily applicable plan than the MD-C compensation plan. The MD-U plan 
would be represented as:

$$
\bar{W}_{m u, j}=w_{m u, 0 j}+\bar{w}_{m u, 1} S_{j}+\bar{w}_{m u, 2} K_{j}
$$

where $w_{m u, 0 j}$ is rep j's basic salary, $\bar{w}_{m u, 1}$ is the uniform sales incentive, and $\bar{w}_{m u, 2}$ is the uniform activity incentive under the MD-U plan. In Proposition 7, I summarize the key differences between results under the MD-C versus MD-U models. Similar to what I assumed in developing Proposition 6, suppose that rep 1's ability is twice that of rep 2's ability $\left(\frac{1}{2} \delta_{1}=\delta_{2}=\delta\right)$, and all the other parameter values $\left(c, c_{1}, c_{2}, v, \mu, \sigma^{2}, u, \phi\right)$ are the same across the two reps.

Proposition 7. Comparison of $M D-C$ and $M D-U$ Plans

All else equal,

(a) Firm's optimal commission rate on sales for high (low) base ability rep under $M D-C$ plan is larger (less) than the common optimal sales incentive under $M D-U$ plan $\left(w_{11}^{*} \geq \bar{w}_{1}^{*} \geq w_{21}^{*}\right)$ if the low ability rep's basic ability is large enough $(\delta \geq \check{\delta})$.

(b) Firm's optimal commission rate on activity for high (low) base ability rep under $M D-C$ plan is lower (larger) than the common optimal activity incentive under $M D-U$ plan $\left(w_{22}^{*} \geq \bar{w}_{2}^{*} \geq w_{12}^{*}\right)$ if the low ability rep's basic ability is large enough $(\delta \geq \check{\delta})$.

(c) Firm's total optimal selling efforts from the two reps under $M D$-C plan is larger than the one under $M D-U$ plan $\left(\sum x_{m c}^{*}>\sum \bar{x}_{m u}^{*}\right)$ if $\check{\delta}<\delta<\sqrt{\frac{c\left(\hat{a}+\hat{b} \mu \sigma_{s}^{2}\right)}{2 \hat{b}}}$. Otherwise, firm's total optimal selling efforts from the two reps under $M D$-U plan is larger than that under $M D-C$ plan $\left(\sum x_{m c}^{*}<\sum \bar{x}_{m u}^{*}\right)$.

(d) Firm's total optimal thinking efforts from the two reps under MD-C plan is larger than that under $M D-U$ plan $\left(\sum e_{m c}^{*}>\sum \bar{e}_{m u}^{*}\right)$ if $\delta>\check{\delta}$. Otherwise, firm's total optimal thinking efforts from the two reps under $M D$ - $U$ plan is larger than the corresponding total under $M D-C$ plan $\left(\sum e_{m c}^{*}<\sum \bar{e}_{m u}^{*}\right)$.

(e) Firm's total optimal total sales from the two reps under MD-C plan is larger than 
the optimal total sales under $M D-U$ plan $\left(\sum s_{m c}^{*} \geq \sum \bar{s}_{m u}^{*}\right)$ if the less able rep's basic ability is large enough $(\delta \geq \check{\delta})$ and thinking effectiveness for enhancing selling effectiveness is larger than for improving activity $\left(1<\gamma_{1}^{2} c_{2}\right.$ and $\left.1<\gamma_{2}^{2} c_{1}\right)$. Otherwise, $\sum s_{m c}^{*}<\sum \bar{s}_{m u}^{*}$

where $\check{\delta}=\frac{-3 c \mu \sigma_{s}^{2}}{4 v}+\sqrt{\frac{c\left(\hat{a}+\hat{b} \mu \sigma_{s}^{2}\right)}{2 \hat{b}}+\left(\frac{3 c \mu \sigma_{s}^{2}}{4 v}\right)^{2}}, \hat{a}=\left(\phi \gamma_{1}-u \gamma_{2}\right)^{2}+\left(c_{1} \phi^{2}+c_{2} u^{2}\right) \mu \sigma_{k}^{2}$, $\hat{b}=c_{2} \gamma_{1}^{2}+c_{1} \gamma_{2}^{2}+c_{1} c_{2} \mu \sigma_{k}^{2}$.

Proof: See appendix 6.1.8.

Proposition 7(a) is consistent with the result in Proposition 6(a). When the low ability rep 2's base ability is large enough $(\delta \geq \check{\delta})$, the firm should give more sales incentive to the high ability rep 1 than to low ability rep 2 under MD-C plan. Next, Proposition $7(\mathrm{~b})$ says the firm should give higher activity incentive to the low ability rep 2 than to rep 1 under MD-C plan in contrast to the common activity incentive under MD-U plan. Further, the firm's optimal incentive strategy using the MD-C plan is to provide higher sales commission rate to the more able rep and a higher activity incentive rate to the less able rep.

Further, if the less able rep's base ability is moderately high $\left(\check{\delta}<\delta<\sqrt{\frac{c\left(\hat{a}+\hat{b} \mu \sigma_{s}^{2}\right)}{2 \hat{b}}}\right)$, the total optimal selling effort from the two reps under MD-C plan is larger than under MD-U plan (proposition 7.(c)). Under MD-C plan, the more able rep will put in higher selling effort than under MD-U plan while the less able rep puts in less selling effort than under MD-U plan. Overall, it can be seen that the total selling effort from the two reps obtained by the firm is higher under the MD-C plan than under MD-U plan.

On the other hand, if the lower base ability of rep 2 is sufficiently high $\left(\sqrt{\frac{c\left(\hat{a}+\hat{b} \mu \sigma_{s}^{2}\right)}{2 \hat{b}}}<\right.$ $\delta)$ and $(\delta<\check{\delta})$, the firm will obtain lower total optimal selling effort amount from the two reps under MD-C plan than under the MD-U plan. Basically, the more able rep does not increase her/his selling effort much due to diminishing returns while the less able rep will reduce selling effort because of the lower sales incentive (compared to 
what the rep would receive under the MD-U plan). Hence, this is a condition when the total selling effort under MD-C plan is lower than that under the MD-U plan.

Moreover, the total optimal thinking effort amount under MD-C plan is larger than MD-U plan when the low ability rep 2's base ability is sufficiently high (Pproposition $7(\mathrm{~d})$ ). Specifically, the lower (higher) ability rep's thinking effort under MD-C plan will be larger (smaller) than under MD-U plan. Interestingly, the lower base ability rep 2's optimal thinking effort amount is always larger than that of the high base ability rep 1 (see the appendix 6.1.8.d-3). This implies that the low base ability reps in the firm will show a bigger improvement in selling effectiveness when the firm adopts the optimal MD-C scheme.

Accordingly, the firm's total optimal sales from the two reps under MD-C plan will be higher than under MD-U plan when the low ability rep 2's base ability is large enough $(\delta>\check{\delta})$ and thinking effectiveness on activity and costs are large enough $\left(1<\gamma_{1}^{2} c_{2}\right.$ and $1<\gamma_{2}^{2} c_{1}^{2}$ ) (proposition 7.(e)). On the other hand, if the reps' thinking effectiveness in target activity achievement and associated costs are small $\left(1>\gamma_{1}^{2} c_{2}\right.$ and $1>\gamma_{2}^{2} c_{1}$ ), the firm's total optimal sales under MD-U plan can be higher than under the MD-C plan.

Lastly, if the low ability rep 2's base ability is not large enough $(\delta<\check{\delta})$, then the story reverses. Under MD-C plan, the high ability rep 1 should receive higher activity incentive to increase selling effectiveness and the low ability rep 2 should receive higher sales incentive to enhance selling effort (Proposition 7(a), (b)). Then, the firm's total optimal thinking efforts from the two reps under MD-U plan will be higher than under MD-C plan (Proposition 7(e)) as are the total optimal sales (Proposition 7(e)). These insights differ from the traditional view that outcomes under optimal customized plans are always superior to those under the uniform plan. However, due to reps' thinking effort as well as selling effort decisions in my new

\footnotetext{
${ }^{2} \gamma_{1,2}^{2}$ represent the convex thinking effectiveness on activity, $c_{1,2}$ represent the thinking cost, and all parameters are less than 1 . With multiplying $c_{1,2}$, the slope of $\gamma_{1,2}^{2}$ gets lower.
} 
model, it is possible for the firm to achieve better outcomes with a MD-U than with a MD-C incentive plan.

In conclusion, the above analyses shows how a firm should motivate reps who are heterogeneous in their selling ability to improve sales as well as selling effectiveness. However, in my comparative analyses of the four compensation scenarios I are unable to analytically show which compensation plan produces the highest expected profits for the firm. Therefore, I now turn to numerical simulations to shed more light on this issue.

\section{Numerical Simulation Analyses}

In the following numerical simulation analyses, I consider a firm with J number of heterogeneous reps with the basic selling ability of salesperson $\mathrm{j}, \mathrm{j}=1, \ldots, \mathrm{J}$ denoted by $\delta_{j} \in \mathbb{R}_{\geq 0}$. In these analyses I utilize realistic values of key parameters of the compensation design problem drawn from Daljord et al. (2016) to obtain more insights into the following questions : When reps' choices with respect to thinking efforts alter selling effectiveness, what are the outcomes for reps and the firm under the four compensation plan scenarios considered in the last section? Which plan option results in the best outcomes for the firm and the reps and under what conditions?

Table 6: Empirical Mean of Parameters from Daljord et al. (2016)

\begin{tabular}{|c|c|c|c|c|c|c|c|c|c|c|c|c|c|c|}
\hline Daljord et al. & $\begin{array}{l}\alpha \\
.9962\end{array}$ & $\begin{array}{l}\mu \\
.0314\end{array}$ & $\begin{array}{l}\bar{F} \\
.0864 \\
\end{array}$ & $1.0802^{\beta}$ & & & .0489 & $c$ & & .3114 & & & & \\
\hline This paper & .9962 & .0314 & .0864 & $\begin{array}{l}\delta \\
0.2-1.2\end{array}$ & $\begin{array}{l}u \\
.6\end{array}$ & $\begin{array}{l}\phi \\
.4\end{array}$ & $\begin{array}{l}l c \\
.0489\end{array}$ & $\begin{array}{l}l_{1} \\
.0636\end{array}$ & $\begin{array}{l}c_{2} \\
.0736\end{array}$ & $\begin{array}{l}\sigma_{S} \\
.3114\end{array}$ & $\begin{array}{l}\sigma_{K} \\
.0811\end{array}$ & $\begin{array}{l}\mathrm{v} \\
.21\end{array}$ & $\begin{array}{l}\gamma_{1} \\
1.1591\end{array}$ & $\begin{array}{l}\gamma_{2} \\
1.0811\end{array}$ \\
\hline
\end{tabular}

As I mentioned above, I adapted empirical estimates of key parameters of the problem from a field study of a sales force of 58 sales reps.provided by Daljord et al. (2016, Table 3, P. 171), namely base level of sales $(\mathrm{h}=\alpha)$, risk aversion $(\mathrm{r}=\mu)$, base level of selling effectiveness $(\mathrm{k}=\beta)$, disutility cost of selling effort $(\mathrm{d}=c)$, and reservation utility $\left(U_{0}=\bar{F}\right)$. Using Daljord et al. (2016)'s estimates of the ranges for 
each of these estimates, I simulated the parameter values for an arbitrarily sized set of 100 sales reps. For those parameters introduced for the first time in my model, e.g., thinking effort effectiveness parameters $(u, \phi)$, I use some plausible numbers. Table 6 shows the mean parameter values derived from Daljord et al. (2016) and those assumed for other parameters here for performing these simulation analyses.

In the following analyses, to keep the focus on the broad issues of interest noted above, I only vary the base level of selling ability $(\delta)$, holding all other parameter values fixed. I begin with the benchmark comparison of the results with a standardcustomized incentive plan under the traditional agency-theoretic model (that assumes selling effectiveness is fixed and equal to the base level of selling ability) and my new behavioral agency-theoretic model with adaptable selling effectiveness.

\subsection{Traditional vs New Model}
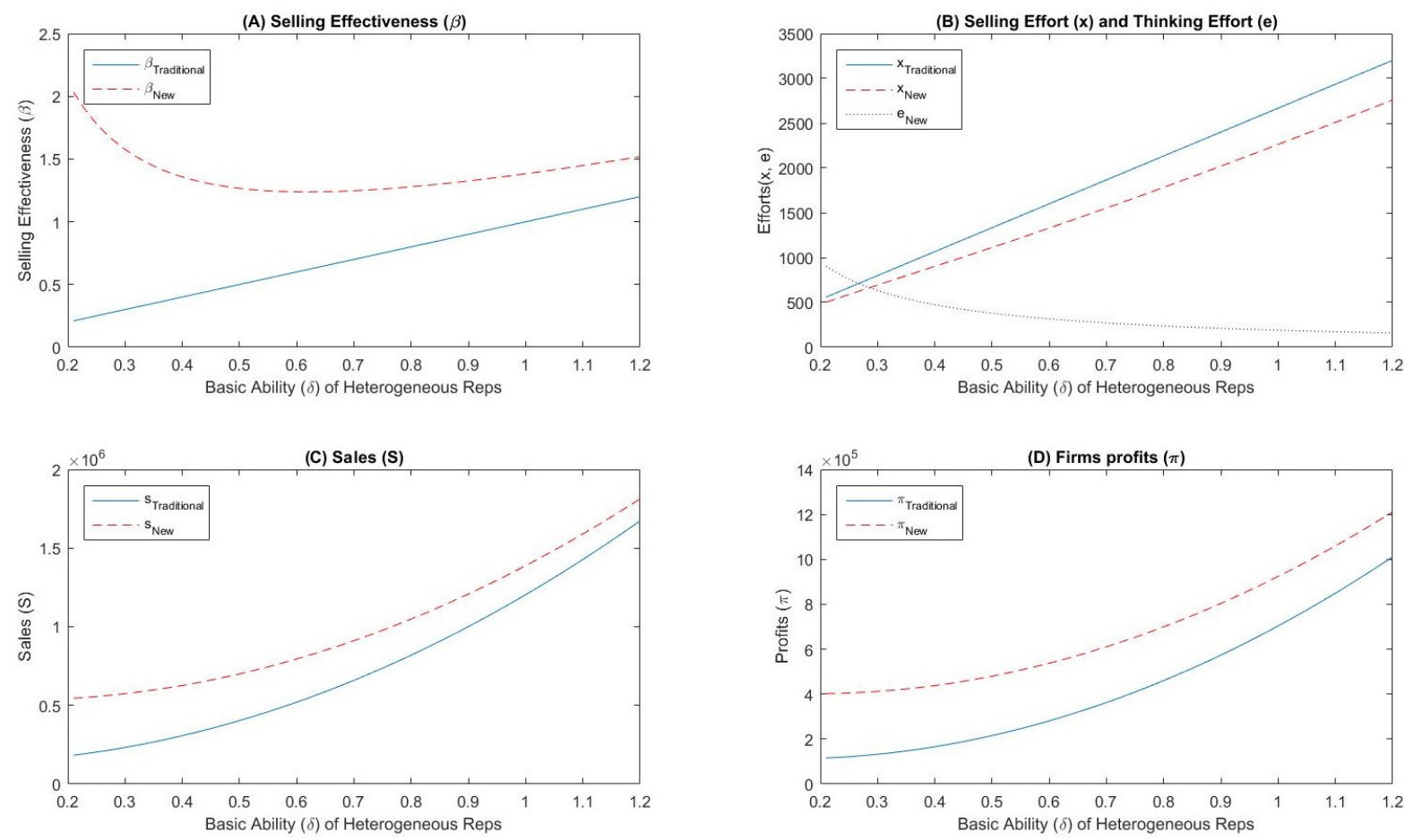

Figure 4: Comparison based on Heterogeneous Basic Ability between Traditional and New Model 

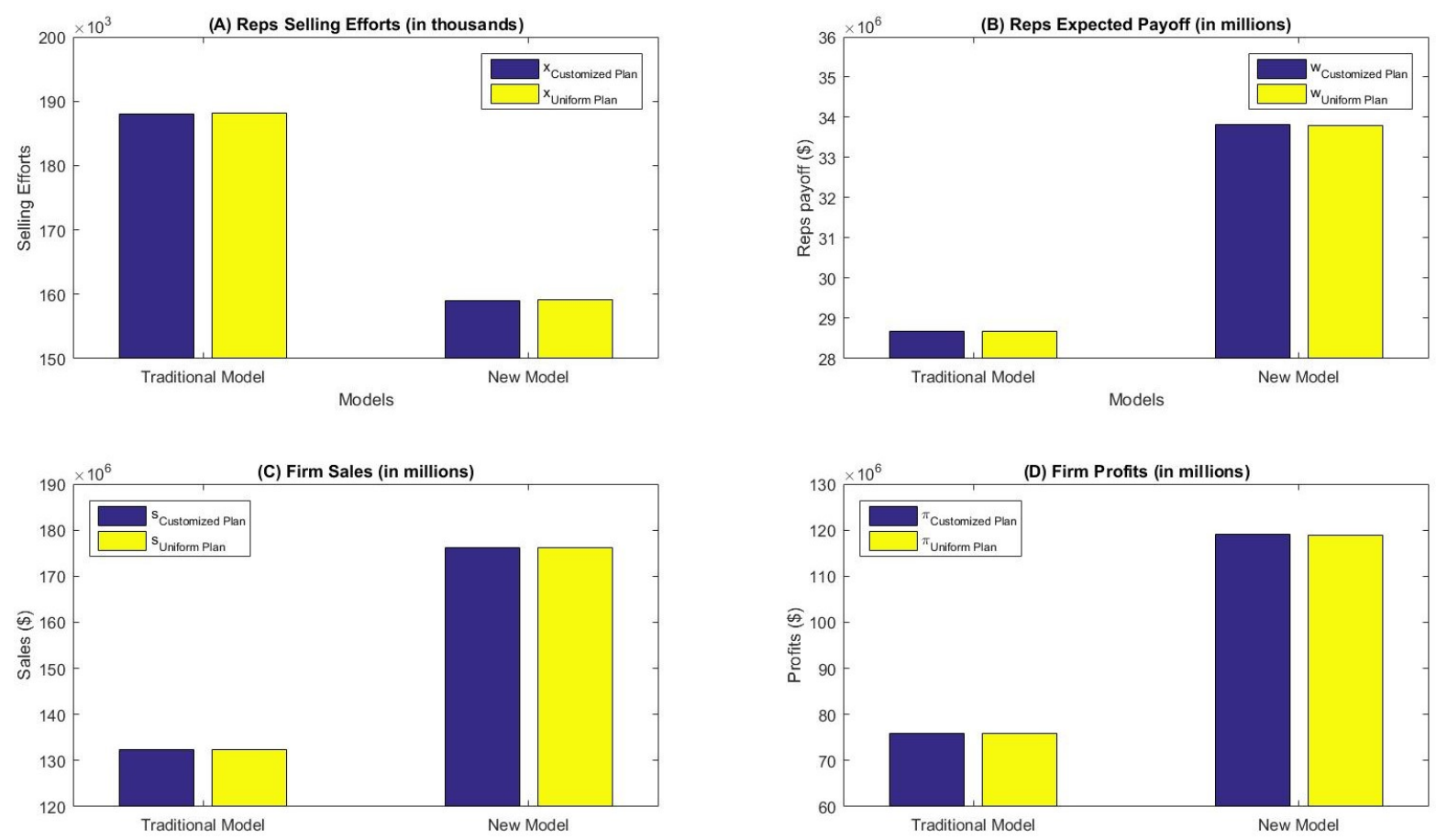

Figure 5: Cumulative Comparison between Traditional and New Model

Figure 4(A) shows that under the new model, selling effectiveness $\beta$ is not always proportionate to the base ability $\delta$. More specifically, when the value of $\delta$ is below 0.5 , overall selling effectiveness can be as high as 2.1. This occurs because sales reps with lower base ability put in more thinking efforts to maximize their expected utility (Figure 4(B)). Traditional models of course do not allow for this choice on improving selling effectiveness. This implies, as shown in the following Figures (5(A) and 5(B)), that outcomes can improve once you allow for adaptable selling effectiveness. Moreover, firms' outcomes can be superior even if salespeople choose to increase their thinking efforts rather than selling effort $(5(\mathrm{C})$ and $5(\mathrm{D}))$. This numeric simulation also supports the corresponding Proposition 2.

\subsection{Standard v Multidimensional Plan Results in New Model}

Figure 6(A) and (B) show that reps' total optimal thinking efforts for SC and SU plans are equal and it is consistent with the Proposition 6a. Reps' total optimal selling 
effort under SU (159,009 hrs) is slightly lower than under SC (159,208 hrs) (figure $6(\mathrm{C})$ ). Yet, the reps' total optimal sales (\$176.19 mil) under SU is about the same as that under SC ( $\$ 176.19$ mil) (figure $6(\mathrm{G})$ ) because low base ability reps put in more selling efforts than high base ability reps do under SU plan. Hence, reps' expected payoff under SC (\$33.81 mil) is somewhat larger than under SU plan (\$33.80 mil) and the total optimal profits under SC (\$119.01 mil) is also slightly larger than under SU (\$118.98 mil) (figure 6(E),(H)). Overall, it reveals that the firm’s performance under $\mathrm{SC}$ plan is superior to the results under SU plan (RQ2) although the difference is not much. Consequently, I confirm that the firm's outcomes in this numerical simulation are consistent with Proposition 6.c,d when $\sqrt{\frac{c b}{2 c_{1} c_{2}}}<\delta$.

The additional new insights are as follows. First, a multidimensional incentive plan (MD-C) yields superior results to standard incentive plan (SC) when reps' base ability vary at the firm. Specifically, sales reps choose higher thinking efforts under multidimensional plans than under standard plans (figure 6(A),(B)), but reps under MD-C plan produce less optimal selling efforts than under SC plan (figure 6(C), proposition 5.b). Due to high selling effectiveness by high thinking efforts under MDC plan, total optimal sales under MD-C plan (\$176.20 mil) are slightly higher than under SC plan (\$176.19 mil) (figure $6(\mathrm{G})$ ). However, the total optimal profit under MD-C plan ( $\$ 121.76$ mil) is higher than that under SC plan $(\$ 119.01$ mil) because of the total cost reduction (figure 6(F). Firm's total cost (figure 6(F)) includes selling activity costs (figure 6(D)) and reps' expected payoff (figure 6(E)). Since the MD-C plan minimizes the overall selling activity cost enough to offset the increase of reps' expected payoff, firm's profit under MD-C plan is higher than that under SC or SU plans.

Second, the empirical simulation demonstrates that the MD-U plan can produce results superior to MD-C plan. Since the MD-C plan induces reps to produce too much thinking efforts (figure 6(A),(B), Proposition $7(\mathrm{~d})$ ), reps under MD-C plan, 

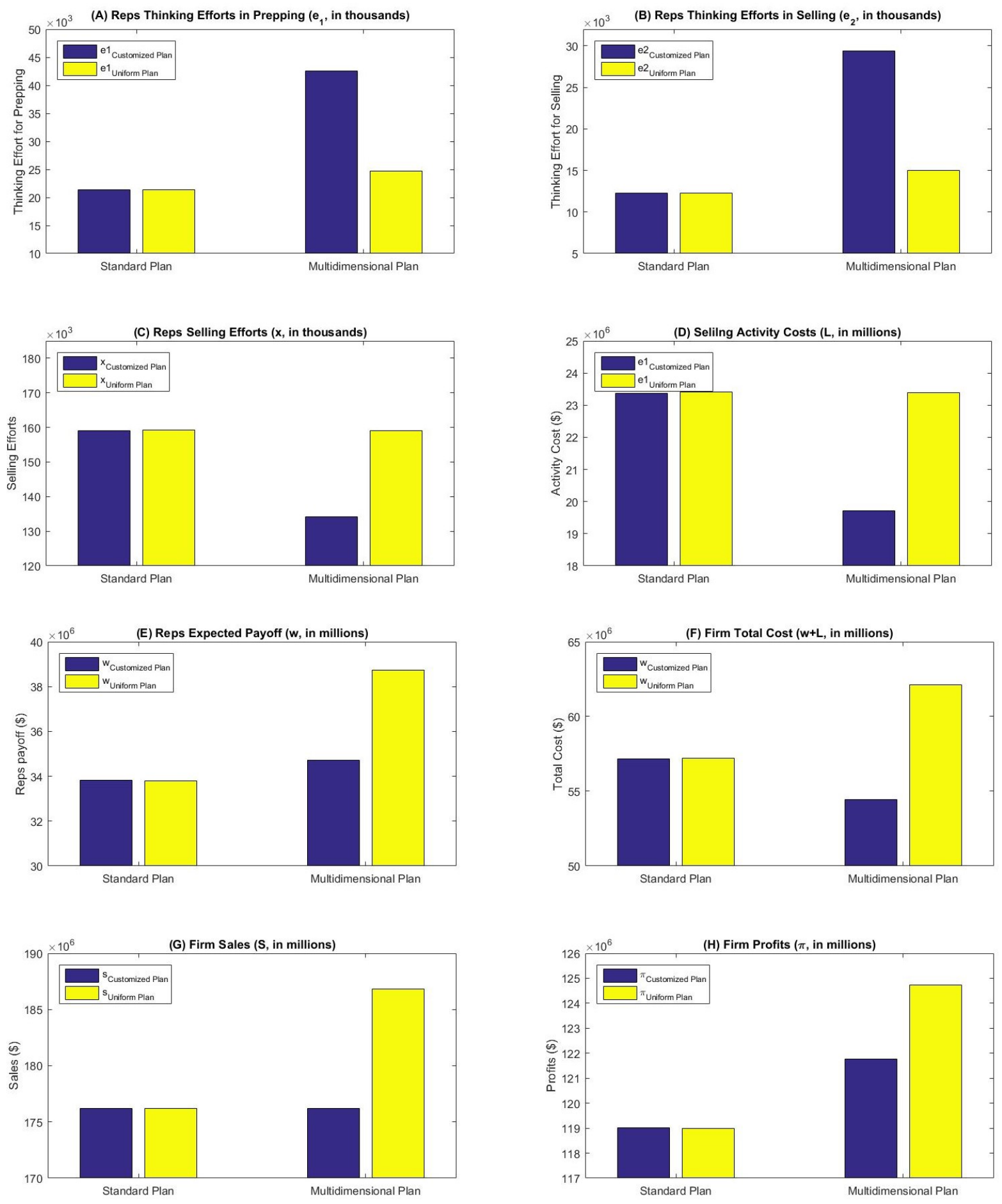

Figure 6: Comparison between Standard and Multidimensional Compensation 
consequently, put the least amount of selling effort (figure 6(C), proposition 7.(c)). However, MD-U plan alleviates the extreme increase in thinking efforts and decrease in selling effort. Consequently, total cost under MD-U plan is the highest (figure 6(G)) because reps' expected payoff is the highest (figure 6(E)). However, managers should be aware that the increase of payoff under the MD-U plan is because of enhanced effectiveness without reduction of selling effort and, as a result, it stimulates the highest sales. The total optimal sales under MD-U plan (\$186.85 mil) is higher than under MD-C plan (\$176.20 mil) (figure 6(G), Proposition 7(e)) and the other standard plans. Further, total optimal profit under MD-U plan (\$124.74 mil) is also higher than under MD-C plan (\$121.76 mil) (figure 6(H)).

Therefore, I conclude that MD-U plan which produces from highest sales outcome and profits than other compensation plans by motivating reps to increase both selling effort and selling effectiveness. This is a surprising new insight that emerges from my analyses.

\section{Conclusion}

Under any compensation plan, sales reps adjust their effort strategy to achieve their maximum utility. Hence, the design of compensation plans that induce the right combination of efforts is a critical issue for sales management. In this paper, I introduce the key notion of adaptable selling effectiveness, via variable thinking efforts, into model-based sales force compensation design. Using my "New Model" I investigate how sales reps will respond to four different types of compensation plans: standardcustomized, multidimensional-customized, standard-uniform, and multidimensionaluniform.

In contrast to the fixed selling effectiveness model, the new model proves that high base ability reps under standard compensation plan may produce lower profits than 
low base ability reps because the reps with low base ability may improve selling effectiveness by putting in more thinking efforts. Also, under the standard compensation plan, optimal thinking effort and selling effectiveness do not depend on uncertainty and reps' risk aversion. Instead, high selling effort cost, low thinking cost, and high thinking effectiveness increase reps' selling effectiveness. Hence, when a firm uses a standard compensation plan, hiring reps with high thinking effectiveness or low cost of thinking is recommended rather than reps with only high base ability. On the other hand, under the multidimensional compensation plan, uncertainty and selling activity cost increase reps' selling effectiveness, because of the activity incentive. Moreover, the multidimensional compensation plan can produce results superior to the standard sales-based plan because salespeople choose to increase selling effectiveness only under multidimensional plan. More importantly, I emphasize that selected activity metrics in such plans must stimulate the kind of thinking efforts involved in 'prepping' and 'selling' stages of the selling process that enhance selling effectiveness. Counter to conventional wisdom, a uniform plan across the sales force can yield outcomes superior to those under customized compensation plans when salespeople's base ability is moderate.

From a managerial viewpoint, my proposed model framework can be used to optimally structure sales plus activity-based sales force incentive plans that can be uniform or customized. Until now, a formal framework like ours for designing such multidimensional sales force incentive plans has been missing in the sales management research literature. In practice, typically, sales-based incentives and activity-based bonuses are set in piecemeal fashion leading to suboptimal results. My proposed model in this paper can assist managers to set such sales force incentives in a more systematic and integrated fashion. Using this framework, I attain intriguing managerial insights on salespeople's behavior and firm strategy.

Future research can extend this paper in many directions. For example, it would 
be valuable to investigate and compare results under alternative forms of multidimensional compensation plans as well as derive the optimal form under general conditions. The first order of business on my own research agenda, however, is to perform more empirical studies of assumptions and tests of propositions emerging from my proposed New Model. Second, sales managers in practice use many assorted activity metrics and there is a need to assess and establish the most suitable activity metrics when the firm's objective is to enhance selling effectiveness as well as increase sales. Last but not least is the goal of designing and validating an implementable decision support system for setting optimal multidimensional compensation plans in practice. 


\section{CHAPTER III: Essay 2}

\section{Compensation Mechanism for Coordinating Inside and Outside Sales Forces}

\section{Introduction}

In 2017, InsideSales.com reported that $47.2 \%$ (2.7 million) of 5.7 million is Inside Sales Rep in the U.S. Business-to-Business (B2B) firms and they expect that the number of the Inside Sales Rep would increase up to 50\%. Moreover, Inside Sales Rep (ISR) became an independent sales team such as Outside Sales Rep (OSR) who sells products and services to buyers (Arli et al. 2018). Consequently, the increasing ISR's role challenges B2B firms' structure which traditionally depends on OSR's sales outcome. One of the biggest challenges for many B2B selling firms is to find a way to coordinate and ensure that ISR and OSR work cooperatively (Arli et al. 2018). Hence, this paper will investigate the ISR and OSR's optimal cooperative effort strategy and the firm's outcomes under the suggested Target-Value wage system.

The reason ISR became an independent sales team results from B2B buyers' purchasing behavior change. As technology has developed, B2B buyers started to search for information to purchase products proactively (Steward et al. 2019, Toman et al. 2017). For example, B2B buyers exploit all necessary information to purchase complex products by searching the internet, reading white papers/E-books, visiting Vendor Web sites, participating in Webinars, or asking questions through social media (Toman et al. 2017, Mantrala and Albers 2012). The B2B buyers, then, approach the selling firm's ISR to purchase. Consequently, ISRs became interacting with the prospective B2B buyers using online at a higher rate (Grewal et al. 2015, Mantrala 
2014). According to the report of Salesforce.com, new ISR's role has increased up to $7 \%$ since 2015 (Salesforce.com). In contrast, OSR's sales has diminished (Steenburgh et al. 2009) because the traditional selling approach, such as cold calling, does not attract B2B buyers who behave proactively.

As ISR's position becomes essential as much as OSR's position, consequently, two serious problems would arise. First, ISRs and OSRs would not cooperate actively. Traditionally, ISRs worked for OSRs as administrative staffs (e.g., call centers, telemarketers) (Bendapudi and Leone 2002) and ISRs were not primarily involved in prospecting or closing sales (Shi et al. 2017). Hence, OSRs who are familiar with the traditional sales team structure would not consider ISRs as professional salespeople or have the willingness to cooperate with the independent ISR. In a case that a hybrid team structure is appropriate and well-organized, ISRs and OSRs would share their team-incentives and work cooperatively (Arli et al. 2018). In many cases, however, ISRs take a more independent sales role with remote selling based on inbound buyers' contacts and information (Salesforce.com), and expect to receive their sales-based incentives (Kundu 2019). Due to the difference in their positions, therefore, ISRs and OSRs would pursue their sales commissions and benefits rather than the firm's profits.

Sequentially, it leads to the second issue, inappropriate service and poor B2B customer's experience. For example, lets' assume that a potential consumer who prefers remote communication in order to ask about services and products calls a B2B firm's call center. Also, lets' presume that the call center connected this potential consumer to OSR as usual. However, OSR would not be the appropriate channel to determine and sell the proper product to this consumer. It is because OSR, who is the expert of face-to-face interaction, is likely to misinterpret the consumers' preferences or interests when they communicate online. If the OSR misjudges the potential consumer as who is not interested in their products, OSR would neglect the potential consumer and focus on another potential consumer to increase his/her own sales 
earnings. Then, the neglected potential consumer would be disappointed and leave to search for another firm (Strategies 2019). If the OSR pushes to sell products remotely by himself/herself to the prospective consumer, OSR is likely not to be successful because he/she is not good at remote communication as much as the ISR is (Sincavage 2019). However, there is one way not to lose this potential customer. The OSR can delicately pass on the potential customer to an appropriate ISR, which is the preferred path for this potential consumer and would successfully result in a sale.

Nonetheless, the OSR is highly likely to leave the potential customer or to push the deal directly if there is no incentive for the effort to delicately handing over the customer to ISR. More specifically, a common standard compensation plan (e.g., salary plus incentive on sales) does not reward OSR from handing over the potential customer to the ISR. If the OSR spends time to hand over the customer to the ISR with no incentive, OSR would think that it is wasting time instead of selling products to his/her own higher potential consumers. Hence, under standard compensation, the OSR's choice is very likely to leave the potential customer or push the deal. For ISR's case, vice versa.

Then, the question is how the firm motivates ISR and OSR to choose to hand over potential customers when necessary. There are various ways to motivate multifunctional people to cooperate for consumers' better experience; monitoring and encouraging cooperation (Dixon et al. 2002), organizing hybrid sales team (Arli et al. 2018), developing intraorganizational relationships to increase collaboration (Arli et al. 2018), and compensating (Hauser et al. 1996). However, managerially monitoring ISRs and OSRs requires high costs. Also, organizing of hybrid sales team with a team-selling incentive is not a new idea. Several studies have shown that team-based selling leads to positive team outcomes, team performance, firms' coordinated strategy, cross-selling goal, and customer relationship improvement (Moon 
and Armstrong 1994, Mohammed and Dumville 2001, Moorman and Albrecht 2008, Ahearne et al. 2010a, Schmitz 2013, Rapp et al. 2014). Moreover, ISR and OSR mostly produce independent sales outcomes because of different consumers' needs. Hence, developing intraorganizational relationships would not be the answer. Overall, therefore, one of the proper approaches is offering a new form of joint incentive mechanism that motivates the qualification and exchange of consumer leads between ISR and OSR.

Extant principal-agency theoretical model papers have investigated the optimal structuring under various conditions such as heterogenous salespeople, multi-products, multi-territories (Basu et al. 1985, Lal and Srinivasan 1993, Raju and Srinivasan 1996, Mantrala et al. 1997, Joseph and Thevaranjan 1998, Kalra et al. 2003, Murthy and Mantrala 2005, Rubel and Prasad 2015, Schöttner 2016). However, there is no sales compensation research considering cooperation among independent multiple sales teams. Uniquely, Hauser et al. (1996) consider compensation using peer evaluation in $R \& D$ and marketing team context, and they show that a target-value compensation system using peer evaluation can induce optimal agents' efforts. Hauser et al. (1996) also prove that only a profit outcome-based compensation system is hard to satisfy them fully. Although their goal is not purely independent sales teams' cooperating, their paper shows the possibility of using behavior-based compensation for enduing cooperating behavior. Hence, the target-value compensation system would be more feasible to enforce ISR and OSR collaborating than monitoring salespeople or hybrid structuring.

To summarize, there is no existing sales force compensation literature to induce cooperation among independent multiple sales groups (e.g., ISR and OSR) and pursue a firm's profit. To address this gap in the sales compensation literature and provide managerial implication with regard to adapting the Target-Value wage system, we will show and discuss the two different sales groups' optimal effort and firm outcomes 
under the Target-Value wage. There are two objectives of this paper analysis to answer the following questions: (1) Can the proposed Target-Value wage, rewarding reps for own sales, cooperative sales, cooperating activity and penalizing deceitful activity, increase both reps' and the firm's payoffs? (2) How do ISR and OSR performance respond differently to Target-Value wage, outcome-based standard wage, and multidimensional activity-based incentive wage? Subsequently, this paper will show that ISR and OSR will increase cooperative working effort under both Target-Value wage and multidimensional activity-based incentive wages. Moreover, Target-Value wage prohibits the salespeople's deceitful behavior. Eventually, both salespeople and firms will achieve better outcomes under Target-Value and multidimensional wages than outcome-based standard wage.

The rest of the second essay is organized as follows. In the next section 2, I review the literature in ISR sales papers, team compensation, and behavior-based and outcome-based control systems. In section 3, I show my modified Target-Value wage system and solve for an optimal firm and reps' actions and payoffs. In section 4, I analyze the optimal solutions and discuss new propositions and insights using comparative statics, and in section 5, I extend the analysis of two other alternative compensation mechanisms with numerical simulation analyses. Finally, I will summarize my research contributions, managerial implications, and suggested directions for future research in the last section.

\section{Literature Review}

Research on the new role of ISR (e.g., from qualifying leads to remote selling) is scarce and there is none of ISR's compensation research. Thaichon et al. (2018) argue that the ISR's role became significant due to the e-commerce and online information development in 2000s. Specifically, they argue that customers actively contact ISR to 
clarify and confirm issues central to their needs by allowing the ubiquitous role of automated sales technologies, interactive tools, and business intelligence (Sheth and Sharma 2008). However, Thaichon et al. (2018) also does not consider ISR as an independent sales group as like OSR by describing that customers proceed through different components of the hybrid sales structure (e.g., online) to make a purchase. All other researches also consider ISR only as traditional middleman supporting information to consumers (e.g., Rutherford et al. (2014)), building customer relationship before handing over to OSR (e.g., Kozlenkova et al. (2017), Thaichon et al. (2018)), or preparing for emergency such as online channels break down (e.g., Arli et al. (2018)).

However, non-academic articles from the industry have been reporting that ISR's role is evolving, size of ISR is increasing, and so ISR should receive sales incentive as like OSR. Krogue (2013) defines that ISR is a professional sales job remotely with multiple touchpoints to create a sales close and involving medium or large ticket goods and services in B2B or high-end B2C transactions. Furthermore, Krogue (2013) differentiates ISR from telemarketing (e.g., a scripted, single-call-close, almost targeting a small-ticket), customer service, and inbound call centers. Zoltners et al. (2013) and Green (2013a,b) also argue that ISR's size in B2B is increasing for three reasons; firms' cost efficiency pressure, buyers' online purchasing availability, and relationship building availability using online tools (e.g., webinar and videoconferencing). Finally, ZSAssociates and RealityWorks (2014) show ISRs' many of compensation issues such as quota setting fairness, the competitiveness of total pay levels with the market, incentive plan complexity, or incentive plan not motivational enough. These survey findings imply that ISRs also receive incentives as like OSR in the B2B industry, and the firms strive to find out a sound incentive system for ISR.

Hence, there is a gap between modern industry and academic research regarding the two independent sales force groups of ISR and OSR. As far as researchers maintain the traditional perspective considering ISR as a support department for OSR, 
they cannot ask the right question nor provide proper guidance to firms. For example, how to motivate ISR and OSR to improve sales performance independently and to encourage cooperating for overall firm's profit? As an independent sales force, ISR will build a relationship by qualifying potential leads and close the deal as like OSR. Accordingly, for enhancing a firm's total profit, both ISR and OSR should increase their sales performance independently. Moreover, to reduce the loss of potential consumers due to noncooperative multi-sales forces, firms need to promote their cooperating. In the initial purchasing journey, B2B buyers may not know clearly what product and solution are feasible for their firm's issue and so initial contact point may need to be switched from OSR to ISR or vise versa. Yet, if ISR and OSR are in a competitive relationship within the firm, the potential customer may not receive proper service and end up leaving the firm. Sequentially, one of the solutions to encourage them cooperating is providing incentives on cooperative outcomes.

Incentivizing or structuring multiple people as a team in other contexts to promote cooperating and increase benefit is not a new solution. Caldieraro and Coughlan (2009) find that compensation with a team incentive increases the firm's profit when team members are diversified in different territories. Lim and Chen (2014) also find three conditions that team incentive produces better outcomes. Many companies already structure their sales forces in groups or teams based on geographic districts, products categories, or customer segments (Schmitz 2013) and provides team incentives (Caldieraro and Coughlan 2009). Many researchers argue that team-based selling models lead to positive team outcomes, team performance, firms coordinated strategy, cross-selling goal, and customer relationship improvement (Moon and Armstrong 1994, Mohammed and Dumville 2001, Moorman and Albrecht 2008, Ahearne et al. 2010a, Schmitz 2013, Rapp et al. 2014). It is because a sense of shared commitment, synergy among team members, and performance evaluation from customers or coworkers will cause teams' outcome increase (Guzzo and Dickson 1996). Hence, 
Dixon et al. (2002) suggest that managers should control salespeople by monitoring sales team members responses to conflict, encourage internal cognitive voice behaviors, and intervene in teams for the proper function of team selling. However, there are two problems to adapt to the group selling structure and incentives for ISR and OSR. First, ISR and OSR are independent sales teams dealing with different consumers. Second, there is debate regarding behavior-based control, which addresses the process of selling rather than merely the outcomes (Anderson and Oliver 1987).

Behavior-based control systems affect salespeoples efforts by being evaluated and compensated on product knowledge, presentation quality, closing ability, services performed, several active accounts, calls made, and amount of correspondence and days worked (Jackson Jr et al. 1983, Anderson and Oliver 1987). Anderson and Oliver (1987) also propose that behavior-based control system leads to salespeople increasing product and company knowledge, commitment to the sales organization, intrinsic motivation, and using customer-oriented strategies (Saxe and Weitz 1982). Although behavior-based control system sounds ideal solution to motivate reps to behave as firm's goal, difficulty of measuring reps' effort and monitoring costs obstruct its application. As an alternative, outcome-based control system, using sales volume (e.g., in dollars or number of unites), is more applicable than the behavior-based control (Churchill Jr et al. 1985). However, outcome-based control may harm the firms in the long run because salespeople will neglect extra effort in selling new products, prospecting, or customer satisfaction (Anderson and Oliver 1987).

Consequently, compensation utilizing both control systems will be a better solution to enhance both sales and cooperative outcomes in ISR and OSR's independent sales team context. For instance, Hauser et al. (1996) investigate a target-value compensation system which integrates mutual evaluation policy with outcome-based compensation plan when the R\&D and marketing teams at a global consumer products company need to cooperate. They found that the suggested target-value compensa- 
tion system using peer evaluation can induce optimal agents efforts and maximize the firm's profit. In Hauser et al. (1996), the combination of behavior-based and outcome-based control compensation scheme remedies each system's shortcomings. Krafft et al. (2012) also show the positive results that compensation and behavior control systems can affect salespersons behaviors and outcomes.

In this paper, therefore, I allow the new ISR's independent selling role required from the consumers' behavior actively using online communication, and so, I consider that ISR and OSR may compete to increase own sales outcomes. Besides, when new B2B consumers do not have a clear clue, they need consulting from salespeople. If the initial contact salesperson qualifies potential's needs well and hands over to the right salesperson for the consumers' benefit, it will also lead to the firm's overall gain. However, under the ISR and OSR's competitive environment, sales reps are not likely to behave for potential consumers and the firm's benefits. Hence, I adapt the targetvalue compensation plan for ISR and OSR to motivate them enhancing both their independent sales performance and cooperative sales outcomes from Hauser et al. (1996).

\section{Target-Value Compensation Plan}

I consider a firm with two different sales reps: an Inside Sales Rep (ISR) and an Outside Sales Rep (OSR). To develop my model predicting ISR's and OSR's cooperation under a target-value compensation plan, I introduce the following assumptions about reps and the firm. Standard assumptions from previous agency-theoretic sales force compensation models are described from (a) - (e). Table 7 encapsulates parameters' notation used in the model.

a) I assume a linear sales response function, i.e., $\hat{S}=h+\beta e+\varepsilon, \varepsilon \sim \mathcal{N}\left(0, \sigma^{2}\right)$ Holmstrom and Milgrom (1987), Lal and Srinivasan (1993), Misra et al. (2005), Daljord 
Table 7: List of Symbols

\begin{tabular}{ll}
\hline \hline Symbols & Definitions \\
\hline $\mathrm{i}$ & Inside Sales Rep \\
$\mathrm{o}$ & Outside Sales Rep \\
$\mathrm{S}$ & Total Sales \\
$w$ & Total compensation for ISR (Inside Rep) \\
$v$ & Total compensation for OSR (Outside Rep) \\
$w_{0}, v_{0}$ & Fixed salary \\
$w_{1}, v_{1}$ & Commission rate on sales \\
$w_{2}, v_{2}$ & Commission rate on sales \\
$w_{3}, v_{3}$ & Commission rate on handed over leads \\
$w_{4}, v_{4}$ & Penalty rate on discrepancy of reported and actually handed over leads \\
$\mu, \rho$ & Risk-aversion of the ISR and OSR respectively \\
$e_{i i s}, e_{o o s}$ & ISR's (OSR's) qualifying, selling, and closing effort \\
$e_{i q}, e_{o q}$ & ISR's (OSR's) qualifying effort to hand over to OSR (ISR) \\
$e_{i o s}, e_{o i s}$ & ISR's (OSR's) closing effort on handed over leads from OSR (ISR) \\
$\beta_{i i s}, \beta_{o o s}$ & ISR's (OSR's) marginal selling productivity on own leads \\
$\beta_{i q}, \beta_{o q}$ & ISR's (OSR's) marginal qualifying productivity for handing over to the other side \\
$\beta_{i o s}, \beta_{o i s}$ & ISR's (OSR's) marginal selling productivity on the other leads from the other side \\
$p_{i}, p_{o}$ & ISR's (OSR's) selling product'price \\
$c_{i i s}, c_{o o s}$ & Cost of selling effort on own leads \\
$c_{i q}, c_{o q}$ & Cost of qualifying effort for handing over to the other side \\
$c_{i o s}, c_{o i s}$ & Cost of qualifying effort on the other leads from the other side \\
$\sigma_{i 1}^{2}\left(\sigma_{o 1}^{2}\right)$ & Variance of market uncertainty when ISR (OSR) sells products from her/his own leads \\
$\sigma_{i 2}^{2}\left(\sigma_{o 2}^{2}\right)$ & Variance of market uncertainty when ISR (OSR) sells products from OSR's (ISR's) leads \\
$U$ & Net utility by salesperson \\
$\bar{F}$ & Reservation wage \\
$\pi$ & Firms' total net profits from ISR and OSR \\
$\epsilon$ & Market uncertainty \\
\hline \hline
\end{tabular}

et al. (2016). $h$ represents base level of sales of her territory because of carryover and firms' other marketing efforts, $e \in \mathbb{Z}^{+}$denotes selling effort, $\beta \in \mathbb{R}^{+}$is the agent's margin productivity, and $\varepsilon$ is the random disturbance term, normally distributed with zero mean and variance $\sigma^{2}$.

b) A salesperson maximizes her/his expected net utility $\left(U_{i}, U_{o}\right)$ which consists of his/her income and disutility. Technically, let $u_{i}(w)$ denote IRS's utility function for her income $w$, and $u_{o}(v)$ denote ORS's utility function for his income $v \cdot c_{i}(e)\left(c_{o}(e)\right)$ denotes ISR's (OSR's) disutility associated with efforts $e$. Overall, ISR's (OSR's) 'net utility' is represented as $U_{i}=f\left(u_{i}(w)-c_{i}(e)\right)\left(U_{o}=f\left(u_{o}(w)-c_{o}(e)\right)\right)$.

c) I assume that reps are risk averse, and so rep's marginal utility of income is diminishing, i.e., $u_{i}(w) \geqq 0, u_{i}^{\prime}(w)>0, u_{i}^{\prime \prime}(w)<0$. More specifically, I assume salespeople's utility function of income can be specified as constant absolute risk aversion functions, i.e., $u_{i}(w)=-\exp \left(-\mu\left(w\left(s_{i}\right)\right)\right)\left(u_{o}(v)=-\exp \left(-\rho\left(v\left(s_{o}\right)\right)\right)\right)$ where the absolute risk aversion parameters are assumed to be positive $(\mu, \rho>0)$.

d) The sales rep's disutility is an increasing function of selling effort, i.e., $c(e) \geqq 0$, $c^{\prime}(e)>0, c^{\prime \prime}(e)>0$.

e) (1) The firm's marginal cost of production of the product or service and gross 
margin per unit are constant. (2) The firm is risk neutral and has the objective of maximizing its expected profits $E[\pi]$. (3) The sales rep's and the firm's information about the form and parameters of the sales rep's sales response function and utility function is symmetric.

Next are my non-standard model assumptions:

f) When there are two sides of reps (ISR, OSR), firm's total sales function consists of each rep's sales function, e.g., $\hat{S}=\hat{S}_{i}+\hat{S}_{o}$, where $\hat{S}_{i}$ is the ISR's sales outcome and $\hat{S}_{o}$ is the OSR's. Moreover, I divide each rep's sales function into three compositions when ISR and OSR exchange their qualified leads as below.

$$
\begin{gathered}
\hat{S}_{i}=h_{i}+\hat{S}_{i 1}+\hat{S}_{i 2} \\
\hat{S}_{o}=h_{o}+\hat{S}_{o 1}+\hat{S}_{o 2}
\end{gathered}
$$

Specifically, ISR's sales $\left(\hat{S}_{i}\right)$ consists of the base level of sale $\left(h_{i}\right)$, ISR's plain sales $\left(\hat{S}_{i 1}\right)$, and ISR's sales based on OSR's cooperation $\left(\hat{S}_{i 2}\right)$ (equation $3.1(\mathrm{~b})$ ). Similarly, (equation $3.1(\mathrm{c})$ ) is OSR's sales function.

g) Each rep's plain sales $\left(\hat{S}_{i 1}, \hat{S}_{o 1}\right)$ comes from her/his own effort and marginal productivity. Importantly, when a rep achieves sales from the other side's well qualified leads, its sales functions $\left(\hat{S}_{i 2}, \hat{S}_{o 2}\right)$ imply that both reps' efforts and marginal productivity are multiplicative.

$$
\begin{gathered}
\hat{S}_{i}\left\{\begin{array}{l}
\hat{S}_{i 1}=p_{i} \beta_{i i s} e_{i i s}+\varepsilon_{i 1}, \varepsilon_{i 1} \sim \mathcal{N}\left(0, \sigma_{i 1}^{2}\right) \\
\hat{S}_{i 2}=p_{i} \beta_{i o s} e_{i o s} \beta_{o q} e_{o q}+\varepsilon_{i 2}, \varepsilon_{i 2} \sim \mathcal{N}\left(0, \sigma_{i 2}^{2}\right)
\end{array}\right. \\
\hat{S}_{o}\left\{\begin{array}{l}
\hat{S}_{o 1}=p_{o} \beta_{o o s} e_{o o s}+\varepsilon_{o 1}, \varepsilon_{o 1} \sim \mathcal{N}\left(0, \sigma_{o 1}^{2}\right) \\
\hat{S}_{o 2}=p_{o} \beta_{o i s} e_{o i s} \beta_{i q} e_{i q}+\varepsilon_{o 2}, \varepsilon_{o 2} \sim \mathcal{N}\left(0, \sigma_{o 2}^{2}\right)
\end{array}\right.
\end{gathered}
$$

where $\forall e>0\left(e \in \mathbb{R}^{+}\right)$are reps' efforts, $\forall \beta>0\left(\beta \in \mathbb{R}^{+}\right)$are the marginal productiv- 
ity of rep's effort, $\forall p>0\left(p \in \mathbb{R}^{+}\right)$are products' prices, and $\varepsilon$ is normally distributed market uncertainty (table 7).

h) Sales reps have convex disutility (cost) function associated with three different leads' resources; the effort of qualifying and selling to own leads, qualifying leads effort to hand over to the other side and selling effort for qualified leads from the other. More specifically, I posit the total cost of working efforts is an additively separable function of the disutility, and basic selling effort: i.e., $c_{i}\left(e_{i i s}, e_{i q}, e_{i o s}\right)=\frac{c_{i i s} e_{i i s}^{2}}{2}+\frac{c_{i q} e_{i q}^{2}}{2}+\frac{c_{i o s} e_{i o s}^{2}}{2}$, where $c_{i i s}>0, c_{i q}>0$, and $c_{i o s}>0$ are the respective cost parameters. It is because each effort procedure is separable. For example, rep's pure selling effort $\left(e_{i i s}\right)$ implies that the rep qualified potentials, met the customer and sold the product from his/her own leads. Rep's qualifying effort for the other side $\left(e_{i q}\right)$ implies that the rep recognized some of his potentials need the other side's service and guidance, qualified these potentials, and handed over to the other rep. Finally, rep's selling effort from the other rep $\left(e_{i o s}\right)$ implies that the rep received new potentials from the other side, learned the potentials' needs, and sold the product.

\section{Wage Target Value Function}

In this paper, I will adopt the wage target value function as a compensation scheme to encourage reps to exchange potentials with preventing morale hazard such as exchanging unqualified leads (Hauser et al. 1996). Under a standard compensation, salespeople receive a salary plus incentive (or bonus) on sales to motivate salespeople increasing sales outcome. Accordingly, firms can incentivize reps' cooperative outcome using an incentive system on collaborative results. More specifically, a firm may want to encourage ISR, and OSR exchanging well-qualified potential leads if they find that their potential consumers need the other side's service and products. Since handing over these potential consumers to the others requires extra effort (e.g., qualifying the potentials, delivering information to the other side), the rep may leave 
the potential for other consumers or try to sell him/herself. Either way, it will damage a firm's potential profits. The better way to the potential consumers is that reps hand over the potential to the right salesperson.

$$
\begin{gathered}
I S R: w\left(\hat{s}_{i 1}, \hat{s}_{i 2}, \hat{k}_{i o}, \hat{q}_{i}\right)=w_{0}+w_{1} \hat{s}_{i 1}+w_{2} \hat{s}_{i 2}+w_{3} k_{i o}-w_{4}\left(\hat{s}_{o 2}-\hat{q}_{i}\right)^{2} \\
\text { OSR }: v\left(\hat{s}_{o 1}, \hat{s}_{o 2}, \hat{k}_{o i}, \hat{q}_{o}\right)=v_{0}+v_{1} \hat{s}_{o 1}+v_{2} \hat{s}_{o 2}+v_{3} k_{o i}-v_{4}\left(\hat{s}_{i 2}-\hat{q}_{o}\right)^{2}
\end{gathered}
$$

where $w_{0}, v_{0}$ are basic salary terms, $w_{1}, v_{1}$ show incentives on each rep's own sales outcome, $w_{2}, v_{2}$ represent incentives on each rep' sales outcome from the other rep's qualified leads, $w_{3}, v_{3}$ mean incentive on the recorded number of leads ISR (OSR) gave to OSR (ISR), and $w_{4}, v_{4}$ imply the forecasted value of leads which ISR (OSR) promises to give to OSR (ISR).

I specify the recorded number of leads which reps gave to the other side $\left(\hat{k}_{i o}, \hat{k}_{o i}\right)$ as;

$$
\begin{aligned}
& k_{i o}=\beta_{i q} e_{i q}+\Omega_{i}, \\
& k_{o i}=\beta_{o q} e_{o q}+\Omega_{o}
\end{aligned}
$$

where $e_{i q}\left(e_{o q}\right)$ is ISR(OSR)'s qualifying effort to hand over, $\beta_{i q}\left(\beta_{o q}\right)$ is ISR(OSR)'s marginal productivity, and $\Omega_{i}\left(\Omega_{o}\right)$ is deceitfully recorded number of leads which ISR (OSR) handed over to OSR (ISR). Hence, the portion of $\beta_{i q} e_{i q}$ out of total number of recorded leads $\left(k_{i o}\right)$ is well-qualified by ISR. However, $\Omega_{i}$ is the portion of leads that ISR handed over to OSR to gain incentive $w_{3}$ without effort $\left(e_{i q}\right)$. If the firm offers incentives $w_{3}, v_{3}$ based on recorded number of exchanged leads, reps' faked number of exchanged leads $\left(\Omega_{i}, \Omega_{0}\right)$ will increase without limit and firm's profit will decrease. So, to prevent this moral hazard, I want to invite a punishment on the reps' moral hazard by introducing reps' promise. 
I specify the forecasted value of leads which reps promises to give $\left(q_{i o}, q_{o i}\right)$ as;

$$
\begin{aligned}
& q_{i o}=p_{o}\left(\beta_{i q} e_{i q} \beta_{o i s} e_{o i s}+\Omega_{i}\right), \\
& q_{o i}=p_{i}\left(\beta_{o q} e_{o q} \beta_{i o s} e_{i o s}+\Omega_{o}\right)
\end{aligned}
$$

where $e_{\text {ois }}\left(e_{i o s}\right)$ is ISR(OSR)'s selling effort on the handed over leads from OSR(ISR), $\beta_{\text {ois }}\left(\beta_{\text {ios }}\right)$ is ISR(OSR)'s selling effort' marginal productivity. ISR (OSR) will obtain an insight how much a potential would purchase the firm's products during the qualifying process if ISR(OSR) put proper effort $e_{i q}\left(e_{o q}\right)$. Also, ISR(OSR) would get how much seriously OSR(ISR) take the handed over leads and put selling effort $e_{\text {ois }}\left(e_{\text {ios }}\right)$ when ISR(OSR) informed OSR(ISR) about the potentials. If ISR and OSR communicate properly about potentials, they can forecast closely to $p_{o}\left(\beta_{i q} e_{i q} \beta_{o i s} e_{o i s}\right)$ and $p_{i}\left(\beta_{o q} e_{o q} \beta_{i o s} e_{i o s}\right)$.

That is, if ISR (OSR) hands over purely well-qualified leads, the deceitful record $\Omega_{i}\left(\Omega_{o}\right)$ should be zero. Also, the recorded number of exchanged leads will be equal to reps' actual effort level $\left.\left(k_{i o}=\beta_{i q} e_{i q}, k_{o i}=\beta_{o q} e_{o q}\right)\right)$ in Equation 3.4), and the forecasted value of leads will be similar to reps' cooperating effort level (Equation 3.5).

Note that the boundary number of leads has limit.

$$
0 \leq \Omega_{o} \lessgtr k_{i o} \leq \bar{k}_{i o}
$$

where $\bar{k}_{i o}$ is the initial unqualified leads and all are natural positive numbers $\left(k_{i o}, \bar{k}_{i o}, \Omega_{i} \in\right.$ $\mathbb{N})$.

\section{Firm's Profit Maximization}

Based on the Stackelberg problem, I assume the firm maximizes firm's expected profit to set the optimal incentive plans based on ISR and OSR's condition such that they should receive a minimum wage to stay in the firm (Individual Rationality) and they 
will choose the best effort level to maximize their utility (Incentive Compatibility).

(M1)

$$
\max _{w, v \in \mathbb{R}^{+}} \mathrm{E}\left[\hat{s}_{i}+\hat{s}_{o}-\hat{w}-\hat{v}\right]
$$

s.t:

$$
\begin{gathered}
\text { (IR) ISR: } \mathrm{E}\left[U_{i}\left(\hat{w}_{i}-c_{i}\right)\right] \geq \bar{F}_{i}, \\
\text { OSR: } \mathrm{E}\left[U_{o}\left(\hat{w}_{o}-c_{o}\right)\right] \geq \bar{F}_{o} \\
(\mathrm{IC}) \operatorname{ISR}: e(w), \Omega_{i}(w) \in \underset{e, q_{i} \in \mathbb{N}}{\arg \max } \mathrm{E}\left[U\left(w-D\left(e, q_{i}\right)\right)\right], \\
\text { OSR: } e(v), \Omega_{o}(v) \in \underset{e, q_{o} \in \mathbb{N}}{\arg \max } \mathrm{E}\left[U\left(v-D\left(e, q_{o}\right)\right)\right]
\end{gathered}
$$

From the IC constraint (Equation 3.8), I can obtain salespeople's reaction to the incentives. Table 8 shows ISR and OSR's choice regarding incentives. As Lal and Srinivasan (1993) have shown that reps' selling effort will increase with an increase of sales incentive under a standard compensation plan (salary plus incentive on sales), my result also shows that both reps' selling efforts $\left(e_{i i s}, e_{o o s}\right)$ on own leads depend on only sales incentive $\left(w_{1}, v_{1}\right)$. When the sales incentive increases, the reps' selling effort will also increase. Also, rep's cooperating effort $\left(e_{i q}, e_{o q}\right)$ which qualifying leads to hand over to the other side will increase when the incentive on the delivered number of leads $\left(w_{3}, v_{3}\right)$ increases. The expected results are consistent with previous literature and confirm that the model is considerable. Then, I found interesting new insights of how salespeople will change their effort strategy based on cooperative incentive and penalty on dishonest as below.

Proposition 8. Rep's Strategy based on Target Value Wage

(a) When $O S R(I S R)$ 's cooperative qualifying effort incentive increases $\left(v_{3}\left(w_{3}\right)\right)$, ISR $(O S R)$ 's selling effort on the delivered leads from the other side $\left(e_{i o s}\left(e_{o i s}\right)\right)$ will increase.

(b) When ISR(OSR)'s penalty on dishonest increases $\left(w_{4}\left(v_{4}\right)\right)$, ISR $(O S R)$ will reduce 
Table 8: Salespeople's Choices Associated with Incentives

\begin{tabular}{lrlr}
\hline \hline & ISR's optimal Decision & & OSR's optimal Decesion \\
\hline$e_{i i s}$ & $\frac{w_{1} p_{i} \beta_{i i s}}{c_{i i s}}$ & $e_{o o s}$ & $\frac{v_{1} p_{o} \beta_{o o s}}{c_{o o s}}$ \\
$e_{i o s}$ & $\frac{w_{2} v_{3} \beta_{i o s} \beta_{o q}^{2} p_{i}^{2}}{c_{i o s} c_{o q}}$ & $e_{o i s}$ & $\frac{v_{2} w_{3} \beta_{o i s} \beta_{i q}^{2} p_{o}^{2}}{c_{o i s} c_{i q}}$ \\
$e_{i q}$ & $\frac{w_{3} p_{o} \beta_{i q}}{c_{i q}}$ & $e_{o q}$ & $\frac{v_{3} p_{i} \beta_{o q}}{c_{o q}}$ \\
$\Omega_{i}$ & $\frac{w_{3}}{p_{o}^{2}}\left(\frac{1}{2 w_{4}}-\mu \sigma_{i 2}^{2}\right)$ & $\Omega_{o}$ & $\frac{v_{3}}{p_{i}^{2}}\left(\frac{1}{2 v_{4}}-\rho \sigma_{o 2}^{2}\right)$ \\
\hline \hline
\end{tabular}

his/her deceitful report portion $\left(\Omega_{i}\left(\Omega_{o}\right)\right)$ but ISR (OSR) will not decrease or increase other selling efforts.

Proof: See appendix 6.2.

Interestingly, proposition 8(a) presents that reps' selling effort on the delivered leads from the other side $\left(e_{i o s}, e_{o i s}\right)$ depend on both of own incentive of the cooperative sales $\left(w_{2}, v_{2}\right)$ and the other's incentive on cooperating effort $\left(w_{3}, v_{3}\right)$ (Table 8$)$. This finding implies that the firm's decision to increase incentive on the exchanging qualified leads has an unexpectedly positive effect on the other side reps' selling effort based on the exchanged leads. Besides, the most exciting finding is that the penalty $\left(w_{4}, v_{4}\right)$ depletes the rep's deceitful report $\left(\Omega_{i}, \Omega_{o}\right)$ and it does not reduce reps' any other efforts (proposition $8(\mathrm{~b}))$. When the incentives $\left(w_{3}, v_{3}\right)$ on the recorded number of leads which reps gave to the other side $\left(\hat{k}_{i o}, \hat{k}_{o i}\right)$ increase, reps' deceitful report of the number of leads increase. This side effect is one of the major reasons why firms do not want to pay incentive on recorded cooperating activity. However, when the penalty scheme only seizes the rep's temptation of fake without reducing reps' other efforts, the compensation strategy becomes successful in encouraging working in the right way. Therefore, I can learn that reps' cooperative qualifying and cooperative selling efforts will increase without damage when the firm uses the target value wage system. 


\section{Optimal Solutions}

Table 9 summarizes the firm's optimal choices and Table 10 represents the ISR and OSR's optimal choices under the target value compensation plan scenario. In these tables, the firm's optimal incentives and reps' optimal effort strategies are complex to capture the effects of reps' margin productivity, effort costs, risk aversion, and market uncertainty. Hence, I will discuss each parameters' effects specifically with the comparative statics table 11, 12. For now, I will focus on two critical points.

Table 9: Optimal Wage Parameters

\begin{tabular}{cccc}
\hline \hline $\mathrm{w}$ & ISR's optimal incentive & $\mathrm{v}$ & OSR's optimal incentive \\
\hline$w_{1}^{*}$ & $\frac{p_{i}^{2} \beta_{i i s}^{2}}{\left(p_{i}^{2} \beta_{i i s}^{2}+2 c_{i i s} \mu \sigma_{i 1}^{2}\right)}$ & $v_{1}^{*}$ & $\frac{p_{o}^{2} \beta_{o o s}^{2}}{\left(p_{o}^{2} \beta_{o o s}^{2}+2 c_{o o s} \rho \sigma_{o 1}^{2}\right)}$ \\
$w_{2}^{*}$ & $\frac{\beta_{i o s} \beta_{o q}-\sqrt{\beta_{i o s}^{2} \beta_{o q}^{2}-c_{i o s} c_{o q}}}{p_{i} \beta_{i o s} \beta_{o q}}$ & $v_{2}^{*}$ & $\frac{\beta_{o i s} \beta_{i q}-\sqrt{\beta_{o i s}^{2} \beta_{i q}^{2}-c_{o i s} c_{i q}}}{p_{o} \beta_{o i s} \beta_{i q}}$ \\
$w_{3}^{*}$ & $\frac{c_{i q} \sigma_{o 2}}{p_{o}^{2} \beta_{i q}^{2} \beta_{o i s}} \sqrt{\frac{2 c_{o i s} \rho\left(\beta_{o i s} \beta_{i q}-\sqrt{\left.\beta_{o i s}^{2} \beta_{i q}^{2}-c_{o i s} c_{i q}\right)}\right.}{\sqrt{\beta_{o i s}^{2} \beta_{i q}^{2}-c_{o i s} c_{i q}}}}$ & $v_{3}^{*}$ & $\frac{c_{o q} \sigma_{i 2}}{p_{i}^{2} \beta_{o q}^{2} \beta_{i o s}} \sqrt{\frac{2 c_{i o s} \mu\left(\beta_{i o s} \beta_{o q}-\sqrt{\left.\beta_{i o s}^{2} \beta_{o q}^{2}-c_{i o s} c_{o q}\right)}\right.}{\sqrt{\beta_{i o s}^{2} \beta_{o q}^{2}-c_{i o s} c_{o q}}}}$ \\
$w_{4}^{*}$ & $\frac{1}{2 \mu \sigma_{i 2}^{2}}$ & $v_{4}^{*}$ & $\frac{1}{2 \rho \sigma_{o 2}^{2}}$ \\
\hline \hline
\end{tabular}

Table 10: Salespeople's Optimal Choices

\begin{tabular}{crrr}
\hline \hline & ISR's optimal Decision & & OSR's optimal Decision \\
\hline$e_{i i s}^{*}$ & $\frac{p_{i}^{3} \beta_{i i s}^{3}}{c_{i i s}\left(p_{i}^{2} \beta_{i i s}^{2}+2 c_{i i s} \mu \sigma_{i 1}^{2}\right)}$ & $e_{o o s}^{*}$ & $\frac{p_{o}^{3} \beta_{o s}^{3}}{c_{o o s}\left(p_{o}^{2} \beta_{o o s}^{2}+2 c_{o o s} \rho \sigma_{o 1}^{2}\right)}$ \\
$e_{i o s}^{*}$ & $\frac{\sigma_{i 2}\left(\beta_{i o s} \beta_{o q}-\sqrt{\beta_{i o s}^{2} \beta_{o q}^{2}-c_{i o s} c_{o q}}\right)}{p_{i} \beta_{i o s} \beta_{o q} c_{i o s}} \hat{x}$ & $e_{o i s}^{*}$ & $\frac{\sigma_{o 2}\left(\beta_{o i s} \beta_{i q}-\sqrt{\beta_{o i s}^{2} \beta_{i q}^{2}-c_{o i s} c_{i q}}\right)}{p_{o} \beta_{o i s} \beta_{i q} c_{o i s}} \hat{y}$ \\
$e_{i q}^{*}$ & $\frac{\sigma_{o s}}{p_{o} \beta_{i q} \beta_{o i s}} \hat{y}$ & $e_{o q}^{*}$ & $\frac{\sigma_{i 2}}{p_{i} \beta_{o q} \beta_{i o s}}$ \\
$\Omega_{i}^{*}$ & $\frac{c_{i q} \sigma_{o 2}}{p_{o}^{4} \beta_{i q}^{2} \beta_{o i s}}\left(\mu \sigma_{i 2}^{2}-\mu \sigma_{i 2}^{2}\right)=0$ & $\Omega_{o}^{*}$ & $\frac{c_{o q} \sigma_{i 2}}{p_{i}^{4} \beta_{o q}^{2} \beta_{i o s}}\left(\rho \sigma_{o 2}^{2}-\rho \sigma_{o 2}^{2}\right)=0$ \\
\hline \hline & note: $\hat{x}=\sqrt{\frac{2 c_{i o s} \mu\left(\beta_{i o s} \beta_{o q}-\sqrt{\left.\beta_{i o s}^{2} \beta_{o q}^{2}-c_{i o s} c_{o q}\right)}\right.}{\sqrt{\beta_{i o s}^{2} \beta_{o q}^{2}-c_{i o s} c_{o q}}}}, \hat{y}=\sqrt{\frac{2 c_{o i s} \rho\left(\beta_{o i s} \beta_{i q}-\sqrt{\beta_{o i s}^{2} \beta_{i q}^{2}-c_{o i s} c_{i q}}\right)}{\sqrt{\beta_{o i s}^{2} \beta_{i q}^{2}-c_{o i s} c_{i q}}}}$
\end{tabular}

Proposition 9. (a) ISR(OSR)'s optimal incentive on cooperative sales $\left(w_{2}^{*}\left(v_{2}^{*}\right)\right)$ do not depend on any reps' risk aversion $(\mu, \rho)$ and uncertainty $\left(\sigma_{i 2}^{2}, \sigma_{o 2}^{2}\right)$. Instead, (b) OSR(ISR)'s optimal incentive on qualifying leads $\left(v_{3}^{*}\left(w_{3}^{*}\right)\right)$ undertakes ISR $(O S R)$ 's risk aversion $(\mu(\rho))$ and uncertainty $\left(\sigma_{i 2}^{2}\left(\sigma_{o 2}^{2}\right)\right)$. 
Proof: See appendix 6.2.

Proposition 9 shows an exciting implication that qualifying process is responsible for reps' risk aversion and market uncertainty. Although I do not explicitly divide the selling process into qualifying and selling stages in this paper, the two-side reps' cooperative efforts intimate there are two different stages. More specifically, when ISR qualifies potentials to hand over, the effort of qualifying $\left(e_{i q}\right)$ does not include any selling process. When OSR receives the qualified potentials from ISR, the only OSR has to proceed is selling products $\left(e_{o i s}\right)$. Sequentially, the commission on the qualifying to hand over the potentials $\left(w_{3}^{*}\right)$ is incentivizing qualifying efforts and the commission on the selling $\left(v_{2}^{*}\right)$ is incentivizing selling process. If the incentive on the selling process increases when risk aversions and market uncertainties increase, reps are taking a risk on the market uncertainty during the selling procedure. However, Proposition 9 (a),(b) (Table 9) show that the only incentive on qualifying process increases when risk aversions and market uncertainties increase. Hence, I can conclude that firms compensate reps' risk aversion and uncertainty in the qualifying stage.

Proposition 10. Penalty at the Optimal Level

(a) The firm should increase the optimal penalty of the dishonest report $\left(w_{4}^{*}, v_{4}^{*}\right)$ when reps' risk aversion $(\mu, \rho)$ or uncertainty $\left(\sigma_{i 2}^{2}, \sigma_{o 2}^{2}\right)$ decreases.

(b) Under the optimal penalty level, the reps' optimal choices of the dishonest report $\left(\Omega_{i}^{*}, \Omega_{o}^{*}\right)$ are zero regardless any other parameters' increase.

Proof: See appendix 6.2.

Proposition 10 predicts the ideal results for managers and firms. Proposition 10(a) shows that the firm's optimal penalty negatively depends on the rep's risk aversion and uncertainty. For example, when reps are more risk-takers (e.g., low-risk aversion), reps will try to increase the deceitful report of qualified leads. Hence, the firm should increase the penalty rate to prevent the high number of fake reports. When market uncertainty is low, reps will increase the fake report. It is because low 
market uncertainty implies the market demand is high, and so, high selling effort's marginal productivity is expected. In the stable market, reps will not try to put more qualifying effort to target high potentials. Thus, the firm should increase the penalty to foil reps' deceit on exchanging a number of qualified leads. Further, Proposition 10(b) dispels the concern about reps' false reports at the optimal level, because reps' optimal dishonest report is zero regardless of any rate of reps' marginal productivity, effort cost, or prices. It implies that if the firm gives activity incentive $\left(w_{3}^{*}, v_{3}^{*}\right)$ on the number of qualified leads that ISR(OSR) gave to OSR(ISR) with the optimal penalty rate, the firm can encourage reps to increase cooperation without overpayment problem on the deceitful number of leads.

Table 11 and Table 12 summarize respectively ISR and OSR's comparative statics regarding the increasing effect of each parameters. In these tables, I will discuss how changes in the exogenous parameters of the model such as uncertainty, risk aversion, disutility parameters, the marginal productivity of effort, and prices impact the endogenous outputs of my model solution under the Target-Value wage. Most of these results are consistent with those of Lal and Srinivasan (1993) (p. 785) derived under the standard compensation plan (salary plus incentive on sales). The derivation of all comparative static results are provided in Appendix ??.

I find two interesting differences in Table 11 and 12 that shows the comparative statics results under the Target-Value wage. First, the increase of uncertainty relevant to cooperation $\left(\sigma_{i 2}^{2}, \sigma_{o 2}^{2}\right)$ has positive effect on firms' profit because the optimal incentive on exchanged leads $\left(w_{3}, v_{3}\right)$ and rep's cooperative qualifying effort $\left(e_{i q}, e_{o q}\right)$ will increase. Specifically, in Table 11, when OSR's cooperative selling's uncertainty $\left(\sigma_{i 2}^{2}\right)$ increases, the optimal incentive on exchanged leads $\left(w_{3}\right)$ raises up. Accordingly, ISR's optimal qualifying effort for the leads $\left(e_{i q}\right)$ increases. Since ISR put more effort to qualify better leads, her/his forecasted value of leads $\left(q_{i}\right)$ and the number of leads for OSR $\left(k_{i o}\right)$ will also increase. Moreover, if ISR's cooperative selling market un- 
Table 11: Comparative Statics for ISR

\begin{tabular}{|c|c|c|c|c|c|c|c|c|c|c|c|c|c|}
\hline Increasing effect of & $\pi$ & $e_{i i s}$ & $e_{i o s}$ & $e_{i q}$ & $\Omega_{i}$ & $k_{i o}$ & $q_{i}$ & $w_{1}$ & $w_{2}$ & $w_{3}$ & $w_{4}$ & $E[w]$ & $\frac{w_{0}}{E[w]}$ \\
\hline \multicolumn{14}{|l|}{ Uncertainty of } \\
\hline ISR's own selling $\left(\sigma_{i 1}^{2}\right)$ & $-*$ & - & 0 & 0 & 0 & 0 & 0 & - & 0 & 0 & 0 & - & + \\
\hline ISR's other selling $\left(\sigma_{i 2}^{2}\right)$ & + & 0 & 0 & 0 & 0 & 0 & 0 & 0 & 0 & 0 & - & + & - \\
\hline OSR's other selling $\left(\sigma_{o 2}^{2}\right)$ & + & 0 & 0 & + & 0 & + & + & 0 & 0 & + & 0 & + & - \\
\hline \multicolumn{14}{|l|}{ Risk Aversion of } \\
\hline ISR $(\mu)$ & + & - & + & 0 & 0 & 0 & 0 & - & 0 & 0 & - & + & - \\
\hline OSR $(\rho)$ & + & 0 & 0 & + & 0 & + & + & 0 & 0 & + & 0 & + & - \\
\hline \multicolumn{14}{|l|}{ Effort Cost for } \\
\hline Own selling $\left(c_{i i s}\right)$ & - & - & 0 & 0 & 0 & 0 & 0 & - & 0 & 0 & 0 & - & + \\
\hline Cooperative selling $\left(c_{i o s}\right)$ & - & 0 & -+ & 0 & 0 & + & -+ & 0 & + & 0 & 0 & + & - \\
\hline Qualifying for other $\left(c_{i q}\right)$ & - & 0 & 0 & + & 0 & 0 & 0 & 0 & 0 & + & 0 & + & - \\
\hline Cooperative selling $\left(c_{o i s}\right)$ & - & 0 & 0 & + & 0 & 0 & 0 & 0 & 0 & + & 0 & + & - \\
\hline Qualifying for other $\left(c_{o q}\right)$ & - & 0 & + & 0 & 0 & 0 & 0 & 0 & + & 0 & 0 & + & - \\
\hline \multicolumn{14}{|l|}{ Marginal Productivity of } \\
\hline Own selling $\left(\beta_{i i s}\right)$ & + & + & 0 & 0 & 0 & 0 & 0 & + & 0 & 0 & 0 & + & - \\
\hline Cooperative selling $\left(\beta_{i o s}\right)$ & + & 0 & + & 0 & 0 & 0 & 0 & 0 & - & 0 & 0 & - & + \\
\hline Qualifying for other $\left(\beta_{i q}\right)$ & + & 0 & 0 & - & 0 & + & + & 0 & 0 & - & 0 & - & + \\
\hline Cooperative selling $\left(\beta_{\text {ois }}\right)$ & + & 0 & 0 & + & 0 & + & + & 0 & 0 & + & 0 & + & - \\
\hline Qualifying for other $\left(\beta_{o q}\right)$ & + & 0 & + & 0 & 0 & 0 & 0 & 0 & - & 0 & 0 & - & + \\
\hline \multicolumn{14}{|l|}{ Price of } \\
\hline ISR products $\left(p_{i}\right)$ & -+ & + & - & 0 & 0 & 0 & 0 & + & - & 0 & 0 & -+ & -+ \\
\hline OSR products $\left(p_{o}\right)$ & -+ & 0 & 0 & - & 0 & - & - & 0 & 0 & - & 0 & - & + \\
\hline
\end{tabular}

1. + goes up, - goes down, 0 no change.

2. $\pi$ is firms' total profits from both ISR and OSR.

3. * implies that there is a certain condition for the sign.

Table 12: Comparative Statics for OSR

\begin{tabular}{|c|c|c|c|c|c|c|c|c|c|c|c|c|c|}
\hline Effect of & $\pi$ & $e_{o o s}$ & $e_{o i s}$ & $e_{o q}$ & $\Omega_{o}$ & $k_{o i}$ & $q_{o}$ & $v_{1}$ & $v_{2}$ & $v_{3}$ & $v_{4}$ & $E[v]$ & $\frac{v_{0}}{E[v]}$ \\
\hline \multicolumn{14}{|l|}{ Uncertainty of } \\
\hline ISR's other selling $\left(\sigma_{i 2}^{2}\right)$ & + & 0 & 0 & + & 0 & + & + & 0 & 0 & + & 0 & + & - \\
\hline OSR's own selling $\left(\sigma_{o 1}^{2}\right)$ & $-*$ & - & 0 & 0 & 0 & 0 & 0 & - & 0 & 0 & 0 & - & + \\
\hline OSR's other selling $\left(\sigma_{o 2}^{2}\right)$ & + & 0 & 0 & 0 & 0 & 0 & 0 & 0 & 0 & 0 & - & + & - \\
\hline \multicolumn{14}{|l|}{ Risk Aversion of } \\
\hline $\operatorname{ISR}(\mu)$ & + & 0 & 0 & + & 0 & + & + & 0 & 0 & + & 0 & + & - \\
\hline OSR $(\rho)$ & + & - & + & 0 & 0 & 0 & 0 & - & 0 & 0 & - & + & - \\
\hline \multicolumn{14}{|l|}{ Effort Cost for } \\
\hline Own selling $\left(c_{o o s}\right)$ & - & - & 0 & 0 & 0 & 0 & 0 & - & 0 & 0 & 0 & - & + \\
\hline Cooperative selling $\left(c_{\text {ois }}\right)$ & - & 0 & -+ & 0 & 0 & + & -+ & 0 & + & 0 & 0 & + & - \\
\hline Qualifying for other $\left(c_{o q}\right)$ & - & 0 & 0 & + & 0 & 0 & 0 & 0 & 0 & + & 0 & + & - \\
\hline Cooperative selling $\left(c_{\text {ios }}\right)$ & - & 0 & 0 & + & 0 & 0 & 0 & 0 & 0 & + & 0 & + & - \\
\hline Qualifying for other $\left(c_{i q}\right)$ & - & 0 & + & 0 & 0 & 0 & 0 & 0 & + & 0 & 0 & + & - \\
\hline \multicolumn{14}{|l|}{ Marginal Productivity of } \\
\hline Own selling $\left(\beta_{o o s}\right)$ & + & + & 0 & 0 & 0 & 0 & 0 & + & 0 & 0 & 0 & + & - \\
\hline Cooperative selling $\left(\beta_{\text {ois }}\right)$ & + & 0 & + & 0 & 0 & 0 & 0 & 0 & - & 0 & 0 & - & + \\
\hline Qualifying for other $\left(\beta_{o q}\right)$ & + & 0 & 0 & - & 0 & + & + & 0 & 0 & - & 0 & - & + \\
\hline Cooperative selling $\left(\beta_{i o s}\right)$ & + & 0 & 0 & + & 0 & + & + & 0 & 0 & + & 0 & + & - \\
\hline Qualifying for other $\left(\beta_{i q}\right)$ & + & 0 & + & 0 & 0 & 0 & 0 & 0 & - & 0 & 0 & - & + \\
\hline \multicolumn{14}{|l|}{ Price of } \\
\hline ISR products $\left(p_{i}\right)$ & -+ & 0 & 0 & - & 0 & - & - & 0 & 0 & - & 0 & - & + \\
\hline OSR products $\left(p_{o}\right)$ & -+ & + & - & 0 & 0 & 0 & 0 & + & - & 0 & 0 & -+ & -+ \\
\hline
\end{tabular}

1. + goes up, - goes down, 0 no change.

2. $\pi$ is firms' total profits from both ISR and OSR.

3. * implies that there is a certain condition for the sign. 
certainty $\left(\sigma_{i 2}^{2}\right)$ increases, OSR's optimal penalty rate $\left(v_{4}\right)$ decreases (Table 12$)$. This result implies that the Target-Value wage may affect positively to reps to cooperate even when the market circumstance is unstable. As like traditional compensation models, own sales market uncertainties $\left(\sigma_{i 1}^{2}, \sigma_{o 1}^{2}\right)$ reduce reps' optimal selling efforts $\left(e_{i i s}, e_{o o s}\right)$. Hence, the advantage of Target-Value wage is that reps will cooperate more by sharing leads, although the market is in difficulty.

Furthermore, Target-Value wage induces the positive effect of reps' risk aversion $(\mu, \rho)$ on the firm's total profit $(\pi)$. Traditionally, reps' risk aversion affects the firm's profit negatively because the optimal incentive for sales and reps' selling effort will decrease. Table 11, 12 also show that reps' optimal incentive on sales $\left(w_{1}, v_{1}\right)$ and reps' selling effort for own leads $\left(e_{i i s}, e_{o o s}\right)$ decrease. However, under Target-Value, reps' optimal choices among efforts will strategically switch to increase the firm's profit overall. Particularly, when ISR's risk aversion $(\mu)$ increases, OSR's optimal incentive on cooperative leads $\left(v_{3}\right)$ will increase (Table 12). Following that, OSR will increase qualifying effort for ISR $\left(e_{o q}\right)$ and his/her number of leads for ISR $\left(k_{o i}\right)$ and forecasted leads' value $\left(q_{o}\right)$ will increase (Table 12). Sequentially, ISR will increase cooperative selling effort $\left(e_{i o s}\right)$ to achieve OSR's forecasted leads' value (Table 11). For OSR's risk aversion $(\rho)$, vise versa. In result, this chain of cooperative effort choices between the two reps offset the decrease of their own selling effort and induce higher firm's profit.

Proposition 11. New insights of Reps' Cooperative Strategy under the Target-Value Wage

(a) When ISR's cooperative selling marginal productivity $\left(\beta_{\text {ios }}\right)$ increases, ISR's cooperative selling effort $\left(e_{i o s}\right)$ increases. Also, when OSR's cooperative qualifying marginal productivity $\left(\beta_{\text {oq }}\right)$ increases, ISR's cooperative selling effort $\left(e_{i o s}\right)$ increases. For OSR's cooperative selling effort ( $\left.e_{\text {ois }}\right)$, vise versa.

(b) When ISR's cooperative qualifying marginal productivity $\left(\beta_{i q}\right)$ increases, ISR's 
cooperative qualifying effort $\left(e_{i q}\right)$ decreases. However, when OSR's cooperative selling marginal productivity $\left(\beta_{\text {ois }}\right)$ increases, ISR's cooperative qualifying effort $\left(e_{i q}\right)$ increases. For OSR's cooperative qualifying effort $\left(e_{o q}\right)$, vise versa.

Proof: See appendix 6.2.

Proposition 11(a) represents ISR(OSR)'s strategy of cooperative selling effort based on marginal productivity. Precisely, ISR (OSR) will increase cooperative selling effort when OSR (ISR)'s qualifying marginal productivity and selling productivity are high (Table 11 (12)). When OSR (ISR)'s qualifying marginal productivity increases, ISR (OSR) will expect that the leads from OSR (ISR) are highly well qualified and targeted. Hence, ISR (OSR) will likely endeavor to sell products to the potential from OSR (ISR). On the other hand, Proposition 11(b) shows ISR(OSR)'s strategy of cooperative qualifying effort. More specifically, ISR (OSR) will increase cooperative qualifying effort when OSR (ISR)'s cooperative selling productivity. Yet, ISR (OSR) will decrease his cooperative qualifying effort when his/her cooperative qualifying productivity. That is, when ISR and OSR affect each others' sales based on hading over well-qualified leads, they will want to invest their cooperative effort only when the other side has proper ability to qualify leads or sell products well. This finding is a unique contribution representing how ISR and OSR will allocate their efforts when a firm induces reps to cooperate by assigning the Target-Value wage.

Based on all of the result analysis, I can offer two managerial guidance. First, managers can avail themselves of high market uncertainty to raise the firm's profit using Target-Value wage. When one side's market uncertainty increases, the rep will raise cooperative effort and outcomes under the Target-Value wage. When one side's market is in stable condition, the rep will undoubtedly enhance his/her selling effort and outcomes individually. It means that reps will not reduce effort even though one side's market is uncertain, but they will invest the other side of the market to achieve the other incentives. It leads to win-win for both firms and reps, and therefore, the 
Target-Value will be a wiser choice for managers by motivating reps to cooperate and exploit a high potential market. Second, managers should encourage smooth communication between ISR and OSR so that they can recognize and understand each other's cooperative marginal productivity. Because reps will increase only when the other side' collaborative working productivity is high, it is critical that they know each other's productivity. If ISR and OSR understand better each other's productivity and increase cooperative efforts through salespeople's mutual communication, it reduces managers' monitoring costs. Hence, it will be also beneficial to managers and firms.

\section{Extension: Comparison with Alternative Com- pensation Plans}

In this section, I will compare and discuss Target-Value wage's results with alternative plans. The first wage is a standard compensation wage: salary plus incentive on sales which is common in all industries when firms encourage salespeople to enhance sales outcomes. The second one is a combination of incentives on own sales achievement, cooperative sales achievement, and cooperative activity (e.g., multidimensional wage). The multidimensional wage is equal to the Target-Value wage except for no penalty term. It can be applied when firms want to encourage reps to exchange well-qualified leads to magnify firm's overall profit may use the combination of the incentives without penalty.

Equation 5.1 represents the standard compensation scheme and Equation 5.2 represents the multidimensional wage.

$$
\begin{aligned}
\text { Standard Wage } w\left(\hat{s}_{i 1}\right) & =w_{0}+w_{1} \hat{s}_{i 1} \\
v\left(\hat{s}_{o 1}\right) & =v_{0}+v_{1} \hat{s}_{o 1}
\end{aligned}
$$




$$
\begin{array}{r}
\text { Multidimensional Wage } w\left(\hat{s}_{i 1}, \hat{s}_{i 2}, k_{i o}\right)=w_{0}+w_{1} \hat{s}_{i 1}+w_{2} \hat{s}_{i 2}+w_{3} k_{i o} \\
v\left(\hat{s}_{o 1}, \hat{s}_{o 2}, k_{o i}\right)=v_{0}+v_{1} \hat{s}_{o 1}+v_{2} \hat{s}_{o 2}+v_{3} k_{o i}
\end{array}
$$

where $w_{0}, v_{0}$ are basic salary terms, $w_{1}, v_{1}$ show incentives on each rep's own sales outcome, $w_{2}, v_{2}$ represent incentives on each rep' sales outcome from the other rep's qualified leads, and $w_{3}, v_{3}$ mean incentive on the recorded number of leads ISR (OSR) gave to OSR (ISR).

Table 13: Salespeople's Choices Associated with Incentives Under Alternative Plans

\begin{tabular}{lrlrrr}
\hline \hline & \multicolumn{2}{c}{ ISR's Decision } & \multicolumn{2}{c}{ OSR's Decision } \\
\hline \multicolumn{2}{c}{$\begin{array}{c}\text { Standard } \\
\text { Wage }\end{array}$} & Multidimensional & & Standard & Multidimensional \\
\multicolumn{2}{c}{ Wage } & $\frac{w_{1}}{\text { Wage }}$ & Wage \\
\hline$e_{i i s}$ & $\frac{w_{1} p_{i} \beta_{i i s}}{c_{i i s}}$ & $\frac{w_{1} p_{i} \beta_{i i s}}{c_{i i s}}$ & $e_{\text {oos }}$ & $\frac{v_{1} p_{o} \beta_{o o s}}{c_{o o s}}$ & $\frac{v_{1} p_{o} \beta_{o o s}}{c_{o s s}}$ \\
$e_{i o s}$ & 0 & $\frac{w_{2} v_{3} \beta_{i o s} \beta_{o q}^{2} p_{i}^{2}}{c_{i o s} c_{o q}}$ & $e_{o i s}$ & 0 & $\frac{v_{2} w_{3} \beta_{o i s} \beta_{i q}^{2} p_{o}^{2}}{c_{o i s} c_{i q}}$ \\
$e_{i q}$ & 0 & $\frac{w_{3} \beta_{o} \beta_{i q}}{c_{i q}}$ & $e_{o q}$ & 0 & $\frac{v_{3} p_{i} \beta_{o q}}{c_{o q}}$ \\
$\Omega_{i}$ & 0 & $w_{3}$ & $\Omega_{o}$ & 0 & $v_{3}$ \\
\hline \hline
\end{tabular}

Table 14: Optimal Wage Parameters under Alternative Plans

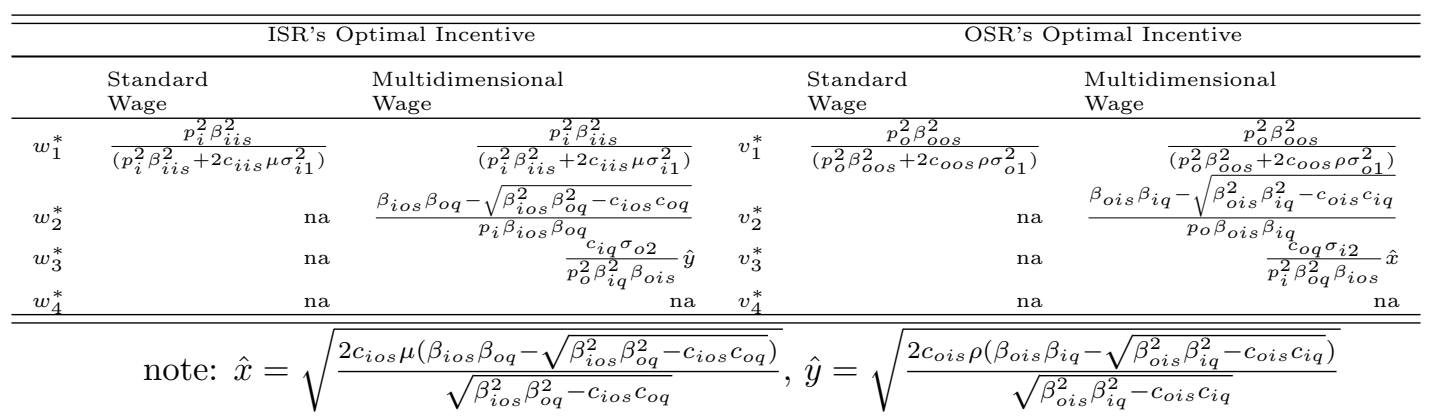

Table 13 shows salespeople's choice and Table 14 represents their optimal incentives under the two alternative plans. By comparing Table 9 and 14, I can confirm that firm's optimal incentives on own sales $\left(w_{1}^{*}, v_{1}^{*}\right)$ are all equal under the three different schemes (e.g., Target-Value, Standard, and Multidimensional wages). Moreover, the optimal incentives $\left(w_{2}^{*}, v_{2}^{*}\right)$ of cooperative sales and $\left(w_{3}^{*}, v_{3}^{*}\right)$ cooperative activities under Target-Value and Multidimensional wages are same. Interestingly, this com- 
parison reveals that the firm's optimal incentive on cooperative sales and cooperative activity is equal regardless of penalty existence in the compensation.

Consequently, both ISR and OSR's responses on incentives under the Target-Value wage and under the other alternative plans are equal (Table 8, 13). Specifically, ISR (OSR) will put the same amount of selling effort on their own leads $\left(e_{i i s}\left(e_{o o s}\right)\right)$ under all three compensation plans. However, both will not put any cooperative selling and qualifying leads effort because of no incentives on the cooperative efforts $\left(k_{i o}, k_{o i}\right)$ or outcomes $\left(s_{i 2}, s_{o 2}\right)$ under the standard wage. Also, under the standard wage, ISR and OSR do not give any deceitful report for the qualified leads to hand over each other because they do not have to hand over any qualified leads under the standard plan. However, it is not good news for firms because ISR and OSR will not focus on solving potential consumers' needs when the other side sales reps are plausible for the potential and then the potential consumers may leave the purchasing process from that firms under firm's standard wage.

More importantly, ISR and OSR will put the same level of cooperative selling and qualifying leads efforts $\left(e_{i o s}, e_{i o s}, e_{i q}, e_{o q}\right)$ under both Target-Value and the multidimensional wages. The only difference is that ISR and OSR's deceitful report of exchanged leads will increase under the multidimensional wage as much as the incentive on the number of leads increases under the multidimensional wage without penalty. That is, the penalty under Target-Value wage has a positive effect of reducing salespeople's deceitful behavior.

\section{Numerical Simulation Analyses}

To check if the closed form of optimal solutions and analyses under the three different wages are consistent, I simply run a numerical simulation. I consider a firm with N number of salespeople, $\mathrm{n}=1, \ldots, \mathrm{N}$ for each IRS and ORS. In this these analyses, I utilize a few values of key parameters of the compensation design problem drown 
from Raju and Srinivasan (1996) for $\mu, \rho$ and ratio between $\beta$, and $c$. Basu and Kalyanaram (1990) conducted experiments to produce these parameters which is also used in Raju and Srinivasan (1996). Table 15 represents the used parameters in the simulation. Since I have new parameters such as $\beta_{i o s}, \beta_{o i s}, \beta_{i q}, \beta_{o q}, p_{i}, p_{o}$, I adjusted the rest of the other parameters for now.

Table 15: Empirical Mean of Parameters for Numerical Simulation

\begin{tabular}{l|r|r|rrr|rrr|rr|r}
\hline \hline & $h$ & $\mu, \rho$ & $\beta_{i i s, o o s}$ & $\beta_{\text {ios }, o i s}$ & $\beta_{i q, o q}$ & $c_{i i s, o o s}$ & $c_{i o s, i o s}$ & $c_{i q, o q}$ & $\sigma_{i 1, o 1}$ & $\sigma_{i 2, o 2}$ & $p_{i, o}$ \\
\hline ISR & 9000 & .3 & .01 & .0099 & .0089 & .03 & .02 & .02 & $1-100$ & $6-105$ & 200 \\
OSR & 10000 & .4 & .01 & .0099 & .0079 & .04 & .03 & .03 & $6-105$ & $11-110$ & 500 \\
\hline \hline
\end{tabular}

Figure 7 shows graphically that the firm's sales and profits and Table 16 represents more specific numerical results (\$) from the firm's outcomes to reps' total expected payoffs. The left three bars in Figure 7 shows that total firm's sales under Target-Value and Multidimensional wages are equal (\$24.8 million) and the total sales under standard wage is lower (\$19.0 million) (first column, Table 16). As I discussed above, the numerical values show that salespeople's own sales are equal under the all three compensation plans ( $\$ 19.0$ million), but ISR and OSR did not produce any cooperative sales based on exchanging qualified leads. That is, expected cooperative sales were $\$ 5.7$ million under Target-Value and multidimensional wages but there is no cooperative sales under the standard wage. It is the reason why firms' total sales under standard wage is lower than the other two wages.

Table 16: Simulation Comparison under the three different plans

\begin{tabular}{lrrlrr}
\hline \hline Wage & $\begin{array}{r}\text { Total } \\
\text { Sales }\end{array}$ & $\begin{array}{r}\text { Cooperative } \\
\text { Sales }\end{array}$ & Own Sales & Firm's & Total \\
& Profit & Reps' Payoff \\
\hline Target Value & $\$ 24,806,929$ & $\$ 5,791,778$ & $\$ 19,015,151$ & $\$ 15,240,022$ & $\$ 9,566,907$ \\
Multidimensional & $\$ 24,806,929$ & $\$ 5,791,778$ & $\$ 19,015,151$ & $\$ 12,748,787$ & $\$ 12,058,142$ \\
Standard & $\$ 19,015,151$ & - & $\$ 19,015,151$ & $\$ 6,617,055$ & $\$ 12,398,096$ \\
\hline \hline
\end{tabular}

Moreover, total reps' payoffs under Target-Value wage is smaller than multidimensional and standard wage. First, when the firm gives the only incentive on sales, 


\section{Firm's Outcomes}

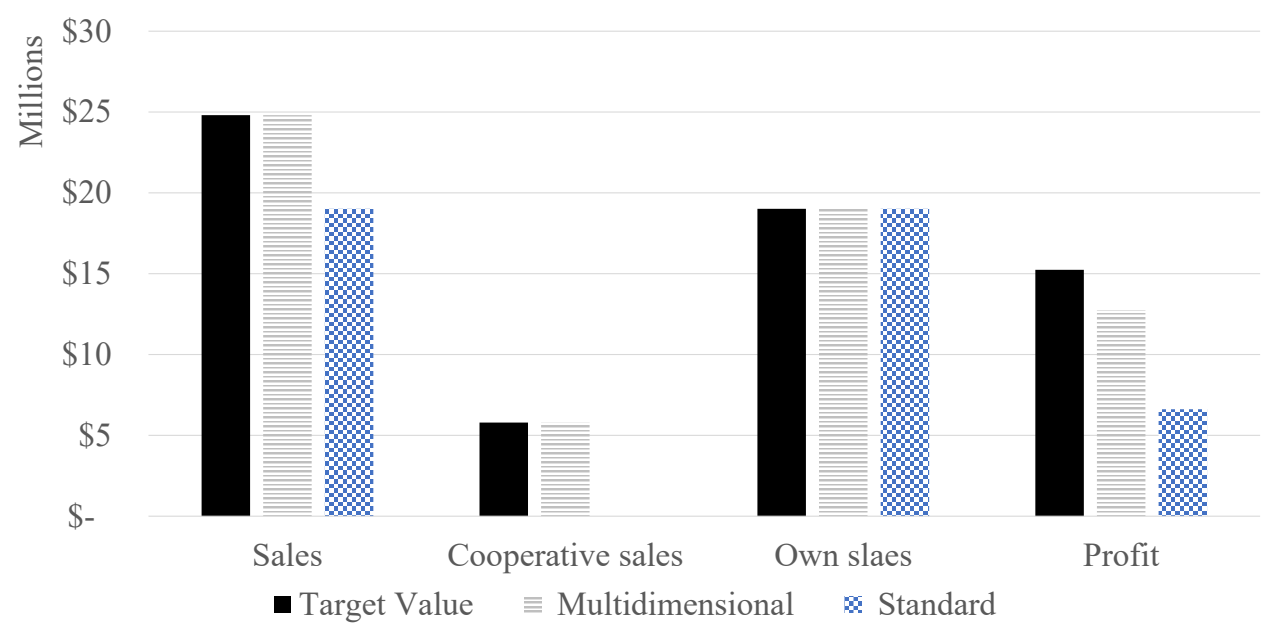

Figure 7: Firm's Profit along with Sales Level

the optimal base salary $(\bar{F})$ is higher than under Target-Value and multidimensional wage. Hence, the overall payment for ISR and OSR under the standard plan is higher than the other two wages. Also, because Target-Value wage includes penalty term, ISR and OSR do not receive extra incentives for deceitful reported leads.

\section{Conclusions and Future Work}

The compensation plan is the biggest standard for sales reps to choose their effort strategy to maximize their utility. Salespeople will focus on sales increase if the compensation plan weight on sales and they will balance their effort between sales and cooperation if the compensation plan includes cooperative activity and sales. Thus, the design of compensation plans that induce the right combination of efforts is a critical issue for sales management. In this paper, I adapt the Target-Value wages encouraging ISR and OSR to cooperate to increase the firm's profit and catch potential consumers. Using my "Target-Value wage," I investigate how ISR and OSR will choose their optimal level of effort. 
In contrast to the standard compensation wage, the Target-Value wage and multidimensional wage proves that the incentive on cooperative qualifying leads motivates ISR and OSR to collaborate by putting qualifying efforts to deliver a good quality of leads. I find that under the Target-Value wage, both reps will give purely wellqualified leads each other because of the dishonest penalty term. However, under the multidimensional wage without penalty, both rep will increase a deceitful number of unqualified leads to receive more reward. Consequently, using numerical simulation, I also support that the firm's profit under Target-Value wage is larger than under the multidimensional wage. Also, I find that the firm should increase the optimal penalty on the deceitful qualified number of leads report when reps are risk-takers or when the market is stable. Since reps will tempt to take any chances to achieve more incentive on deceitful leads under the two conditions, firms' high penalty rate strategy can prevent reps' selfish choice. Moreover, I show that how ISR and OSR will choose their cooperative selling and qualifying efforts depending on each other's marginal productivity under the Target-Value wage. Since they are receiving incentives on both cooperative sales and behavioral terms (e.g., number of leads), they will increase their cooperative selling and qualifying efforts when the other sides' cooperative efforts' marginal productivity. It implies that the Target-Value wage fosters reps to cooperative intimately to know each others' marginal productivity.

From a managerial viewpoint, my Target-Value wage, sales plus outcome-based incentive and behavioral-based incentive and penalty, will be one of the solutions for the new ISR and OSR's cooperative sales. My proposed compensation scheme can assist managers in setting a collaborative management system for the new structure of independent ISR and OSR. In practice, the firm may adopt a high-technology CRM program to connect the two sales groups, or they may hold annual events to build up colleagueship among ISR and OSR. Along with such supportive programs, Target-Value wage will foster ISR and OSR to exchange suitably qualified leads when 
necessary for firms' higher profits and potential consumers' benefits. I obtain intriguing managerial insights on ISR and OSR's strategy and firm's outcomes based on the analytical model analysis.

Future research can extend this paper in many directions. More specifically, empirical studies of the suggested compensation plan in a lab will be worthwhile to show if the propositions are valid. During the empirical study, any possible practical precautions can be discovered before applying to an actual firm. Second, alternative functional forms of sales function may be investigated. In this paper, I assume a linear sales function, but the sales function forms may vary from industries. Third, it would be intriguing to investigate information asymmetry condition. In this paper, I assume ISR and OSR know each others' marginal productivity or risk aversion. However, if salespeople do not know each others' marginal productivity and risk aversion, the optimal strategy of working effort will be different. 


\section{References}

Ahearne M, MacKenzie SB, Podsakoff PM, Mathieu JE, Lam SK (2010a) The role of consensus in sales team performance. Journal of Marketing Research 47(3):458-469.

Ahearne M, Rapp A, Hughes DE, Jindal R (2010b) Managing sales force product perceptions and control systems in the success of new product introductions. J. Marketing Res. $47(4): 764-776$.

Albers S (1996) Optimization models for salesforce compensation. Eur. J. Oper. Res. $89(1): 1-17$.

Alvarez CM, Taylor KA, Rauseo NA (2015) Creating thoughtful salespeople: Experiential learning to improve critical thinking skills in traditional and online sales education. Marketing Education Rev. 25(3):233-243.

Anderson E, Oliver RL (1987) Perspectives on behavior-based versus outcome-based salesforce control systems. J. Marketing 51(4):76-88.

Arli D, Bauer C, Palmatier RW (2018) Relational selling: Past, present and future. Industrial Marketing Management 69:169-184.

Basu AK, Kalyanaram G (1990) On the relative performance of linear versus nonlinear compensation plans. Internat. J. Res. Marketing 7(2-3):171-178.

Basu AK, Lal R, Srinivasan V, Staelin R (1985) Salesforce compensation plans: An agency theoretic perspective. Marketing Sci. 4(4):267-291.

Bendapudi N, Leone RP (2002) Managing business-to-business customer relationships following key contact employee turnover in a vendor firm. Journal of Marketing 66(2):83101.

Caldieraro F, Coughlan AT (2009) Optimal sales force diversification and group incentive payments. Marketing Science 28(6):1009-1026.

Cespedes FV, Marsh R (2017) Find the right metrics for your sales team. Harvard Bus. Rev. Digital Article:2-5. 
Challagalla GN, Shervani TA (1996) Dimensions and types of supervisory control: effects on salesperson performance and satisfaction. J. Marketing 60(1):89-105.

Chung DJ, Huber I, Murthy V, Sunku V, Weber M (2019) Setting better sales goals with analytics. Harvard Bus. Rev. Digital Article:2-5.

Chung DJ, Steenburgh T, Sudhir K (2013) Do bonuses enhance sales productivity? a dynamic structural analysis of bonus-based compensation plans. Marketing Sci. $33(2): 165-187$.

Churchill Jr GA, Ford NM, Hartley SW, Walker Jr OC (1985) The determinants of salesperson performance: A meta-analysis. Journal of marketing research 22(2):103-118.

Coughlan AT, Narasimhan C (1992) An empirical analysis of sales-force compensation plans. J. Bus. Jan(1):93-121.

Cravens DW, Ingram TN, LaForge RW, Young CE (1993) Behavior-based and outcomebased salesforce control systems. J. Marketing 57(4):47-59.

Dai T, Jerath K (2013) Salesforce compensation with inventory considerations. Management Sci. 59(11):2490-2501.

Daljord Ø, Misra S, Nair HS (2016) Homogeneous contracts for heterogeneous agents: Aligning sales force composition and compensation. J. Marketing Res. 53(2):161-182.

Dearden JA, Lilien GL (1990) On optimal salesforce compensation in the presence of production learning effects. Internat. J. Res. Marketing 7(2-3):179-188.

Delvecchio S, Zemanek J, McIntyre R, Claxton R (2004) Updating the adaptive selling behaviours: tactics to keep and tactics to discard. J. Marketing Management 20(78):859-875.

DelVecchio SK, Zemanek JE, McIntyre RP, Claxton RP (2003) Buyers perceptions of salesperson tactical approaches. J. Personal Selling 63 Sales Management 23(1):39-49.

Dixon AL, Gassenheimer JB, Barr TF (2002) Bridging the distance between us: How initial responses to sales team conflict help shape core selling team outcomes. Journal of Personal Selling 83 Sales Management 22(4):247-257. 
Fang E, Palmatier RW, Evans KR (2008) Influence of customer participation on creating and sharing of new product value. J. Acad. Marketing Sci. 36(3):322-336.

Farley JU (1964) An optimal plan for salesmen's compensation. J. Marketing Res. 1(2):3943.

Godes D (2003) In the eye of the beholder: An analysis of the relative value of a top sales rep across firms and products. Marketing Sci. 22(2):161-187.

Gonik J (1978) Tie salesmens bonuses to their forecasts. Harvard Bus. Rev. 56(3):116-123.

Green J (2013a) Sales moves beyond face-to-face deals, onto the web. https : //www.bloomberg.com/news/articles/2013-01-10/sales-moves-beyond-faceto - face - deals - onto - the $-w e b$.

Green J (2013b) Willy loman turns to windows as web spurs sales changes. https : //www.bloomberg.com/news/articles/2013 - 01 - 02/willy - loman - turns - to windows - as - web-spurs - sales - changes .

Grewal R, Lilien GL, Bharadwaj S, Jindal P, Kayande U, Lusch RF, Mantrala M, Palmatier RW, Rindfleisch A, Scheer LK, et al. (2015) Business-to-business buying: Challenges and opportunities. Customer needs and Solutions 2(3):193-208.

Guzzo RA, Dickson MW (1996) Teams in organizations: Recent research on performance and effectiveness. Annual review of psychology 47(1):307-338.

Hauser JR, Simester DI, Wernerfelt B (1996) Internal customers and internal suppliers. J. Marketing Res. 268-280.

Hauser JR, Wernerfelt B (1990) An evaluation cost model of consideration sets. J. Consumer Res. 16(4):393-408.

Heiman A (2010) The economics of demonstrations: The effect of competition on demonstration and pricing strategies. Marketing Lett. 21(4):351-363.

Heiman A, Muller E (1996) Using demonstration to increase new product acceptance: Controlling demonstration time. J. Marketing Res. 33(4):422-430.

Hohenberg S, Homburg C (2016) Motivating sales reps for innovation selling in different cultures. J. Marketing 80(2):101-120. 
Holmes TL, Srivastava R (2002) Effects of job perceptions on job behaviors: Implications for sales performance. Industrial Marketing Management 31(5):421-428.

Holmstrom B (1979) Moral hazard and observability. Bell J. Econom. 10(1):74-91.

Holmstrom B, Milgrom P (1987) Aggregation and linearity in the provision of intertemporal incentives. Econometrica Mar(1):303-328.

Jackson DW, Tax SS (1995) Managing the industrial salesforce culture. J. Bus. ES Industrial Marketing 10(2):34-47.

Jackson Jr DW, Keith JE, Schlacter JL (1983) Evaluation of selling performance: A study of current practices. Journal of Personal Selling \& Sales Management 3(2):42-51.

Jain S (2012) Self-control and incentives: An analysis of multiperiod quota plans. Marketing Sci. 31(5):855-869.

John G, Weitz B (1989) Salesforce compensation: An empirical investigation of factors related to use of salary versus incentive compensation. J. Marketing Res. 26(1):1-14.

Johnson EJ, Payne JW (1985) Effort and accuracy in choice. Management Sci. 31(4):395414 .

Joseph K, Thevaranjan A (1998) Monitoring and incentives in sales organizations: An agency-theoretic perspective. Marketing Sci. 17(2):107-123.

Kahneman D, Frederick S, Holyoak K, Morrison R (2005) The cambridge handbook of thinking and reasoning.

Kalra A, Shi M, Srinivasan K (2003) Salesforce compensation scheme and consumer inferences. Management Sci. 49(5):655-672.

Kozlenkova IV, Palmatier RW, Fang E, Xiao B, Huang M (2017) Online relationship formation. Journal of Marketing 81(3):21-40.

Krafft M, DeCarlo TE, Poujol FJ, Tanner Jr JF (2012) Compensation and control systems: A new application of vertical dyad linkage theory. Journal of Personal Selling $\& 5$ Sales Management 32(1):107-115.

Krogue K (2013) What is inside sales? the definition of inside sales. Forbes, https : 
//www.forbes.com/sites/kenkrogue/2013/02/26/what - is - inside - salesthe definition - of - inside-sales .

Kundu SM (2019) How to create an outstanding b2b cx. https : //www.the - futureof - commerce.com/2019/03/04/how - to - create - outstanding - b2b-cx/ .

Lal R, Srinivasan V (1993) Compensation plans for single-and multi-product salesforces: An application of the holmstrom-milgrom model. Management Sci. 39(7):777-793.

Lal R, Staelin R (1986) Salesforce compensation plans in environments with asymmetric information. Marketing sci. 5(3):179-198.

Lemon KN, Verhoef PC (2016) Understanding customer experience throughout the customer journey. Journal of marketing 80(6):69-96.

Lim N, Chen H (2014) When do group incentives for salespeople work? Journal of Marketing Research 51(3):320-334.

Long MM, Tellefsen T, Lichtenthal JD (2007) Internet integration into the industrial selling process: A step-by-step approach. Industrial Marketing Management 36(5):676-689.

Mantrala M (2014) Sales force productivity models. The History Of Marketing Science, 427-462 (World Scientific).

Mantrala M, Albers S (2012) Impact of the internet on b2b sales force size and structure. Handbook of business-to-business marketing. Edward Elgar, Northhampton 539-555.

Mantrala M, Raman K (1990) Analysis of a sales force incentive plan for accurate sales forecasting and performance. Internat. J. Res. Marketing 7(2-3):189-202.

Mantrala M, Raman K, Desiraju R (1997) Sales quota plans: Mechanisms for adaptive learning. Marketing Lett. 8(4):393-405.

Mantrala M, Sinha P, Zoltners AA (1994) Structuring a multiproduct sales quota-bonus plan for a heterogeneous sales force: A practical model-based approach. Marketing Sci. 13(2):121-144.

Mishra BK, Prasad A (2004) Centralized pricing versus delegating pricing to the salesforce under information asymmetry. Marketing Sci. 23(1):21-27.

Misra S, Coughlan AT, Narasimhan C (2005) Salesforce compensation: An analytical and 
empirical examination of the agency theoretic approach. Quant. Marketing Econom. $3(1): 5-39$.

Misra S, Nair HS (2011) A structural model of sales-force compensation dynamics: Estimation and field implementation. Quant. Marketing Econom. 9(3):211-257.

Mohammed S, Dumville BC (2001) Team mental models in a team knowledge framework: Expanding theory and measurement across disciplinary boundaries. Journal of Organizational Behavior: The International Journal of Industrial, Occupational and Organizational Psychology and Behavior 22(2):89-106.

Moon MA, Armstrong GM (1994) Selling teams: A conceptual framework and research agenda. Journal of Personal Selling 8 Sales Management 14(1):17-30.

Moorman M, Bernewitz T, Solem M, Curry T (2012) Building a customer-focused growth engine: Establishing sales force effectiveness priorities. Selling Power Magazine, ZS Associates 6-12.

Moorman MB, Albrecht C (2008) Team selling: Getting incentive compensation right. Velocity 10(2):33-37.

Moxley JH, Ericsson KA, Charness N, Krampe RT (2012) The role of intuition and deliberative thinking in experts superior tactical decision-making. Cognition 124(1):72-78.

Mullins RR, Ahearne M, Lam SK, Hall ZR, Boichuk JP (2014) Know your customer: How salesperson perceptions of customer relationship quality form and influence account profitability. J. Marketing 78(6):38-58.

Murthy P, Mantrala M (2005) Allocating a promotion budget between advertising and sales contest prizes: An integrated marketing communications perspective. Marketing Lett. 16(1):19-35.

Oliver RL, Anderson E (1994) An empirical test of the consequences of behavior-and outcome-based sales control systems. J. Marketing 58(4):53-67.

Paesbrugghe B, Rangarajan D, Sharma A, Syam N, Jha S (2017) Purchasing-driven sales: Matching sales strategies to the evolution of the purchasing function. Industrial Marketing Management 62:171-184. 
Piercy NF, Cravens DW, Morgan NA (1998) Salesforce performance and behaviour-based management processes in business-to-business sales organizations. Eur. J. Marketing $32(1 / 2): 79-100$.

Piercy NF, Low GS, Cravens DW (2004) Consequences of sales managements behavior-and compensation-based control strategies in developing countries. J. Internat. Marketing $12(3): 30-57$.

Raju JS, Srinivasan V (1996) Quota-based compensation plans for multiterritory heterogeneous salesforces. Management Sci. 42(10):1454-1462.

Rao RC (1990) Compensating heterogeneous salesforces: Some explicit solutions. Marketing Sci. 9(4):319-341.

Rapp A, Agnihotri R, Forbes LP (2008) The sales force technology-performance chain: The role of adaptive selling and effort. J. Personal Selling \& Sales Management 28(4):335350.

Rapp A, Ahearne M, Mathieu J, Schillewaert N (2006) The impact of knowledge and empowerment on working smart and working hard: The moderating role of experience. Internat. J. Res. Marketing 23(3):279-293.

Rapp TL, Bachrach DG, Rapp AA, Mullins R (2014) The role of team goal monitoring in the curvilinear relationship between team efficacy and team performance. Journal of Applied Psychology 99(5):976.

Rubel O, Prasad A (2015) Dynamic incentives in sales force compensation. Marketing Sci. $35(4): 676-689$.

Rutherford BN, Marshall GW, Park J (2014) The moderating effects of gender and inside versus outside sales role in multifaceted job satisfaction. Journal of Business Research $67(9): 1850-1856$.

Salesforcecom (????) State of sales .

Saxe R, Weitz BA (1982) The soco scale: A measure of the customer orientation of salespeople. Journal of marketing research 19(3):343-351. 
Schembari J (2007) Test drive that bentley? So whats in it for me? New York Times (http://www. nytimes.com/2007/07/08/automobiles/08FREE.html) .

Schmitz C (2013) Group influences of selling teams on industrial salespeoples cross-selling behavior. Journal of the Academy of Marketing Science 41(1):55-72.

Schöttner A (2016) Optimal sales force compensation in dynamic settings: Commissions vs. bonuses. Management Sci. 63(5):1529-1544.

Schreiner L (2018) The effects of remuneration and reward systems on employee motivation in luxembourg. Technical report, EIKV-Schriftenreihe zum Wissens-und Wertemanagement.

Sheth JN, Sharma A (2008) The impact of the product to service shift in industrial markets and the evolution of the sales organization. Industrial Marketing Management $37(3): 260-269$.

Shi H, Sridhar S, Grewal R, Lilien G (2017) Sales representative departures and customer reassignment strategies in business-to-business markets. Journal of Marketing 81(2):2544.

Shugan SM (1980) The cost of thinking. J. Consumer Res. 7(2):99-111.

Simon HA, Newell A (1971) Human problem solving: The state of the theory in 1970. Amer. Psych. 26(2):145.

Sincavage D (2019) Inside sales representatives: Must-have skills. https : //www.tenfold.com/sales - performance/inside - sales - representative .

Spiro RL, Weitz BA (1990) Adaptive selling: Conceptualization, measurement, and nomological validity. J. Marketing Res. 27(1):61-69.

Srinivasan V (1981) An investigation of the equal commission rate policy for a multi-product salesforce. Management Sci. 27(7):731-756.

Steenburgh TJ, Avery J, Dahod N (2009) Hubspot: Inbound marketing and web 2.0. HBS Case (509-049).

Steward MD, Narus JA, Roehm ML, Ritz W (2019) From transactions to journeys and 
beyond: The evolution of b2b buying process modeling. Industrial Marketing Management .

Strategies G (2019) The true cost of losing a customer. https : //www.tti - global research.co.uk/true - cost-losing - customer - and - how - keep - them-good .

Sujan H, Weitz BA, Kumar N (1994) Learning orientation, working smart, and effective selling. J. Marketing 58(3):39-52.

Syam NB, Hess JD, Yang Y (2013) Sales contests versus quotas with imbalanced territories. Marketing Lett. 24(3):229-244.

Szymanski DM (1988) Determinants of selling effectiveness: the importance of declarative knowledge to the personal selling concept. J. Marketing 52(1):64-77.

Thaichon P, Surachartkumtonkun J, Quach S, Weaven S, Palmatier RW (2018) Hybrid sales structures in the age of e-commerce. Journal of Personal Selling ES Sales Management $38(3): 277-302$.

Toman N, Adamson B, Gomez C (2017) The new sales imperative. Harvard Business Review 95(2):118-125.

Weitz BA, Sujan H, Sujan M (1986) Knowledge, motivation, and adaptive behavior: A framework for improving selling effectiveness. J. Marketing 50(4):174-191.

Yang Y, Syam NB, Hess JD (2013) Thrill of victory and agony of defeat: Emotional rewards and sales force compensation. Quant. Marketing Econom. 11(4):379-402.

Zoltners A, Sinha P, Lorimer S (2013) The growing power of inside sales. https : //hbr.org/2013/07/the - growingpower - of - inside - sa .

Zoltners AA, Sinha P, Lorimer SE (2019) 7 ways sales teams can set better calls. Harvard Bus. Rev. Digital Articles:2-4.

ZSAssociates, RealityWorks (2014) Inside sales compensation practices. http : //realityworksgroup.com/wp - content/uploads/2015/03/Inside - Sales Compensation-Practices_2014-Insights-from-the-High-Tech-Industry.pdf 


\section{Appendices}

\subsection{Proposition Proofs of Essay 1}

\subsubsection{Proof of Proposition 1}

Proof. of Proposition 1 Sales Rep's Strategy under SC Plan

(a) Under SC plan, by taking the first order condition on the sales reps' Certainty Equivalent and firm's Certainty Equivalent equations, I can obtain the optimal points for selling and thinking efforts.

$$
x_{j}=\frac{w_{1 j}\left(\delta_{j}+u_{j} e_{1 j}+\phi_{j} e_{2 j}\right)}{c_{1 j} e_{1 j}^{2}+c_{2 j} e_{2 j}^{2}+c_{j}}, \quad e_{1 j}=\frac{w_{1 j} u_{j}}{c_{1 j} x_{j}}, \quad e_{2 j}=\frac{w_{1 j} \phi_{j}}{c_{2 j} x_{j}}
$$

If I perfectly solve the three equations above, then I can derive below:

$$
x_{j}^{*}=\frac{w_{1 j}^{*} \delta_{j}}{c_{j}}, \quad e_{1 j}^{*}=\frac{u_{j} c_{j}}{c_{1 j} \delta_{j}}, \quad e_{2 j}^{*}=\frac{\phi_{j} c_{j}}{c_{2 j} \delta_{j}}
$$

Since $e_{1 j}^{*}$ and $e_{1 j}^{*}$ are not functions of $w_{1 j}$, the incentive does not affect both thinking efforts. Consequently, $\beta=\delta+u_{j} e_{1 j}+\phi_{j} e_{2 j}$ is also not a function of $w_{1 j}$, and so the incentive does not affect selling effectiveness either.

(b) Total effort allocation: Based on the equation 6.1 above,

1) When $0<\frac{\delta_{j}}{c_{j}}<1$,

$$
x_{j}^{*} \downarrow \text { by } x_{j}^{*}=\frac{w_{1 j}^{*} \delta_{j}}{c_{j}}, \quad e_{1 j}^{*} \uparrow \text { by } e_{1 j}^{*}=\frac{u_{j} c_{j}}{c_{1 j} \delta_{j}}, \quad e_{2 j}^{*} \uparrow \quad \text { by } e_{2 j}^{*}=\frac{\phi_{j} c_{j}}{c_{2 j} \delta_{j}}
$$

2) When $\frac{\delta_{j}}{c_{j}}=1,6.1$ is proving per se.

3) When $1<\frac{\delta_{j}}{c_{j}}, x_{j}^{*} \uparrow, e_{1 j}^{*} \downarrow$, and $e_{2 j}^{*} \downarrow$ by equation 6.1 . 


\subsubsection{Proof of Proposition 2}

\section{Proof. of Proposition Comparison between Traditional and New Model}

In the new model with adoptable selling effectiveness, the sales function is as

$$
S_{j}^{*}=\alpha_{j}+\frac{w_{j}^{*} \delta_{j}}{c_{j}}\left(\delta_{j}+u_{j} e_{1 j}^{*}+\phi_{j} e_{2 j}^{*}\right)+\varepsilon_{j}=\alpha_{j}+w_{j}^{*}\left(\frac{\delta_{j}^{2}}{c_{j}}+\frac{u_{j}^{2}}{c_{1 j}}+\frac{\phi_{j}^{2}}{c_{2 j}}\right)+\varepsilon_{j}
$$

where $x^{*}=\frac{w_{j}^{*} \delta_{j}}{c_{j}}, w_{j}^{*}=\frac{\delta_{j} c_{1 j} c_{2 j}\left(\delta_{j}-\nu\right)+c_{j}\left(c_{1 j} \phi_{j}^{2}+c_{2 j} u_{j}^{2}\right)}{2\left(c_{j} c_{1 j} c_{2 j} \mu_{j} \sigma_{j}^{2}+c_{1 j} c_{2 j} \delta_{j}^{2}+c_{j}\left(c_{1 j} \phi_{j}^{2}+c_{2 j} u_{j}^{2}\right)\right)}, \beta_{j}^{*}=\delta_{j}+u_{j} e_{1 j}^{*}+\phi_{j} e_{2 j}^{*}$, $e_{1 j}^{*}=\frac{u_{j} c_{j}}{c_{1 j} \delta_{j}}$, and $e_{2 j}^{*}=\frac{\phi_{j} c_{j}}{c_{2 j} \delta_{j}}$. With taking partial derivative for the new model's selling effectiveness, I can obtain;

$$
\frac{\partial \beta_{j}^{*}}{\partial \delta_{j}}=1-\frac{c_{j}}{\delta_{j}^{2}}\left(\frac{u_{j}^{2}}{c_{1 j}}+\frac{\phi_{j}^{2}}{c_{2 j}}\right) \Rightarrow \begin{cases}\frac{\partial \beta_{j}^{*}}{\partial \delta_{j}}<0, & \text { if } \delta_{j}<\sqrt{c_{j}\left(\frac{u_{j}^{2}}{c_{1 j}}+\frac{\phi_{j}^{2}}{c_{2 j}}\right)} \\ \frac{\partial \beta_{j}^{*}}{\partial \delta_{j}} \geq 0, & \text { if } \delta_{j} \geq \sqrt{c_{j}\left(\frac{u_{j}^{2}}{c_{1 j}}+\frac{\phi_{j}^{2}}{c_{2 j}}\right)}\end{cases}
$$

On the contrary, the traditional models' optimal selling effectiveness will be always increase when the rep j's base ability increases $\left(\because \frac{\partial \beta_{t r d, j}^{*}}{\partial \delta_{j}}=1>0\right.$ where $\left.\beta_{j}=\delta_{j}\right)$.

\subsubsection{Proof of Proposition 3}

\section{Proof. of Proposition Comparison between Traditional and New Model}

With taking partial derivative for the new model's optimal incentive, I can obtain; $\frac{\partial w_{j}^{*}}{\partial \delta_{j}}=\frac{v c_{1 j} c_{2 j} \delta_{j}^{2}+2 c_{j} c_{2 j} c_{2 j} \mu_{j} \sigma_{j}^{2} \delta_{j}-v c_{j}\left(\mu_{j} \sigma_{j}^{2}+\left(c_{2 j} u_{j}^{2}+c_{1 j} \phi_{j}^{2}\right)\right)}{2\left(c_{1 j} c_{2 j} \delta_{j}^{2}+c_{j}\left(\mu_{j} \sigma_{j}^{2}+\left(c_{2 j} u_{j}^{2}+c_{1 j} \phi_{j}^{2}\right)\right)\right)^{2}}$. There are two points of $\delta_{j}$. Since $\delta_{j}>0$, a negative value is not valid. Let's define $\tilde{\delta}$ be the positive value $\tilde{\delta}=-\frac{c_{j} \mu_{j} \sigma_{j}^{2}}{v}+\frac{\sqrt{c_{j} c_{1 j} c_{2 j}\left(c_{j} c_{1 j} c_{2 j} \mu_{j} \sigma_{s j}^{2}+v^{2}\left(c_{1 j} \phi_{j}^{2}+c_{2 j} u_{j}^{2}+\mu_{j} \sigma_{j}^{2}\right)\right)}}{c_{1 j} c_{2 j} v}$. Then, $\therefore \begin{cases}\frac{\partial w_{j}^{*}}{\partial \delta_{j}}<0, & \text { if } \delta_{j}<\tilde{\delta} \\ \frac{\partial w_{j}^{*}}{\partial \delta_{j}} \geq 0, & \text { if } \delta_{j} \geq \tilde{\delta}\end{cases}$

On the contrary, the traditional models' optimal incentive will be always increase when the rep j's base ability increases $\left(\because \frac{\partial w_{t r d, j}^{*}}{\partial \delta_{j}}=\frac{c_{j} \mu_{j} \sigma_{j}^{2}}{\left(\delta_{j}^{2}+c_{j} \mu_{j} \sigma_{j}^{2}\right)^{2}}>0\right)$. 


\subsubsection{Proof of Lemma 4.1}

\section{Proof. of Lemma 4.1 Two Reps' Selling Effectiveness}

Let $\beta_{1}^{*}$ is Rep 1's selling effectiveness and $\beta_{2}^{*}$ is the rep 2's. Then, $\beta_{1}^{*}=2 \delta+\frac{c}{2 \delta}\left(\frac{c_{1} \phi^{2}+c_{2} u^{2}}{c_{1} c_{2}}\right)$ and $\beta_{2}^{*}=\delta+\frac{c}{\delta}\left(\frac{c_{1} \phi^{2}+c_{2} u^{2}}{c_{1} c_{2}}\right)$.

If $\beta_{1}^{*}>\beta_{2}^{*}$, equation as below should be true.

$$
2 \delta+\frac{c}{2 \delta}\left(\frac{c_{1} \phi^{2}+c_{2} u^{2}}{c_{1} c_{2}}\right)>\delta+\frac{c}{\delta}\left(\frac{c_{1} \phi^{2}+c_{2} u^{2}}{c_{1} c_{2}}\right)
$$

I can obtain $\delta>\sqrt{\frac{c\left(c_{1} \phi^{2}+c_{2} u^{2}\right)}{2 c_{1} c_{2}}}(\because \delta>0)$ by solving equation 6.2. Therefore,

$$
\left\{\begin{array}{ll}
\beta_{1} \geq \beta_{2}, & \text { if } \delta \geq \sqrt{\frac{c_{\left(c_{1} \phi^{2}+c_{2 j} u^{2}\right)}^{2 c_{1} c_{2}}}{2 c_{1} c_{2}}} \\
\beta_{1}<\beta_{2}, & \text { if } \delta<\sqrt{\frac{c\left(c_{1} \phi^{2}+c_{2} u^{2}\right)}{2}}
\end{array} .\right.
$$

\subsubsection{Proof of Proposition 4}

\section{Proof. of Proposition Sales Rep's Strategy under MD-C Plan}

The salespeople's Certainty Equivalent under the multidimensional plan is below:

$$
\begin{array}{r}
C E_{m j}=w_{0}+w_{1 j} S\left(e_{1 j}, e_{2 j}, x_{j}\right)+w_{2 j} K\left(e_{1 j}, e_{2 j}, x_{j}\right) \\
-\left(\frac{c_{1 j}\left(e_{1 j} x_{j}\right)^{2}}{2}+\frac{c_{2 j}\left(e_{2 j} x_{j}\right)^{2}}{2}+\frac{c_{j} x_{j}^{2}}{2}\right)-\frac{\mu_{j}}{2} w_{1 j}^{2} \sigma_{j}^{2}
\end{array}
$$

By taking the first order condition from the CE above, I can derive following as

$$
x_{j}^{*}=\frac{w_{1 j}^{*} \delta_{j}}{c_{j}}, \quad e_{1 j}^{*}=\frac{u_{j} c_{j}}{c_{1 j} \delta_{j}}+\frac{\gamma_{j} c_{j}}{c_{1 j} \delta_{j}} \frac{w_{2 j}^{*}}{w_{1 j}^{*}}, \quad e_{2 j}^{*}=\frac{\phi_{j} c_{j}}{c_{2 j} \delta_{j}}+\frac{\gamma_{j} c_{j}}{c_{2 j} \delta_{j}} \frac{w_{2 j}^{*}}{w_{1 j}^{*}}
$$

(a) Since $\frac{\partial x_{j}^{*}}{\partial w_{1 j}}>0, \frac{\partial e_{1 j}^{*}}{\partial w_{1 j}}<0, \frac{\partial e_{2 j}^{*}}{\partial w_{1 j}}<0$, the rep will increase selling effort but decrease thinking efforts by increasing the incentive on sales $(\mathrm{S})$.

(b) Since $\frac{\partial x_{j}^{*}}{\partial w_{2 j}}=0, \frac{\partial e_{1 j}^{*}}{\partial w_{2 j}}>0, \frac{\partial e_{2 j}^{*}}{\partial w_{2 j}}>0$, the rep will not change selling effort but increase thinking efforts by increasing the incentive on activity $(\mathrm{K})$. 
(c) $e_{1 j, s c}+{ }_{2 j, s c}<e_{1 j, m c}+{ }_{2 j, m c}$

$\mathrm{c}-1) e_{1 j, s c}^{*}<e_{1 j, m c}^{*}, \because \frac{u_{j} c_{j}}{c_{1 j} \delta_{j}}<\frac{c_{j} u_{j}}{c_{1 j} \delta_{j}}+\frac{c_{j} \gamma_{1 j}}{c_{1 j} \delta_{j}} \frac{w_{2 j, m c}^{*}}{w_{1 j, m c}^{*}}$

$\mathrm{c}-2) e_{2 j, s c}^{*}<e_{2 j, m c}^{*}, \because \frac{\phi_{j} c_{j}}{c_{2 j} \delta_{j}}<\frac{c_{j} u_{j}}{c_{2 j} \delta_{j}}+\frac{c_{j} \gamma_{2 j}}{c_{2 j} \delta_{j}} \frac{w_{2 j, m c}^{*}}{w_{1 j, m c}^{*}}$

\subsubsection{Proof of Proposition 5}

\section{Proof. of Proposition 5 Comparison between SC and MD-C Plans}

(a) Optimal Sales Incentives $\left(w_{s c, 1}^{*}, w_{m c, 1}^{*}\right)$

The optimal incentives for each plan are as below;

Under SC plan: $w_{s c, 1}^{*}=\frac{p-\delta_{j} \nu_{j} c_{1 j} c_{2 j}}{2\left(p+c_{j} c_{1 j} c_{2 j} \mu_{j} \sigma_{s j}^{2}\right)}$ where $p_{j}=c_{1 j} c_{2 j} \delta_{j}^{2}+c_{j}\left(c_{1 j} \phi_{j}^{2}+c_{2 j} u_{j}^{2}\right)$.

Under MD-C plan: $w_{m c, 1}^{*}=\frac{y-\delta_{j} \nu_{j} b}{2\left(y+b c_{j} \mu_{j} \sigma_{s j}^{2}\right)}$ where $\hat{b}_{j}=\gamma_{1 j}^{2} c_{2 j}+\gamma_{2 j}^{2} c_{1 j}+c_{1 j} c_{2 j} \mu_{j} \sigma_{k j}^{2}$, and $y_{j}=c_{j}\left(\gamma_{1 j} \phi_{j}-\gamma_{2 j} u\right)^{2}+c_{j}\left(c_{1 j} \phi_{j}^{2}+c_{2 j} u_{j}^{2}\right) \mu_{j} \sigma_{k j}^{2}+\delta_{j}^{2}\left(\gamma_{1 j}^{2} c_{2 j}+\gamma_{2 j}^{2} c_{1 j}+c_{1 j} c_{2 j} \mu_{j} \sigma_{k j}^{2}\right)$.

To prove $w_{s c, 1}^{*}>w_{m c, 1}^{*}$, I need to show if equation 6.5 is true or not.

$$
\frac{p_{j}-\delta_{j} \nu_{j} c_{1 j} c_{2 j}}{2\left(p_{j}+c_{j} c_{1 j} c_{2 j} \mu_{j} \sigma_{s j}^{2}\right)}>\frac{y_{j}-\delta_{j} \nu_{j} \hat{b}_{j}}{2\left(y_{j}+\hat{b}_{j} c_{j} \mu_{j} \sigma_{s j}^{2}\right)}
$$

The equation 6.5 can be shown as 6.6 by transposing and simplifying.

$$
c_{1 j}^{2} \gamma_{2 j}^{2} \phi_{j}^{2}+c_{2 j}^{2} \gamma_{1 j}^{2} u_{j}^{2}+2 c_{1 j} c_{2 j} \gamma_{1 j} \gamma_{2 j} u_{j} \phi_{j}>0
$$

Since the equation 6.6 is larger than $0\left(\because\left(c_{1 j} \gamma_{2 j} \phi_{j}+c_{2 j} \gamma_{1 j} u_{j}\right)^{2}>0\right)$, equation 6.5 is true. Therefore, $w_{s c, 1}^{*}>w_{m c, 1}^{*}$ for $\forall \delta_{j}$.

(b) Selling Effort $\left(x_{s c, j}^{*}, x_{m c, j}^{*}\right)$

The optimal selling efforts for each plan are as below;

Under SC plan: $x_{s c, j}^{*}=\frac{\delta_{j}}{c_{j}} \frac{p_{j}-\delta_{j} \nu_{j} c_{1 j} c_{2 j}}{2\left(p_{j}+c_{j} c_{1 j} c_{2 j} \mu_{j} \sigma_{s j}^{2}\right)}$

Under MD-C plan: $x_{m c, j}^{*}=\frac{\delta_{j}}{c_{j}} \frac{y_{j}-\delta_{j} \nu_{j} b_{j}}{2\left(y_{j}+b_{j} c_{j} \mu_{j} \sigma_{s j}^{2}\right)}$.

If $x_{s c, 1}^{*}>x_{m c, 1}^{*}, \frac{p_{j}-\delta_{j} \nu_{j} c_{1 j} c_{2 j}}{2\left(p_{j}+c_{j} c_{1 j} c_{2 j} \mu_{j} \sigma_{s j}^{2}\right)}>\frac{y_{j}-\delta_{j} \nu_{j} b_{j}}{2\left(y_{j}+b_{j} c_{j} \mu_{j} \sigma_{s j}^{2}\right)}$ which is equal to the equation 6.5 should be true. Since I show that equation 6.5 is true above, I can also confirm that 
$x_{s c, 1}^{*}>x_{m c, 1}^{*}$ for $\forall \delta_{j}$.

(c) Selling Effectiveness $\left(\beta_{s c, j}^{*}, \beta_{m c, j}^{*}\right)$

The optimal selling effectiveness for each plan are as below;

Under SC plan: $\beta_{s c, j}^{*}=\delta_{j}+\frac{\delta_{j}}{c_{j}}\left(\frac{u_{j}}{c_{1 j}}+\frac{\phi_{j}}{c_{2 j}}\right)$

Under MD-C plan: $\beta_{m c, j}^{*}=\delta_{j}+\frac{\delta_{j}}{c_{j}}\left(\frac{u_{j}}{c_{1 j}}+\frac{\phi_{j}}{c_{2 j}}\right)+\frac{\delta_{j}}{c_{j}}\left(\frac{\gamma_{1 j}}{c_{1 j}}+\frac{\gamma_{2 j}}{c_{2 j}}\right) \frac{w_{m c, 2 j}^{*}}{w_{m c, 1 j}^{*}}$ where $\frac{w_{2 j}^{*}}{w_{1 j}^{*}}=\frac{k_{j}}{b_{j}}\left(\frac{1}{2}-w_{m c, 1 j}^{*}\right) \frac{1}{w_{m c, 1 j}^{*}}=\frac{k_{j}}{b_{j}}\left(\frac{1}{2 w_{m c, 1 j}^{*}}-1\right)$.

To prove $\beta_{s c, j}^{*}>\beta_{m c, j}^{*}$, I need to show if the following equation is true or not:

$$
\delta_{j}+\frac{\delta_{j}}{c_{j}}\left(\frac{u_{j}}{c_{1 j}}+\frac{\phi_{j}}{c_{2 j}}\right)>\delta_{j}+\frac{\delta_{j}}{c_{j}}\left(\frac{u_{j}}{c_{1 j}}+\frac{\phi_{j}}{c_{2 j}}\right)+\frac{\delta_{j}}{c_{j}}\left(\frac{\gamma_{1 j}}{c_{1 j}}+\frac{\gamma_{2 j}}{c_{2 j}}\right) \frac{w_{m c, 2 j}^{*}}{w_{m c, 1 j}^{*}}
$$

Equation 6.7 is equal to the equation 6.8 below.

$$
b_{j}\left(\delta_{j} \nu_{j}+\mu_{j} c_{j} \sigma_{s j}^{2}\right)<0
$$

Equation 6.8 is not true because $b_{j}>0, \delta_{j} \nu_{j}>0$, and $\mu_{j} c_{j} \sigma_{s j}^{2}>0$. Therefore, $\beta_{s c, 1}^{*}<\beta_{m c, 1}^{*}$ for $\forall \delta_{j}$.

(d) Sales outcome $s_{s c, j}^{*}, s_{m c, j}^{*}$

The optimal sales for each plan are as below;

Under SC plan: $s_{s c, j}^{*}=\alpha_{j}+\left(\delta_{j}+\frac{c_{j}}{\delta_{j}} o_{j}\right) \frac{\delta_{j}}{c_{j}} w_{s c, 1}^{*}$

Under MD-C plan: $s_{m c, j}^{*}=\alpha_{j}+\left(\delta_{j}+\frac{c_{j}}{\delta_{j}} o_{j}+\frac{c_{j}}{\delta_{j}} t_{j} \frac{w_{m c, 2 j}^{*}}{w_{m c, 1 j}^{*}}\right) \frac{\delta_{j}}{c_{j}} w_{m c, 1}^{*}=\alpha_{j}+\left(\delta_{j}+\frac{c_{j}}{\delta_{j}} o_{j}\right) \frac{\delta_{j}}{c_{j}} w_{m c, 1}^{*}+$ $\frac{c_{j}}{\delta_{j}} \frac{\delta_{j}}{c_{j}} t_{j} w_{m c, 2 j}^{*}$

where $o_{j}=\frac{u_{j}}{c_{1 j}}+\frac{\phi_{j}}{c_{2 j}}$ and $t_{j}=\frac{\gamma_{1 j}}{c_{1 j}}+\frac{\gamma_{2 j}}{c_{2 j}}$.

If $s_{s c, j}^{*}>s_{m c, j}^{*}$, the equation 6.9 should be true.

$$
\alpha_{j}+\left(\delta_{j}+\frac{c_{j}}{\delta_{j}} o_{j}\right) \frac{\delta_{j}}{c_{j}} w_{s c, 1}^{*}>\alpha_{j}+\left(\delta_{j}+\frac{\delta_{j}}{c_{j}} o_{j}\right) \frac{\delta_{j}}{c_{j}} w_{m c, 1}^{*}+t_{j} w_{m c, 2 j}^{*}
$$


By solving equation 6.9, I can obtain $\delta_{j}$ where $\delta_{j}>0$ :

$$
\delta_{j}>\sqrt{\frac{c_{j}\left(\gamma_{1 j} \phi_{j}^{2}+\gamma_{2 j} u_{j}^{2}+\left(\gamma_{1 j} c_{2 j}+\gamma_{2 j} c_{1 j}\right) \mu_{j} \sigma_{s j}^{2}-u_{j} \phi_{j}\left(\gamma_{1 j}+\gamma_{2 j}\right)\right)}{\left(c_{1 j} \gamma_{2 j} \phi_{j}+c_{2 j} \gamma_{1 j} u_{j}-c_{j}\left(\gamma_{1 j} c_{2 j}+\gamma_{2 j} c_{1 j}\right)\right)}}
$$

Define the right hand in the equation 6.10 as $\bar{\delta}$. Then, $s_{s c, 1}^{*}>s_{m c, 1}^{*}$ when $\delta_{j}>\bar{\delta}$. However, $s_{s c, 1}^{*} \leq s_{m c, 1}^{*}$ when $\delta_{j} \leq \bar{\delta}$.

\subsubsection{Proof of Proposition 6}

\section{Proof. of Proposition 6 Comparison between SC and SU plans}

Assume there are two reps $j=1,2$ where $\delta_{1}>\delta_{2} \geq \frac{1}{2}$ and all else are equal. Then, define $\delta_{1}=2 \delta_{2}$ to simplify math. The optimal commission rates on sales under SC plan for rep $1\left(w_{11}^{*}\right)$ and rep $2\left(w_{12}^{*}\right)$, and the optimal commission rate under SU plan $\left(\bar{w}_{1}^{*}\right)$ are respectively;

$$
\begin{array}{r}
w_{11}^{*}=\frac{4 \delta^{2} c_{1 j} c_{2}-2 \delta \nu c_{1} c_{2}+a c}{2\left(4 \delta^{2} c_{1} c_{2}+c b\right)}, w_{12}^{*}=\frac{\delta^{2} c_{1 j} c_{2}-\delta \nu c_{1} c_{2}+a c}{2\left(\delta^{2} c_{1} c_{2}+c b\right)}, \\
\bar{w}_{1}^{*}=\frac{5 \delta^{2} c_{1 j} c_{2}-3 \delta \nu c_{1} c_{2}+3 a c}{2\left(5 \delta^{2} c_{1} c_{2}+2 c b\right)}
\end{array}
$$

where $b=c_{1} \phi^{2}+c_{2} u+c_{1} c_{2} \mu \sigma_{s}^{2}, a=c_{1} \phi^{2}+c_{2} u$ in this proposition.

(a) Optimal Thinking Efforts

Let $\sum e_{s c}^{*}, \sum e_{s u}^{*}$ are the two reps' total optimal thinking efforts under SC plan and $\mathrm{SU}$ plan respectively.

$$
\sum e_{s c}^{*}=e_{11}^{*}+e_{12}^{*}+e_{21}^{*}+e_{22}^{*}, \quad \sum e_{s u}^{*}=\bar{e}_{11}^{*}+\bar{e}_{12}^{*}+\bar{e}_{21}^{*}+\bar{e}_{22}^{*}
$$

where $e_{11}^{*}$ is rep 1's thinking effort in qualifying and $e_{12}^{*}$ is rep 1's thinking effort in selling stage. Also, $e_{21}^{*}$ and $e_{22}^{*}$ are rep 2's thinking efforts in qualifying and sell- 
ing stage respectively. Since rep 1 and 2's optimal thinking effort choice are equal $\left(e_{11}^{*}=\bar{e}_{11}^{*}=\frac{u c}{2 c_{1} \delta}, e_{12}^{*}=\bar{e}_{12}^{*}=\frac{\phi c}{2 c_{2} \delta}, e_{21}^{*}=\bar{e}_{21}^{*}=\frac{u c}{c_{1} \delta}, e_{22}^{*}=\bar{e}_{22}^{*}=\frac{\phi c}{c_{2} \delta}\right)$, the total optimal thinking efforts under the two compensation plans are also equal $\left(\sum e_{s c}^{*}=\sum e_{s u}^{*}\right)$.

(b) Optimal Commissions

b-1) If $w_{11}^{*}<\bar{w}_{1}^{*}, \frac{4 \delta^{2} c_{1 j} c_{2}-2 \delta \nu c_{1} c_{2}+a c}{2\left(4 \delta^{2} c_{1} c_{2}+c b\right)}-\frac{5 \delta^{2} c_{1 j} c_{2}-3 \delta \nu c_{1} c_{2}+3 a c}{2\left(5 \delta^{2} c_{1} c_{2}+2 c b\right)}<0$ should be true. Once I solve the left hand side in this equation, I can obtain the equation below:

$$
2 c_{1} c_{2} \nu \delta^{2}+3 c c_{1} c_{2} \mu \sigma_{s}^{2} \delta-c b \nu
$$

By solving equation6.11 regarding $\delta$, I can obtain $\delta=\frac{-3 c \mu \sigma_{s}^{2}}{4 v}+\sqrt{\frac{c b}{2 c_{1} c_{2}}+\left(\frac{3 c \mu \sigma_{s}^{2}}{4 v}\right)^{2}}(\because$ $\delta>0)$. Then, let the right hand in the solution of $\delta$ as $\hat{\delta}$. Then, I can find the range of $\delta:\left\{\begin{array}{ll}\bar{w}_{1}^{*} \leq w_{11}^{*}, & \text { if } \delta_{2} \geq \hat{\delta} \\ w_{11}^{*}>\bar{w}_{1}^{*}, & \text { if } \delta_{2}<\hat{\delta}\end{array}\right.$.

b-2) Similarly, if $\bar{w}_{1}^{*}<w_{12}^{*}, \frac{5 \delta^{2} c_{1 j} c_{2}-3 \delta \nu c_{1} c_{2}+3 a c}{2\left(5 \delta^{2} c_{1} c_{2}+2 c b\right)}-\frac{\delta^{2} c_{1 j} c_{2}-\delta \nu c_{1} c_{2}+a c}{2\left(\delta^{2} c_{1} c_{2}+c b\right)}<0$ should be true. By solving it, I can obtain the equal equation to 6.11 .

Therefore, $\left\{\begin{array}{l}w_{12}^{*} \leq \bar{w}_{1}^{*} \leq w_{11}^{*}, \quad \text { if } \delta_{2} \geq \hat{\delta} \\ w_{11}^{*}>\bar{w}_{1}^{*}>w_{12}^{*}, \quad \text { if } \delta_{2}<\hat{\delta}\end{array}\right.$.

\section{(c) Optimal Selling Efforts}

Let $\sum x_{s c}^{*}, \sum x_{s u}^{*}$ are the two reps' total selling efforts under SC plan and SU plan respectively. Under SC plan: $\sum x_{s c}^{*}=x_{1}^{*}+x_{2}^{*}$

Under SU plan: $\sum \bar{x}_{s u}^{*}=\bar{x}_{1}^{*}+\bar{x}_{2}^{*}$

where $x_{1}^{*}, \bar{x}_{1}^{*}$ are the rep 1's selling efforts under SC plan and SU plan respectively, and $x_{2}^{*}, \bar{x}_{2}^{*}$ are rep 2's selling efforts under SC plan and SU plan respectively. To compare the total optimal selling efforts under each plan $\left(\sum x_{s c}^{*} \gtrless \sum \bar{x}_{s u}^{*}\right)$, I should know if 1) $\left.x_{1}^{*}<\bar{x}_{1}^{*}, 2\right) x_{2}^{*}<\bar{x}_{2}^{*}$, and 3) $\left|x_{1}^{*}-\bar{x}_{1}^{*}\right|>\left|x_{2}^{*}-\bar{x}_{2}^{*}\right|$ are true or not. 
c-1) If $x_{1}^{*}<\bar{x}_{1}^{*}$, the equation 6.12 should be true:

$$
\frac{4 \delta^{2} c_{1} c_{2}-2 \delta v c_{1} c_{2}+a c}{2\left(4 \delta^{2} c_{1} c_{2}+c b\right)}-\frac{5 \delta^{2} c_{1} c_{2}-3 \delta v c_{1} c_{2}+2 a c}{2\left(5 \delta^{2} c_{1} c_{2}+2 c b\right)}<0
$$

where $x_{1}^{*}=w_{11}^{*} \frac{2 \delta}{c}=\frac{4 \delta^{2} c_{1} c_{2}-2 \delta v c_{1} c_{2}+a c}{2\left(4 \delta^{2} c_{1} c_{2}+c b\right)}$, and $\bar{x}_{1}^{*}=\bar{w}_{1}^{*} \frac{2 \delta}{c}=\frac{5 \delta^{2} c_{1} c_{2}-3 \delta v c_{1} c_{2}+2 a c}{2\left(5 \delta^{2} c_{1} c_{2}+2 c b\right)}$ (note: $\left.b=c_{1} \phi^{2}+c_{2} u+c_{1} c_{2} \mu \sigma_{s}^{2}, a=c_{1} \phi^{2}+c_{2} u^{2}\right)$. Equation 6.12 can be written as 6.11 and it leads to the similar condition as above. Therefore, $\left\{\begin{array}{ll}x_{1}^{*} \geq \bar{x}_{1}^{*}, & \text { if } \delta_{2} \geq \hat{\delta}, \\ x_{1}^{*}<\bar{x}_{1}^{*}, & \text { if } \delta_{2}<\hat{\delta}\end{array}\right.$.

c-2) If $x_{2}^{*}<\bar{x}_{2}^{*}$, the equation 6.13 should be true:

$$
\frac{\delta^{2} c_{1} c_{2}-\delta v c_{1} c_{2}+a c}{2\left(\delta^{2} c_{1} c_{2}+c b\right)}-\frac{5 \delta^{2} c_{1} c_{2}-3 \delta v c_{1} c_{2}+2 a c}{2\left(5 \delta^{2} c_{1} c_{2}+2 c b\right)}<0
$$

where $x_{2}^{*}=w_{12}^{*} \frac{\delta}{c}=\frac{\delta^{2} c_{1} c_{2}-\delta v c_{1} c_{2}+a c}{2\left(\delta^{2} c_{1} c_{2}+c b\right)}$, and $\bar{x}_{2}^{*}=\bar{w}_{1}^{*} \frac{\delta}{c}=\frac{5 \delta^{2} c_{1} c_{2}-3 \delta v c_{1} c_{2}+2 a c}{2\left(5 \delta^{2} c_{1} c_{2}+2 c b\right)}$. Since equation 6.13 is equal to equation 6.11 by solving, I can conclude such that $\left\{\begin{array}{ll}x_{2}^{*} \leq \bar{x}_{2}^{*}, & \text { if } \delta \geq \hat{\delta}, \\ x_{2}^{*}>\bar{x}_{2}^{*}, & \text { if } \delta<\hat{\delta}\end{array}\right.$.

c-3) If $\left|x_{1}^{*}-\bar{x}_{1}^{*}\right|>\left|x_{2}^{*}-\bar{x}_{2}^{*}\right|$ is true, $-2 \delta^{2} c_{1} c_{2}+c b>0$ is also true $\left(\because\left(\left|x_{1}^{*}-\bar{x}_{1}^{*}\right|-\right.\right.$ $\left.\left.\left|x_{2}^{*}-\bar{x}_{2}^{*}\right|\right)=\left(-2 \delta^{2} c_{1} c_{2}+c b\right)\right)$. Since $\delta>0$, I can obtain the range of $\delta<\sqrt{\frac{c b}{2 c_{1} c_{2}}}$ for $\left|x_{1}^{*}-\bar{x}_{1}^{*}\right|>\left|x_{2}^{*}-\bar{x}_{2}^{*}\right|$ to be true.

Note that $\sqrt{\frac{c b}{2 c_{1} c_{2}}}<\hat{\delta}$. Hence, If $\hat{\delta} \leq \sqrt{\frac{c b}{2 c_{1} c_{2}}} \leq \delta, \sum x_{s c}^{*}-\sum x_{s u}^{*}=x_{1}^{*}-\bar{x}_{1}^{*}+x_{2}^{*}-$ $\bar{x}_{2}^{*} \leq 0$.

If $\delta \leq \hat{\delta} \leq \sqrt{\frac{c b}{2 c_{1} c_{2}}}, \sum x_{s c}^{*}-\sum x_{s u}^{*}=x_{1}^{*}-\bar{x}_{1}^{*}+x_{2}^{*}-\bar{x}_{2}^{*} \leq 0$.

If $\hat{\delta} \leq \delta \leq \sqrt{\frac{c b}{2 c_{1} c_{2}}}, \sum x_{s c}^{*}-\sum x_{s u}^{*}=x_{1}^{*}-\bar{x}_{1}^{*}+x_{2}^{*}-\bar{x}_{2}^{*}>0$.

Therefore, $\left\{\begin{array}{ll}\sum x_{s c}^{*} \leq \sum x_{s u}^{*}, & \text { if } \hat{\delta} \leq \sqrt{\frac{c b}{2 c_{1} c_{2}}} \leq \delta \text { or } \delta \leq \hat{\delta} \leq \sqrt{\frac{c b}{2 c_{1} c_{2}}} \\ \sum x_{s c}^{*}>\sum x_{s u}^{*}, & \text { if } \hat{\delta} \leq \delta \leq \sqrt{\frac{c b}{2 c_{1} c_{2}}}\end{array}\right.$. 
(d) Optimal Sales

Let $\breve{\beta}^{*}$ be the optimal selling effectiveness based on the optimal thinking effort choices under SU plan, and $\beta^{*}$ be the optimal selling effectiveness under SC plan. Then, the firms' overall sales from both reps under SC and SU plans are;

Under SC plan: $\sum S_{s c}^{*}=2 \alpha+\frac{2 \delta}{c} w_{11}^{*} \beta_{1}^{*}+\frac{\delta}{c} w_{12}^{*} \beta_{2}^{*}$

Under SU plan: $\sum S_{s u}^{*}=2 \alpha+\frac{2 \delta}{c} \bar{w}_{1}^{*} \breve{\beta}_{1}^{*}+\frac{\delta}{c} \bar{w}_{1}^{*} \breve{\beta}_{2}^{*}$

Then, show that $\sum S_{s c}^{*}<\sum S_{s u}^{*}$ is true under what condition. In Standard plans, $\breve{\beta^{*}}=\beta^{*}$ because the commission rate $w_{1}^{*}$ does not affect reps thinking efforts. Hence, $\sum S_{s c}^{*}-\sum S_{s u}^{*}$ can be rewritten as $2 \beta_{1}^{*}\left(w_{11}^{*}-\bar{w}_{1}^{*}\right)+\beta_{2}^{*}\left(w_{12}^{*}-\bar{w}_{1}^{*}\right)$. As I have shown in (b), $w_{11}^{*}-\bar{w}_{1}^{*}$ and $w_{12}^{*}-\bar{w}_{1}^{*}$ depend on the range of $\delta$.

d-1) If $\delta \geq \hat{\delta}$,

$\sum S_{s c}^{*}-\sum S_{s u}^{*}=2 \beta_{1}^{*}\left(w_{11}^{*}-\bar{w}_{1}^{*}\right)+\beta_{2}^{*}\left(w_{12}^{*}-\bar{w}_{1}^{*}\right)=c_{1} c_{2} \mu \sigma_{s}^{2}<0$. It is false because all parameters $\left(c_{1} c_{2} \mu \sigma_{s}\right)$ are positive. Hence, $S_{s c}^{*}>S_{s u}^{*}$ in this condition.

d-2) If $\delta<\hat{\delta}$,

$\sum S_{s c}^{*}-\sum S_{s u}^{*}=-2 \beta_{1}^{*}\left(\delta^{2} c_{1} c_{2}+c b\right)+\beta_{2}^{*}\left(4 \delta^{2} c_{1} c_{2}+c b\right)=-c_{1} c_{2} \mu \sigma_{s}^{2}<0$ which is true.

Therefore, by d-1) and d-2), $\left\{\begin{array}{ll}S_{s c}^{*} \geq S_{s u}^{*}, & \text { if } \delta \geq \hat{\delta}, \\ S_{s c}^{*}<S_{s u}^{*}, & \text { if } \delta<\hat{\delta}\end{array}\right.$.

\subsubsection{Proof of Proposition 7}

\section{Proof. of Proposition Comparison of MD-C and MD-U Plans}

Assume there are two reps $j=1,2$ where $\delta_{1}>\delta_{2} \geq \frac{1}{2}$ and all else are equal. Then, define $\delta_{1}=2 \delta_{2}$ to simplify math. Under MD-C plan, the optimal sales incentive and activity incentive are as follows respectively;

$\left\{\begin{array}{l}\text { High base ability rep; } w_{11}^{*}=\frac{4 \delta^{2} \hat{b}-2 \delta v \hat{b}+\hat{a} c}{2\left(4 \delta^{2} b+c \hat{b} \mu \sigma_{s}^{2}+\hat{a} c\right)}, w_{12}^{*}=\frac{\left(2 \delta^{2} v+c \mu \sigma_{s}^{2}\right) k}{2\left(4 \delta^{2} \hat{b}+c \hat{b} \mu \sigma_{s}^{2}+\hat{a} c\right)}, \\ \text { Low base ability rep; } w_{21}^{*}=\frac{\delta^{2} \hat{b}-\delta v \hat{b}+\hat{a} c}{2\left(\delta^{2} \hat{b}+c \hat{b} \mu \sigma_{s}^{2}+\hat{a} c\right)}, w_{22}^{*}=\frac{\left(\delta^{2} v+c \mu \sigma_{s}^{2}\right) k}{2\left(\delta^{2} \hat{b}+c \hat{b} \mu \sigma_{s}^{2}+\hat{a} c\right)}\end{array}\right.$

Where $\hat{a}=\left(\phi \gamma_{1}-u \gamma_{2}\right)^{2}+\left(c_{1} \phi^{2}+c_{2} u^{2}\right) \mu \sigma_{k}^{2}, \hat{b}=c_{2} \gamma_{1}^{2}+c_{1} \gamma_{2}^{2}+c_{1} c_{2} \mu \sigma_{k}^{2}$, and $k=$ 
$\gamma_{1} c_{2} u+\gamma_{2} c_{1} \phi$

Under MD-U plan, sales incentive and activity incentive for both reps are as follows respectively;

$\bar{w}_{1}^{*}=\frac{5 \delta^{2} \hat{b}-3 \delta v \hat{b}+2 \hat{a} c}{2\left(5 \delta^{2} \hat{b}+2 c \hat{b} \mu \sigma_{s}^{2}+2 \hat{a} c\right)}, \quad \bar{w}_{2}^{*}=\frac{\left(3 \delta^{2} v+2 c \mu \sigma_{s}^{2}\right) k}{2\left(5 \delta^{2} \hat{b}+2 c \hat{b} \mu \sigma_{s}^{2}+2 \hat{a} c\right)}$

(a) Optimal sales commission rate comparison

a-1) To show $w_{11}^{*}>\bar{w}_{1}^{*}$ is true, I should show that $\frac{4 \delta^{2} b-2 \delta \hat{b}+\hat{a} c}{2\left(4 \delta^{2} \hat{b}+c \hat{b} \mu \sigma_{s}^{2}+\hat{a} c\right)}-\frac{5 \delta^{2} \hat{b}-3 \delta v \hat{b}+2 \hat{a} c}{2\left(5 \delta^{2} \hat{b}+2 c \hat{b} \mu \sigma_{s}^{2}+2 \hat{a} c\right)}>$ 0. The left hand of this equation can be rewritten as below:

$$
2 \delta^{2} v \hat{b}+3 \delta \hat{b} c \mu \sigma_{s}^{2}-v c\left(\hat{a}+\hat{b} \mu \sigma_{s}^{2}\right)
$$

The equation 6.14 is larger than 0 in this case. Since the valid root of the above quadratic equation 6.14 is $\check{\delta}=\frac{-3 \hat{b} c \mu \sigma_{s}^{2}+\sqrt{8 b c\left(\hat{a}+\hat{b} \mu \sigma_{s}^{2}\right) v^{2}+\left(3 \hat{b} c \mu \sigma_{s}^{2}\right)^{2}}}{4 b v}$ and $\delta>0$, I can conclude that

$\left\{\begin{array}{l}w_{11}^{*} \leq \bar{w}_{1}^{*}, \text { if } \delta \leq \check{\delta}, \\ w_{11}^{*}>\bar{w}_{1}^{*}, \text { if } \delta>\check{\delta}\end{array}\right.$

a-2) Similarly, to show $\bar{w}_{1}^{*}>w_{21}^{*}$, I will prove $\frac{5 \delta^{2} \hat{b}-3 \delta v \hat{b}+2 \hat{a} c}{2\left(5 \delta^{2} \hat{b}+2 c \hat{b} \mu \sigma_{s}^{2}+2 \hat{a} c\right)}-\frac{\delta^{2} \hat{b}-\delta v \hat{b}+a c}{2\left(\delta^{2} \hat{b}+c \hat{b} \mu \sigma_{s}^{2}+\hat{a} c\right)}>0$. Then, by solving the fractional equation on the left side, I can drive the same equation 6.14. Hence, it has the same root with above a-1. Following that, I can conclude that $\left\{\begin{array}{l}\bar{w}_{1}^{*} \leq w_{21}^{*}, \text { if } \delta \leq \check{\delta}, \\ \bar{w}_{1}^{*}>w_{21}^{*}, \text { if } \delta>\check{\delta}\end{array}\right.$

(b) Optimal activity commission rate comparison

b-1) To show $w_{12}^{*}>\bar{w}_{2}^{*}$ is true, I should show $\frac{\left(2 \delta^{2} v+c \mu \sigma_{s}^{2}\right) k}{2\left(4 \delta^{2} \hat{b}+c \hat{b} \mu \sigma_{s}^{2}+\hat{a} c\right)}-\frac{\left(3 \delta^{2} v+2 c \mu \sigma_{s}^{2}\right) k}{2\left(5 \delta^{2} \hat{b}+2 c \hat{b} \mu \sigma_{s}^{2}+2 \hat{a} c\right)}>0$. The left hand of this equation is also equal to 6.14. Since equation 6.14 is larger than 
0 in this case, I can conclude that $\left\{\begin{array}{l}w_{21}^{*} \geq \bar{w}_{2}^{*} \text {, if } \delta \leq \check{\delta}, \\ w_{21}^{*}<\bar{w}_{2}^{*} \text {, if } \delta>\check{\delta}\end{array}\right.$

b-2) Similarly, $w_{22}^{*}>\bar{w}_{2}^{*}$ is equivalent to $\frac{\left(\delta^{2} v+c \mu \sigma_{s}^{2}\right) k}{2\left(\delta^{2} \hat{b}+c \hat{b} \mu \sigma_{s}^{2}+\hat{a} c\right)}-\frac{\left(3 \delta^{2} v+2 c \mu \sigma_{s}^{2}\right) k}{2\left(5 \delta^{2} \hat{b}+2 c \hat{b} \mu \sigma_{s}^{2}+2 \hat{a} c\right)}>0$.

Then, the left hand of this equation is same as equation 6.14 .

Therefore, $\left\{\begin{array}{l}w_{22}^{*} \leq \bar{w}_{2}^{*}, \text { if } \delta \leq \check{\delta}, \\ w_{22}^{*}>\bar{w}_{2}^{*}, \text { if } \delta>\check{\delta}\end{array}\right.$

By a-1), a-2), b-1), and b-2),

If $\delta \leq \check{\delta},\left\{\begin{array}{l}w_{11}^{*} \geq \bar{w}_{1}^{*} \geq w_{21}^{*}, \\ w_{22}^{*} \leq \bar{w}_{2}^{*} \leq w_{21}^{*},\end{array} \quad\right.$ If $\delta>\check{\delta},\left\{\begin{array}{l}w_{11}^{*}<\bar{w}_{1}^{*}<w_{21}^{*}, \\ w_{22}^{*}>\bar{w}_{2}^{*}>w_{21}^{*},\end{array}\right.$

(c) Optimal selling effort comparison

Under MD-C plan, selling effort amounts are as follows respectively;

$\left\{\begin{array}{l}\text { High base ability rep; } x_{1}^{*}=\frac{2 \delta}{c} w_{11}^{*}=\frac{2 \delta}{c} \frac{4 \delta^{2} \hat{b}-2 \delta v \hat{b}+\hat{a} c}{2\left(4 \delta^{2} \hat{b}+c \hat{b} \mu \sigma_{s}^{2}+\hat{a} c\right)} \\ \text { Low base ability rep; } x_{2}^{*}=\frac{\delta}{c} w_{21}^{*}=\frac{\delta}{c} \frac{\delta^{2} \hat{b}-\delta v \hat{b}+\hat{a} c}{2\left(\delta^{2} \hat{b}+c \hat{b} \mu \sigma_{s}^{2}+\hat{a} c\right)}\end{array}\right.$

Under MD-U plan, selling effort amounts are as follows respectively;

$\left\{\begin{array}{l}\text { High base ability rep; } \bar{x}_{1}^{*}=\frac{2 \delta}{c} \bar{w}_{1}^{*}=\frac{2 \delta}{c} \frac{5 \delta^{2} \hat{b}-3 \delta v \hat{b}+2 \hat{a} c}{2\left(5 \delta^{2} \hat{b}+2 c \hat{b} \mu \sigma_{s}^{2}+2 \hat{a} c\right)}, \\ \text { Low base ability rep; } \bar{x}_{2}^{*}=\frac{\delta}{c} \bar{w}_{1}^{*}=\frac{\delta}{c} \frac{5 \delta^{2} \hat{b}-3 \delta v \hat{b}+2 \hat{a} c}{2\left(5 \delta^{2} \hat{b}+2 c \hat{b} \mu \sigma_{s}^{2}+2 \hat{a} c\right)}\end{array}\right.$

Let $\sum x_{m c}^{*}=x_{1}^{*}+x_{2}^{*}$ and $\sum \bar{x}_{m u}^{*}=\bar{x}_{1}^{*}+\bar{x}_{2}^{*}$ are the firm's total selling efforts from the two reps under $\mathrm{MD}-\mathrm{C}$ and $\mathrm{MD}-\mathrm{U}$ plan respectively. Then, the goal is to show $\sum x_{m c}^{*} \gtreqless \sum \bar{x}_{m u}^{*}$.

Since $\sum x_{m c}^{*}-\sum \bar{x}_{m u}^{*}=\left(x_{1}^{*}+x_{2}^{*}\right)-\left(\bar{x}_{1}^{*}+\bar{x}_{2}^{*}\right)$, I should know if 1) $\left.x_{1}^{*}<\bar{x}_{1}^{*}, 2\right) x_{2}^{*}<\bar{x}_{2}^{*}$, and 3) $\left|x_{1}^{*}-\bar{x}_{1}^{*}\right|<\left|x_{2}^{*}-\bar{x}_{2}^{*}\right|$.

c-1) Show if $x_{1}^{*}<\bar{x}_{1}^{*}$ and $x_{2}^{*}<\bar{x}_{2}^{*}$ are true. 
$x_{1}^{*}<\bar{x}_{1}^{*}$ can be represented as $\frac{2 \delta}{2 c}\left(\frac{4 \delta^{2} \hat{b}-2 \delta v \hat{b}+\hat{a} c}{2\left(4 \delta^{2} \hat{b}+c \hat{b} \mu \sigma_{s}^{2}+\hat{a} c\right)}-\frac{5 \delta^{2} \hat{b}-3 \delta \hat{b}+2 \hat{a} c}{2\left(5 \delta^{2} \hat{b}+2 c \hat{c} \mu \sigma_{s}^{2}+2 \hat{a} c\right)}\right)<0$ and its left hand is equal to equation 6.14. Also, $x_{2}^{*}<\bar{x}_{2}^{*}$ is same with

$\frac{\delta}{2 c}\left(\frac{\delta^{2} \hat{b}-\delta v \hat{b}+\hat{a} c}{2\left(\delta^{2} \hat{b}+c \hat{c} \mu \sigma_{s}^{2}+\hat{a} c\right)}-\frac{5 \delta^{2} \hat{b}-3 \delta v \hat{b}+2 \hat{a} c}{2\left(5 \delta^{2} \hat{b}+2 c \hat{b} \mu \sigma_{s}^{2}+2 \hat{a} c\right)}\right)<0$ which is equal to equation 6.14 . Since the

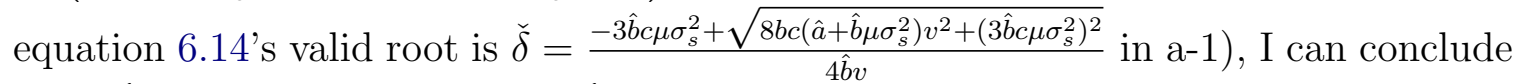
that $\left\{\begin{array}{ll}x_{1}^{*} \leq \bar{x}_{1}^{*}, & \text { if } \delta \leq \check{\delta} \\ x_{1}^{*}>\bar{x}_{1}^{*}, & \text { if } \delta>\check{\delta}\end{array}\right.$ and $\begin{cases}x_{2}^{*} \geq \bar{x}_{2}^{*}, & \text { if } \delta \leq \check{\delta} \\ x_{2}^{*}<\bar{x}_{2}^{*}, & \text { if } \delta>\check{\delta}\end{cases}$

c-2) Show if $\left|x_{1}^{*}-\bar{x}_{1}^{*}\right|<\left|x_{2}^{*}-\bar{x}_{2}^{*}\right|$ is true.

$\left|x_{1}^{*}-\bar{x}_{1}^{*}\right|<\left|x_{2}^{*}-\bar{x}_{2}^{*}\right|$ can be shown as $\frac{2 \delta}{2 c}\left(\frac{4 \delta^{2} \hat{b}-2 \delta \hat{b}+\hat{a} c}{2\left(4 \delta^{2} \hat{b}+c \hat{b} \mu \sigma_{s}^{2}+\hat{a} c\right)}-\frac{5 \delta^{2} \hat{b}-3 \delta v \hat{b}+2 \hat{a} c}{2\left(5 \delta^{2} \hat{b}+2 \hat{b} \hat{b} \mu \sigma_{s}^{2}+2 \hat{a} c\right)}\right)-\frac{\delta}{2 c}\left(\frac{\delta^{2} \hat{b}-\delta v \hat{v}+\hat{a} c}{2\left(\delta^{2} \hat{b}+c \hat{b} \mu \sigma_{s}^{2}+\hat{a} c\right)}-\frac{5 \delta^{2} \hat{b}-3 \delta \hat{b} \hat{b}+2 \hat{a} c}{2\left(5 \delta^{2} \hat{b}+2 c \hat{b} \mu \sigma_{s}^{2}+2 \hat{a} c\right)}\right)>0$. By solving the left side, I can derive $-2 \delta^{2} \hat{b}+c\left(\hat{a}+\hat{b} \mu \sigma_{s}^{2}\right)>0$.

By solving this quadratic equation, I will find the valid $\delta$ range as below; $\begin{cases}\left|x_{1}^{*}-\bar{x}_{1}^{*}\right| \geq\left|x_{2}^{*}-\bar{x}_{2}^{*}\right|, \quad \text { if } \delta \leq \sqrt{\frac{c\left(\hat{a}+\hat{b} \mu \sigma_{s}^{2}\right)}{2 b}} \\ \left|x_{1}^{*}-\bar{x}_{1}^{*}\right|<\left|x_{2}^{*}-\bar{x}_{2}^{*}\right|, \quad \text { if } \delta>\sqrt{\frac{c\left(\hat{a}+\hat{b} \mu \sigma_{s}^{2}\right)}{2 b}}\end{cases}$ Note that $\sqrt{\frac{c\left(\hat{a}+\hat{b} \mu \sigma_{s}^{2}\right)}{2 \hat{b}}}>\check{\delta}$. Then, by c-1), c-2), and $\sqrt{\frac{c\left(\hat{a}+\hat{b} \mu \sigma_{s}^{2}\right)}{2 \tilde{b}}}>\check{\delta}$, I can find that $\begin{cases}\sum x_{m c}^{*}>\sum \bar{x}_{m u}^{*}, & \text { if } \check{\delta}<\delta<\sqrt{\frac{c\left(\hat{a}+\hat{b} \mu \sigma_{s}^{2}\right)}{2 \hat{b}}} \\ \sum x_{m c}^{*}<\sum \bar{x}_{m u}^{*}, & \text { otherwise. }\end{cases}$

(d) Optimal thinking efforts

Let $\sum e_{m c}^{*}=\sum e_{1}^{*}+\sum e_{2}^{*}$ and $\sum \bar{e}_{m u}^{*}=\sum \bar{e}_{1}^{*}+\sum \bar{e}_{2}^{*}$ are the firm's total thinking efforts from the two reps under MD-C and MD-U plan respectively. Now the goal is to show $\sum e_{m c}^{*} \gtreqless \sum \bar{e}_{m u}^{*}$.

Since $\sum e_{m c}^{*}-\sum \bar{e}_{m u}^{*}=\left(\sum e_{1}^{*}+\sum e_{2}^{*}\right)-\left(\sum \bar{e}_{1}^{*}+\sum \bar{e}_{2}^{*}\right)$, I should know if 1) $\sum e_{1}^{*}<$ $\sum \bar{e}_{1}^{*}$, 2) $\sum e_{2}^{*}<\sum \bar{e}_{2}^{*}$, and 3) $\left|\sum e_{1}^{*}-\sum \bar{e}_{1}^{*}\right|<\left|\sum e_{2}^{*}-\sum \bar{e}_{2}^{*}\right|$.

Under MD-C plan, thinking effort amounts are as follows respectively; 
$\left\{\begin{array}{l}\text { High base ability rep; } \sum e_{1}^{*}=e_{11}^{*}+e_{12}^{*}=\frac{c a}{2 \delta c_{1} c_{2}}+\frac{c k}{2 \delta c_{1} c_{2}} \frac{w_{12}^{*}}{w_{11}^{*}} \\ \text { Low base ability rep; } \sum e_{2}^{*}=e_{11}^{*}+e_{12}^{*}=\frac{c a}{\delta c_{1} c_{2}}+\frac{c k}{\delta c_{1} c_{2}} \frac{w_{22}^{*}}{w_{21}^{*}}\end{array}\right.$ where $a=u^{2} c_{2}+\phi^{2} c_{1}$ and $k=u \gamma_{1} c_{2}+\phi \gamma_{2} c_{1}$.

Under MD-U plan, thinking effort amounts are as follows respectively;

$\left\{\begin{array}{l}\text { High base ability rep; } \sum \bar{e}_{1}^{*}=\bar{e}_{11}^{*}+\bar{e}_{12}^{*}=\frac{c a}{2 \delta c_{1} c_{2}}+\frac{c k}{2 \delta c_{1} c_{2}} \frac{\bar{w}_{2}^{*}}{\bar{w}_{1}^{*}} \\ \text { Low base ability rep; } \sum \bar{e}_{2}^{*}=\bar{e}_{21}^{*}+\bar{e}_{22}^{*}=\frac{c a}{\delta c_{1} c_{2}}+\frac{c k}{\delta c_{1} c_{2}} \frac{\bar{w}_{2}^{*}}{\bar{w}_{1}^{*}}\end{array}\right.$

d-1) Show $\sum e_{1}^{*}-\sum \bar{e}_{1}^{*}>0$ for rep 1 and $\sum e_{2}^{*}-\sum \bar{e}_{2}^{*}>0$ for rep 2 .

$\sum e_{1}^{*}-\sum \bar{e}_{1}^{*}$ is equal to $\frac{c a}{2 \delta c_{1} c_{2}}+\frac{c k}{2 \delta c_{1} c_{2}} \frac{w_{12}^{*}}{w_{11}^{*}}-\left(\frac{c a}{2 \delta c_{1} c_{2}}+\frac{c k}{2 \delta c_{1} c_{2}} \frac{\bar{w}_{2}^{*}}{\bar{w}_{1}^{*}}\right)$. By solving this equation, it can be also rewritten as equation 6.14. Similarly, $\sum e_{2}^{*}-\sum \bar{e}_{2}^{*}>0$ is equal to equation 6.14 .

Hence, I can conclude that $\left\{\begin{array}{ll}\sum e_{1}^{*} \geq \sum \bar{e}_{1}^{*}, & \text { if } \delta \leq \check{\delta} \\ \sum e_{1}^{*}<\sum \bar{e}_{1}^{*}, & \text { if } \delta>\check{\delta}\end{array}\right.$ and $\begin{cases}\sum e_{2}^{*} \leq \sum \bar{e}_{2}^{*}, & \text { if } \delta \leq \check{\delta} \\ \sum e_{2}^{*}>\sum \bar{e}_{2}^{*}, & \text { if } \delta>\check{\delta}\end{cases}$

d-2) Then, I need to show if $\left|\sum e_{1}^{*}-\sum \bar{e}_{1}^{*}\right|<\left|\sum e_{2}^{*}-\sum \bar{e}_{2}^{*}\right|$ is true or not.

It can be represented as $\frac{1}{2 \delta c_{1} c_{2}}\left(\frac{w_{12}^{*}}{w_{11}^{*}}-\frac{\bar{w}_{2}^{*}}{\bar{w}_{1}^{*}}\right)-\frac{1}{\delta c_{1} c_{2}}\left(\frac{w_{22}^{*}}{w_{21}^{*}}-\frac{\bar{w}_{2}^{*}}{\bar{w}_{1}^{*}}\right)<0$. By simplifying it, I can derive it as $-7 \delta^{2} \hat{b}+3 \delta \hat{b}-2 \hat{a} c<0$ which equation is true for $\forall \delta$.

Hence, $\left|\sum e_{1}^{*}-\sum \bar{e}_{1}^{*}\right|<\left|\sum e_{2}^{*}-\sum \bar{e}_{2}^{*}\right|$ for $\forall \delta$.

By d-1) and d-2), $\begin{cases}\sum e_{m c}^{*}>\sum \bar{e}_{m u}^{*}, & \text { if } \delta>\check{\delta} \\ \sum e_{m c}^{*}<\sum \bar{e}_{m u}^{*}, & \text { otherwise. }\end{cases}$

(e) Optimal sales comparison

Let $\sum s_{m c}^{*}=s_{1}^{*}+s_{2}^{*}$ and $\sum \bar{s}_{m u}^{*}=\bar{s}_{1}^{*}+\bar{s}_{2}^{*}$ are the firm's total sales from the two reps under MD-C and MD-U plan respectively. Show $\sum s_{m c}^{*} \gtreqless \sum \bar{s}_{m u}^{*}$.

Under MD-C plan; $\sum s_{m c}^{*}=2 \alpha+\frac{2 \delta}{c} w_{11}^{*} \beta_{1}^{*}+\frac{\delta}{c} w_{21}^{*} \beta_{2}^{*}$

Under MD-U plan; $\sum \bar{s}_{m u}^{*}=2 \alpha+\frac{2 \delta}{c} \bar{w}_{1}^{*} \bar{\beta}_{1}^{*}+\frac{\delta}{c} \bar{w}_{1}^{*} \bar{\beta}_{2}^{*}$

Let's show if $\sum s_{m c}^{*}<\sum \bar{s}_{m u}^{*}$. 
$\sum s_{m c}^{*}<\sum \bar{s}_{m u}^{*}$ can be rewritten as $2 \alpha+\frac{\delta}{c}\left(2 w_{11}^{*} \beta_{1}^{*}+w_{21}^{*} \beta_{2}^{*}\right)-2 \alpha+\frac{\delta}{c}\left(2 \bar{w}_{1}^{*} \bar{\beta}_{1}^{*}+\bar{w}_{1}^{*} \bar{\beta}_{2}^{*}\right)<$ 0 , and the left hand of the equation can be simplified as below;

$$
\left(4 \delta^{2} c_{1} c_{2}+c a\right)\left(w_{11}^{*}-\bar{w}_{1}^{*}\right)-c k^{2}\left(w_{11}^{*}-\bar{w}_{1}^{*}\right)-\left(\delta^{2} c_{1} c_{2}+c a\right)\left(w_{21}^{*}-\bar{w}_{1}^{*}\right)+c k^{2}\left(w_{21}^{*}-\bar{w}_{1}^{*}\right)
$$

where $a=\phi^{2} c_{1}+u^{2} c_{2}$ and $k=\phi \gamma_{2} c_{1}+u \gamma_{1} c_{2}$. Then, the sign of equation 6.15 is depending on the range of $\delta$.

If $\delta>\check{\delta}$, the equation 6.15 will be shown as follows;

$$
c_{1} c_{2}\left(\hat{a}+\hat{b} \mu \sigma_{s}^{2}\right)-\hat{b}\left(u^{2} c_{2}+\phi^{2} c_{1}-k^{2}\right)<0
$$

since $\left|w_{11}^{*}-\bar{w}_{1}^{*}\right|=\frac{2 \delta^{2} v \hat{b}+3 \delta \hat{b} c \mu \sigma_{s}^{2}-v c\left(\hat{a}+\hat{b} \mu \sigma_{s}^{2}\right)}{2\left(4 \delta^{2} \hat{b}+c \hat{b} \mu \sigma_{s}^{2}+\hat{a} c\right)\left(5 \delta^{2} \hat{b}+2 c \hat{b} \mu \sigma_{s}^{2}+2 \hat{a} c\right)}$ and $\left|w_{21}^{*}-\bar{w}_{1}^{*}\right|=\frac{2 \delta^{2} v \hat{b}+3 \delta \hat{b} c \mu \sigma_{s}^{2}-v c\left(\hat{a}+\hat{b} \mu \sigma_{s}^{2}\right)}{2\left(\delta^{2} \hat{b}+c \hat{b} \mu \sigma_{s}^{2}+\hat{a} c\right)\left(5 \delta^{2} \hat{b}+2 c \hat{b} \mu \sigma_{s}^{2}+2 \hat{a} c\right)}$. The equation 6.16 is false when $1<\gamma_{1}^{2} c_{2}$, $1<\gamma_{2}^{2} c_{1}\left(\because a-k^{2}=u^{2} c_{2}\left(1-\gamma_{1}^{2} c_{2}\right)+\phi^{2} c_{1}\left(1-\gamma_{2}^{2} c_{1}\right)-2 c_{1} c_{2} \gamma_{1} \gamma_{2} u \phi\right)$. Hence, if $\delta>\check{\delta}$, $1<\gamma_{1}^{2} c_{2}, 1<\gamma_{2}^{2} c_{1}$, then $\sum s_{m c}^{*}>\sum \bar{s}_{m u}^{*}$.

If $\delta \leq \check{\delta}$, the equation 6.15 will be as follows;

$$
-c_{1} c_{2}\left(\hat{a}+\hat{b} \mu \sigma_{s}^{2}\right)+\hat{b}(a-1)<0
$$

Equation 6.17 is true when $u^{2} c_{2}+\phi^{2} c_{1}<1$. Hence, if $\delta \leq \check{\delta}, 1<\gamma_{1}^{2} c_{2}, 1<\gamma_{2}^{2} c_{1}$, then $\sum s_{m c}^{*} \leq \sum \bar{s}_{m u}^{*}$.

Therefore, when $1<\gamma_{1}^{2} c_{2}, 1<\gamma_{2}^{2} c_{1}, \begin{cases}\sum s_{m c}^{*} \leq \sum \bar{s}_{m u}^{*}, & \text { if } \delta \leq \check{\delta} \\ \sum s_{m c}^{*}>\sum \bar{s}_{m u}^{*}, & \text { if } \delta>\check{\delta}\end{cases}$ 


\subsection{Proposition Proofs of Essay 2}

\subsubsection{Proof of Proposition 8}

Proof. (a) ISR's optimal level of selling effort regarding incentive rate is $e_{i o s}=$ $\frac{w_{2} v_{3} \beta_{i o s} \beta_{o q}^{2}}{c_{o q} c_{i o s}}$ and OSR's is $e_{o i s}=\frac{v_{2} w_{3} \beta_{o i s} \beta_{i q}^{2}}{c_{i q} c_{o i s}}$. By taking partial derivative, we confirm it's positive as follow;

$\frac{\partial e_{i o s}^{*}}{\partial v_{3}}=\frac{w_{2} \beta_{i o s} \beta_{o q}^{2}}{c_{o q} c_{i o s}}>0$ and $\frac{\partial e_{o i s}^{*}}{\partial w_{3}}=\frac{v_{2} \beta_{o i s} \beta_{i q}^{2}}{c_{i q} c_{o i s}}>0$.

(b) ISR's optimal level of dishonest report is $\Omega_{i}=w_{3}\left(\frac{1}{2 w_{4}}-\mu \sigma_{i 2}^{2}\right)$ and OSR's is $\Omega_{o}=v_{3}\left(\frac{1}{2 v_{4}}-\rho \sigma_{o 2}^{2}\right)$. By taking partial derivative, we confirm it's negative as follow; $\frac{\partial \Omega_{i}^{*}}{\partial w_{4}}=-\frac{w_{3}}{2 w_{4}^{2}}<0$ and $\frac{\partial \Omega_{o}^{*}}{\partial v_{4}}=-\frac{v_{3}}{2 v_{4}^{2}}<0$.

None of the selling efforts $\left(e_{i i s}^{*}, e_{o o s}^{*}, e_{i o s}^{*}, e_{o i s}^{*}\right)$ are not functions of $w_{4}$ or $w_{4}$.

\subsubsection{Proof of Proposition 9}

Proof. (a) ISR's optimal incentive on cooperative selling is $w_{2}^{*}=\frac{\beta_{i o s} \beta_{o q}-\sqrt{\beta_{i o s}^{2} \beta_{o q}^{2}-c_{i o s} c_{o q}}}{\beta_{i o s} \beta_{o q}}$ which is not a function of reps' risk aversion $(\mu, \rho)$ nor market uncertainties from any side $\left(\sigma_{i 2}^{2}, \sigma_{o 2}^{2}\right)$. It's vice versa for OSR.

(b) ISR's optimal incentive on cooperative qualifying leads is $w_{3}^{*}=\frac{\sigma_{o 2} c_{i q}}{\beta_{o i s} \beta_{i q}^{2}} \sqrt{\frac{2 c_{i o s} \mu\left(\beta_{i o s} \beta_{o q}-\sqrt{\beta_{i o s}^{2} \beta_{o q}^{2}-c_{i o s} c_{o q}}\right)}{\sqrt{\beta_{i o s}^{2} \beta_{o q}^{2}-c_{i o s} c_{o q}}}}$. By taking partial derivative, we confirm it's positive as follow;

$$
\frac{\partial w_{3}^{*}}{\partial \rho}=\frac{\sigma_{o 2} c_{i q}}{\beta_{o i s} \beta_{i q}^{2}} \sqrt{\frac{\sqrt{\beta_{i o s}^{2} \beta_{o q}^{2}-c_{i o s} c_{o q}}}{2 c_{i o s} \mu\left(\beta_{i o s} \beta_{o q}-\sqrt{\beta_{i o s}^{2} \beta_{o q}^{2}-c_{i o s} c_{o q}}\right)}}>0
$$

and

$$
\frac{\partial w_{3}^{*}}{\partial \sigma_{o 2}}=\frac{c_{i q}}{\beta_{o i s} \beta_{i q}^{2}} \sqrt{\frac{2 c_{i o s} \mu\left(\beta_{i o s} \beta_{o q}-\sqrt{\beta_{i o s}^{2} \beta_{o q}^{2}-c_{i o s} c_{o q}}\right)}{\sqrt{\beta_{i o s}^{2} \beta_{o q}^{2}-c_{i o s} c_{o q}}}}>0 .
$$




\subsubsection{Proof of Proposition 10}

Proof. (a) The optimal penalty rates for ISR's and OSR's dishonest report are respectively $w_{4}^{*}=\frac{1}{2 \mu \sigma_{i 2}^{2}}, v_{4}^{*}=\frac{1}{2 \rho \sigma_{o 2}^{2}}$. By taking partial derivative, we confirm it's negative as follow;

$\frac{\partial w_{4}^{*}}{\partial \mu}=-\frac{1}{2 \mu^{2} \sigma_{i 2}^{2}}<0$ and $\frac{\partial w_{4}^{*}}{\partial \sigma_{i 2}}=\frac{1}{\mu \sigma_{i 2}^{3}}<0$.

(b) ISR's optimal level of dishonest report is $\Omega_{i}^{*}=\frac{\sigma_{o 2} c_{i q}}{\beta_{o i s} \beta_{i q}^{2}}\left(\mu \sigma_{i 2}^{2}-\mu \sigma_{i 2}^{2}\right)=0$ and OSR's is $\Omega_{o}^{*}=\frac{\sigma_{i 2} c_{o q}}{\beta_{i o s} \beta_{o q}^{2}}\left(\rho \sigma_{o 2}^{2}-\rho \sigma_{o 2}^{2}\right)=0$.

\subsubsection{Proof of Proposition 11}

Proof. (a) ISR's optimal cooperative selling effort is $e_{i o s}^{*}=\frac{\sigma_{i 2}\left(\beta_{i o s} \beta_{o q}-\sqrt{\beta_{i o s}^{2} \beta_{o q}^{2}-c_{i o s} c_{o q}}\right)}{p_{i} \beta_{i o s} \beta_{o q} c_{i o s}} \sqrt{\frac{2 c_{i o s} \mu\left(\beta_{i o s} \beta_{o q}-\sqrt{\beta_{i o s}^{2} \beta_{o q}^{2}-c_{i o s} c_{o q}}\right)}{\sqrt{\beta_{i o s}^{2} \beta_{o q}^{2}-c_{i o s} c_{o q}}}}$. By taking derivative, $\frac{\partial e_{i o s}^{*}}{\partial \beta_{i o s}}>0$ because $\frac{\partial v_{3}^{*}}{\partial \beta_{i o s}}>0$. Vise versa for OSR's optimal cooperative selling effort.

By taking derivative, $\frac{\partial e_{i o s}^{*}}{\partial \beta_{o q}}=\frac{\mu \sigma_{i 2}\left(\beta_{i o s} \beta_{o q}-\sqrt{\beta_{i o s}^{2} \beta_{o q}^{2}-c_{i o s} c_{o q}}\right) H}{\left.p_{i} \beta_{o q}^{2} \beta_{i o s} \sqrt{\beta_{i o s}^{2} \beta_{o q}^{2}-c_{i o s} c_{o q}} \sqrt{2 \mu c_{i o s}\left(\beta_{i o s} \beta_{o q}-\sqrt{\beta_{i o s}^{2} \beta_{o q}^{2}-c_{i o s} c_{o q}}\right.}\right)}>$ 0 where $H=\beta_{i o s} \beta_{o q} \sqrt{\beta_{i o s}^{2} \beta_{o q}^{2}-c_{i o s} c_{o q}}-\beta_{i o s}^{2} \beta_{o q}^{2}-2 c_{i o s} c_{o q}$.

(b) ISR's optimal cooperative qualifying effort is

$e_{i q}^{*}=\frac{\sigma_{o 2}}{p_{o} \beta_{i q} \beta_{o i s}} \sqrt{\frac{2 c_{o i s} \rho\left(\beta_{o i s} \beta_{i q}-\sqrt{\beta_{o i s}^{2} \beta_{i q}^{2}-c_{o i s} c_{i q}}\right)}{\sqrt{\beta_{o i s}^{2} \beta_{i q}^{2}-c_{o i s} c_{i q}}}}$.

By taking derivative, $\frac{\partial e_{i q}^{*}}{\partial \beta_{i q}}=-\frac{c_{o i s} \rho \sigma_{o 2}\left(\beta_{o i s} \beta_{i q} \sqrt{\beta_{o i s}^{2} \beta_{i q}^{2}-c_{o i s} c_{i q}}-\beta_{o i s}^{2} \beta_{i q}^{2}+2 c_{o i s} c_{i q}\right)}{p_{o} \beta_{i q}^{2} \beta_{o i s} \sqrt{\beta_{o i s}^{2} \beta_{i q}^{2}-c_{o i s} c_{i q}}}<0$.

Also, $\frac{\partial e_{i q}^{*}}{\partial \beta_{o i s}}>0$ because $\frac{\partial v_{3}^{*}}{\partial \beta_{i o s}}>0$. 


\section{Web Appendix}

\subsection{Comparative Statics Proofs for Essay 1}

\subsubsection{SC plan for Table 4}

From Table 3, $w_{1 j}^{*}=\frac{\delta_{j} c_{1 j} c_{2 j}\left(\delta_{j}-v\right)+c_{j}\left(c_{j 1} \phi_{j}^{2}+c_{2 j} u_{j}^{2}\right)}{2\left(\left(c_{j} c_{1 j} c_{2 j} \mu_{j} \sigma_{S j}^{2}+c_{1 j} c_{2 j} \delta_{j}^{2}+c_{j}\left(c_{1 j} \phi_{j}^{2}+c_{2 j} u_{j}^{2}\right)\right)\right)}$.

1. Uncertainty $\sigma_{s j}$

Let $w_{1 j}^{*}=\frac{a}{2\left(b+c \sigma_{s j}\right)}$

1-1. $\frac{\partial w_{1 j}^{*}}{\partial \sigma_{s j}}=-\frac{a c \sigma_{s j}}{\left(b+c \sigma_{s j}^{2}\right)^{2}}<0$.

1-2. $\frac{\partial x_{j}^{*}}{\partial \sigma_{s j}}=-\frac{g a c \sigma_{s j}}{\left(b+c \sigma_{s j}^{2}\right)^{2}}<0$ where $g=\frac{\delta_{j}}{c_{j}}$.

1-3. $\frac{\partial \beta_{j}^{*}}{\partial \sigma_{s j}}=0$ because $e_{1 j}, e_{2 j}, \beta_{j}$ are not functions of $\sigma_{s j}$.

1-4. $\frac{\partial E(s)}{\partial \sigma_{s j}}=-\frac{g a c \beta \sigma_{s j}}{\left(b+c \sigma_{s j}^{2}\right)^{2}}<0$

1-5. $\frac{\partial E(w)}{\partial \sigma_{s j}} \approx \frac{\partial\left(g \beta w_{1}^{* 2}\right)}{\partial \sigma_{s j}}=-\frac{g a^{2} c \beta \sigma_{s j}}{\left(b+c \sigma_{s j}^{2}\right)^{3}}<0$. Hence, $\frac{\partial \frac{w_{0}}{E(w)}}{\partial \sigma_{s j}}>0$.

1-6. $\frac{\partial \pi_{j}}{\partial \sigma_{s j}} \approx \frac{\partial\left(g(\beta-\nu) w_{1}^{*}-g \beta w_{1}^{* 2}\right)}{\partial \sigma_{s j}}=-\frac{g a c(\beta-\nu) \sigma_{s j}}{\left(b+c \sigma_{s j}^{2}\right)^{2}}+\frac{g a^{2} c \beta \sigma_{s j}}{\left(b+c \sigma_{s j}^{2}\right)^{3}}<0$.

2. Risk aversion $\mu_{j}$

Let $w_{1 j}^{*}=\frac{a}{2(b+c \mu)}$.

2-1. $\frac{\partial w_{1 j}^{*}}{\partial \mu_{j}}=-\frac{a c}{2(b+c \mu)^{2}}<0$.

2-2. $\frac{\partial e_{1 j}^{*}}{\partial \mu_{j}}=\frac{\partial e_{2 j}^{*}}{\partial \mu_{j}}=\frac{\partial \beta_{j}^{*}}{\partial \mu_{j}}=0$ since none of them are function of $\mu$.

2-3. $\frac{\partial x_{j}^{*}}{\partial \mu_{j}}=-\frac{a c g}{2(b+c \mu)^{2}}<0$ where $g=\frac{\delta_{j}}{c_{j}}$.

2-4. $\frac{\partial E(s)}{\partial \mu_{j}}=-\frac{a g c \beta_{j}^{*}}{2(b+c \mu)^{2}}<0$.

2-5. $\frac{\partial E(w)}{\partial \mu_{j}}=-\frac{a c(c \alpha \mu+a g \beta+b \alpha)}{2(b+c \mu)^{3}}<0$ where $\alpha, \beta$ are not functions of $\mu$.

2-6. $\frac{\partial \pi_{j}}{\partial \mu_{j}}=\frac{a g \beta}{2(b+c \mu)}-\frac{a \alpha}{2(b+c \mu)}-\left(\frac{a}{2(b+c \mu)^{2}}\right) g \beta-v g \frac{a}{2(b+c \mu)}<0 \frac{\partial \frac{w_{0}}{E(w)}}{\partial \mu_{j}}>0$ by 3-5.

3. Selling cost $c_{j}$

Let $w_{1 j}^{*}=\frac{a+c_{j} b}{2\left(c_{j} d+f+c_{j} b\right)}$ and $x_{j}^{*}=\frac{\delta_{j}(a+c b)}{2 c_{j}\left(c_{j} d+f+c_{j} b\right)}$.

3-1. $\frac{\partial w_{1 j}^{*}}{\partial c_{j}}=\frac{b f-a(b+d)}{2\left(c_{j}(b+d)+f\right)^{2}}$.

Since $b f-a(b+d)=\left(c_{1 j} \phi_{j}^{2}+c_{2 j} u_{j}^{2}\right) c_{1 j} c_{2 j} \sigma_{s j}^{2}-\delta_{j} c_{1 j} c_{2 j}\left(\delta_{j}-\nu\right)\left(c_{1 j} \phi_{j}^{2}+c_{2 j} u_{j}^{2}+c_{1 j} c_{2 j} \mu_{j} \sigma_{s j}^{2}\right)$, 
when $\sigma_{s j}^{2}-\frac{\delta_{j} c_{1 j} c_{2 j}\left(\delta_{j}-\nu\right)\left(c_{1 j} \phi_{j}^{2}+c_{2 j} u_{j}^{2}+c_{1 j} c_{2 j} \mu_{j} \sigma_{s j}^{2}\right.}{\left(c_{1 j} \phi_{j}^{2}+c_{2 j} u_{j}^{2}\right) c_{1 j} c_{2 j} \sigma_{s j}^{2}}>0, \frac{\partial w_{1 j}^{*}}{\partial c_{j}}>0$ and vise versa.

3-2. $\frac{\partial x_{j}^{*}}{\partial c_{j}}=-\frac{\delta_{j}\left(\left(b d+b^{2}\right) c_{j}^{2}+2(a d+2 a b) x_{j}+a f\right)}{2 c_{j}^{2}\left(c_{j}(d+f)+f\right)^{2}}<0$.

3-3. $\frac{\partial \beta_{j}^{*}}{\partial c_{j}}=\frac{u_{j}^{2}}{c_{1 j} \delta_{j}}+\frac{\phi_{j}^{2}}{c_{2 j} \delta_{j}}>0$.

3-4. $E(w)=w_{0}+w_{1 j}^{*} E(s)=w_{0}+w_{1 j}^{*} x_{j}^{*} \beta_{j}^{*}=w_{0}+w_{1 j}^{* 2} \frac{\delta_{j}}{c_{j}} \delta_{j}+c_{j} h$ where $h=$ $\frac{u_{j}^{2}}{c_{1 j} \delta_{j}}+\frac{\phi_{j}^{2}}{c_{2 j} \delta_{j}}$.

$\frac{\partial E(w)}{\partial c_{j}}=-\frac{\delta(b c+a)\left(2 b(b+d) h c^{3}+(b+d)(3 b \delta+4 a h) c^{2}+((b f+5 a d+5 a b) \delta+2 a f h) c+3 a f \delta\right)}{4 c^{4}((b+d) c+f)^{3}}<0$.

3-5. $\pi_{j}=E(s)-E(w)-L^{*}=x_{j}^{*} \beta_{j}^{*}-w_{0}-w_{1 j}^{*} x_{j}^{*} \beta_{j}^{*}-\nu x_{j}^{*}$. By taking derivative in mathematica, I confirm that $\frac{\partial\left(\left(\left(1-w_{1 j}^{*}\right) \beta_{j}^{*}-v_{j}\right) x_{j}^{*}-w_{o}\right)}{\partial c_{j}}=\frac{\partial \pi_{j}}{\partial c_{j}}<0$.

4. Thinking cost $c_{1 j}$ (similar to $c_{2 j}$ )

Let $w_{1 j}^{*}=\frac{\delta_{j} c_{1 j} c_{2 j}\left(\delta_{j}-v\right)+c_{j}\left(c_{j 1} \phi_{j}^{2}+c_{2 j} u_{j}^{2}\right)}{2\left(\left(c_{j} c_{1 j} c_{2 j} \mu_{j} \sigma_{S j}^{2}+c_{1 j} c_{2 j} \delta_{j}^{2}+c_{j}\left(c_{1 j} \phi_{j}^{2}+c_{2 j} u_{j}^{2}\right)\right)\right)}=\frac{a c_{1 j}+b c_{1 j}+d}{2\left(c_{1 j} f+c_{1 j} g+c_{1 j} b+d\right)}$.

4-1. $\frac{\partial w_{1 j}^{*}}{\partial c_{1 j}}=-\frac{d(g+f-a)}{2\left(c_{1 j}(g+f+b)+d\right)^{2}}<0$

4-2. $\frac{\partial x_{j}^{*}}{\partial c_{1 j}}=-\frac{d(g+f-a) \delta}{2 c\left(c_{1 j}(g+f+b)+d\right)^{2}}<0$

4-3. $\frac{\partial \beta_{j}^{*}}{\partial c_{1 j}}=-\frac{u^{2} c_{j}}{\delta_{j} c_{1 j}^{2}}<0$

4-4. $E(w)=w_{0}+w_{1 j}^{*} x_{j}^{*} \beta_{j}^{*}=w_{0}+w_{1 j}^{* 2}\left(s+\frac{t}{c_{1 j}}\right)$ where $s=\delta_{j}+\frac{\phi_{j} c_{j}}{\delta_{j} c_{2 j}}$ and $t=\frac{u_{j}^{2} c_{j}}{\delta_{j}}$. $\frac{\partial E(w)}{\partial c_{1 j}}<0$. Hence, $\frac{\partial \frac{w_{0}}{E(w)}}{\partial c_{1 j}}>0$.

4-5. $\frac{\partial \pi_{j}}{\partial c_{1 j}}$. By taking derivative in mathematica, I confirm that $\frac{\partial\left(\left(\left(1-w_{1 j}^{*}\right) \beta_{j}^{*}-v_{j}\right) x_{j}^{*}-w_{o}\right)}{\partial c_{1 j}}<$ 0.

5. Thinking effectiveness $u_{j}$ (similar to $\left.\phi_{j}\right)$

Let $w_{1 j}^{*}=\frac{\delta_{j} c_{1 j} c_{2 j}\left(\delta_{j}-v\right)+c_{j}\left(c_{j 1} \phi_{j}^{2}+c_{2 j} u_{j}^{2}\right)}{2\left(\left(c_{j} c_{1 j} c_{2 j} \mu_{j} \sigma_{S j}^{2}+c_{1 j} c_{2 j} \delta_{j}^{2}+c_{j}\left(c_{1 j} \phi_{j}^{2}+c_{2 j} u_{j}^{2}\right)\right)\right)}=\frac{a+b+c u_{j}^{2}}{2\left(d+f+b+c u_{j}^{2}\right)}$.

5-1. $\frac{\partial w_{1 j}^{*}}{\partial u_{j}}=\frac{c(f+d-a) u_{j}}{\left(c u_{j}^{2}+f+d+b\right)^{2}}>0$

5-2. $\frac{\partial \beta_{j}^{*}}{\partial u_{j}}=2 \frac{u_{j} c_{j}}{c_{1 j} \delta_{j}}>0$

5-3. $\frac{\partial x_{j}^{*}}{\partial u_{j}}=\frac{c(f+d-a) u_{j} s}{\left(c u_{j}^{2}+f+d+b\right)^{2}}>0$ where $s=\frac{\delta_{j}}{c_{j}}$.

5-4. $\frac{\partial E(s)}{\partial u_{j}}=\frac{\partial x_{j}^{*} \beta_{j}^{*}}{\partial u_{j}}>0$ by $5-1$ and 5-3.

5-5. $\frac{\partial E(w)}{\partial u_{j}}=\frac{w_{0}+w_{1}^{*} \partial E(s)}{\partial u_{j}}>0$ by 5-4 and 5-1 above. Hence, $\frac{\partial \frac{w_{0}}{E(w)}}{\partial u_{j}}<0$.

5-6. $\frac{\partial \pi_{j}}{\partial u_{j}}>0$. By taking derivative in mathematica, I confirm that 
$\frac{\partial\left(\left(\left(1-w_{1 j}^{*}\right) \beta_{j}^{*}-v_{j}\right) x_{j}^{*}-w_{o}\right)}{\partial u_{j}}<0$.

6. Basic ability $\delta_{j}$

Let $w_{1 j}^{*}=\frac{\delta_{j} c_{1 j} c_{2 j}\left(\delta_{j}-v\right)+c_{j}\left(c_{j 1} \phi_{j}^{2}+c_{2 j} u_{j}^{2}\right)}{2\left(\left(c_{j} c_{1 j} c_{2 j} \mu_{j} \sigma_{S j}^{2}+c_{1 j} c_{2 j} \delta_{j}^{2}+c_{j}\left(c_{1 j} \phi_{j}^{2}+c_{2 j} u_{j}^{2}\right)\right)\right)}=\frac{a \delta^{2}-a v \delta+f}{2\left(a \delta^{2} d\right)}$.

6-1. $\frac{\partial w_{1 j}^{*}}{\partial \delta_{j}}=\frac{a\left(a v \delta^{2}+2(d-f) \delta-d v\right.}{2\left(a \delta^{2} d\right)^{2}} \gtrless 0$.

6-2. $\frac{\partial e_{1 j}^{*}}{\partial \delta_{j}}=\frac{c u}{c_{1} \delta^{2}}<0$. Also, $\frac{\partial e_{2 j}^{*}}{\partial \delta_{j}}=\frac{c \phi}{c_{2} \delta^{2}}<0$. Based on $\frac{\partial e_{1 j}^{*}}{\partial \delta_{j}}, \frac{\partial e_{2 j}^{*}}{\partial \delta_{j}}, \frac{\partial \beta_{j}^{*}}{\partial \delta_{j}}=1-$ $\frac{c\left(c_{2} \phi^{2}+c_{1} u^{2}\right)}{c_{1} c_{2} \delta^{2}} \gtrless 0$.

6-3. $\frac{\partial x_{j}^{*}}{\partial \delta_{j}}=\frac{a^{2} \delta^{4}+(3 a d-a f) \delta^{2}-2 a d v \delta+f d}{2 c\left(a \delta^{2}+d\right)^{2}}>0$.

6-4. $\frac{\partial E(s)}{\partial \delta_{j}}=\frac{(2 a \delta-a v)(g+o)}{2\left(a \delta^{2}+d\right)}-\frac{a \delta\left(a \delta^{2}-a v \delta+c\right)(g+o)}{\left(a \delta^{2}+d\right)^{2}}+\frac{\delta\left(a \delta^{2}-a v \delta+c\right)}{c\left(a \delta^{2}+d\right)} \gtrless 0$ where $g=\frac{\delta^{2}}{c}, o=$ $\frac{c_{2} u^{2}+c_{1} \phi^{2}}{c_{1} c_{2}}$.

6-5. $\frac{\partial E(w)}{\partial \delta_{j}} \gtrless 0$ by 6 -4 above. Hence, $\frac{\partial \frac{w_{0}}{E(w)}}{\partial \delta_{j}} \gtrless 0$.

6-6. $\frac{\partial \pi_{j}}{\partial \delta_{j}} \gtrless 0$ by $6-4$ above.

\subsubsection{MD-C plan for Table 5}

Note that $w_{m c, 1}^{*}=\frac{c_{j}\left[\left(\phi_{j} \gamma_{1 j}-u_{j} \gamma_{2 j}\right)^{2}+\left(c_{1 j} \phi_{j}^{2}+c_{2 j} u_{j}^{2}\right) \mu_{j} \sigma_{K j}^{2}\right]+\delta(\delta-v)\left(\gamma_{1 j}^{2} c_{2 j}+\gamma_{2 j}^{2} c_{1 j}+\mu_{j} \sigma_{K j}^{2} c_{1 j} c_{2 j}\right)}{2\left(c_{j}\left[\left(\phi_{j} \gamma_{1 j}-u_{j} \gamma_{2 j}\right)^{2}+\left(c_{1 j} \phi_{j}^{2}+c_{2 j} u_{j}^{2}\right) \mu_{j} \sigma_{K j}^{2}\right]+\left(\delta_{j}^{2}+\mu_{j} \sigma_{S j}^{2} c_{j}\right)\left(\gamma_{1 j}^{2} c_{2 j}+\gamma_{2 j}^{2} c_{1 j}+\mu_{j} \sigma_{K j}^{2} c_{1 j} c_{2 j}\right)\right)}$, $w_{m c, 2}^{*}=\frac{\gamma_{2 j} c_{1 j} \phi_{j}+\gamma_{1 j} c_{2 j} u_{j}}{\gamma_{1 j}^{2} c_{2 j}+\gamma_{2 j}^{2} c_{1 j}+\mu_{j} \sigma_{K j}^{2} c_{1 j} c_{2 j}}\left(\frac{1}{2}-w_{m c, 1}^{*}\right)$, and $\frac{w_{m c, 2}^{*}}{w_{m c, 1}^{*}}=\frac{\gamma_{2 j} c_{1 j} \phi_{j}+\gamma_{1 j} c_{2 j} u_{j}}{\gamma_{1 j}^{2} c_{2 j}+\gamma_{2 j}^{2} c_{1 j}+\mu_{j} \sigma_{K j}^{2} c_{1 j} c_{2 j}}\left(\frac{1}{2 w_{m c, 1}^{*}}-\right.$ $1)$.

1. Uncertainty $\sigma_{s j}^{2}$

Let $w_{m c, 1}^{*}=\frac{a}{2\left(b+c \sigma_{s j}^{2}\right)}, w_{m c, 2}^{*}=d\left(\frac{1}{2}-\frac{a}{2\left(b+c \sigma_{s j}^{2}\right)}\right), \frac{w_{m c, 2}^{*}}{w_{m c, 1}^{*}}=d\left(\frac{\left(b+c \sigma_{s j}^{2}\right)}{a}-1\right)$.

1-1. $\frac{\partial w_{1 j}^{*}}{\partial \sigma_{s j}^{2}}=-\frac{a c \sigma_{s j}}{\left(b+c \sigma_{s j}^{2}\right)^{2}}<0 \quad, \frac{\partial w_{2 j}^{*}}{\partial \sigma_{s j}^{2}}=\frac{a c d \sigma_{s j}}{2\left(b+c \sigma_{s j}^{2}\right)^{2}}>0$, and $\frac{\partial \frac{w_{m c, 2}^{*}}{w_{m c, 1}^{*}}}{\partial \sigma_{s j}^{2}}=\frac{2 c d \sigma_{s j}}{a}>0$.

Hence, I can confirm that $\frac{\partial x_{j}^{*}}{\partial \sigma_{s j}^{2}}=-\frac{a c \delta \sigma_{s j}}{c\left(b+c \sigma_{s j}^{2}\right)^{2}}<0$.

1-2. $\frac{\partial e_{1 j}^{*}}{\partial \sigma_{s j}^{2}}>0, \frac{\partial e_{2 j}^{*}}{\partial \sigma_{s j}^{2}}>0$ by 1-1 above. Therefore, $\frac{\partial \beta_{j}^{*}}{\partial \sigma_{s j}^{2}}>0$.

1-3. $\frac{\partial E(s)}{\partial \sigma_{s j}^{2}}=-\frac{a c g \sigma_{s j}(d f-h)}{\left(b+c \sigma_{s j}^{2}\right.}<0$ where $h=\delta+u e+\phi k$ and $f=u q+i \phi$.

1-4. $\frac{\partial E(k)}{\partial \sigma_{s j}^{2}}=-\frac{a c g(m-d p) \sigma_{s j}}{\left(b+c \sigma_{s j}^{2}\right.}<0$ where $m=\frac{c u \gamma_{1}}{c_{1} \delta}+\frac{c \phi \gamma_{2}}{c_{2} \delta}$ and $p=\frac{c}{\delta}\left(\frac{\gamma_{1}^{2}}{c_{2}}+\frac{\gamma_{2}^{2}}{c_{1}}\right)$.

1-5. $\frac{\partial E(w)}{\partial \sigma_{s j}^{2}}<0$ by 1-1, 1-3, and 1-4 above. Hence, $\frac{\partial \frac{w_{0}}{E(w)}}{\partial \sigma_{s j}^{2}}>0$. 
1-6. $\frac{\partial \pi_{j}}{\partial \sigma_{s j}^{2}}=\frac{\partial\left(x^{*}\left(\beta^{*}-v\right)-w_{1}^{*} E\left(s^{*}\right)-w_{2}^{*} E\left(k^{*}\right)-w_{0}\right)}{\partial \sigma_{s j}^{2}}<0$ by 1-1, 1-2, and 1-5.

2. Uncertainty $\sigma_{k j}^{2}$

Let $w_{m c, 1}^{*}=\frac{a}{2\left(c+d \sigma_{k j}^{2}\right)}, w_{m c, 2}^{*}=\frac{f}{g+h \sigma_{k j}^{2}}\left(\frac{1}{2}-w_{m c, 1}^{*}\right), \frac{w_{m c, 2}^{*}}{w_{m c, 1}^{*}}=\frac{f}{g+h \sigma_{k j}^{2}}\left(\frac{\left(c+d \sigma_{k j}^{2}\right)}{a+b \sigma_{k j}^{2}}-1\right)$.

2-1. $\frac{\partial w_{1 j}^{*}}{\partial \sigma_{k j}^{2}}=-\frac{(a d-b c) \sigma_{k j}}{\left(c+d \sigma_{k j}^{2}\right)^{2}}<0 \quad, \frac{\partial w_{2 j}^{*}}{\partial \sigma_{s j}^{2}}=-\frac{f \sigma_{k j} z}{\left(c+d \sigma_{s j}^{2}\right)^{2}\left(g+h \sigma_{s j}^{2}\right)^{2}}<0$, and $\frac{\partial \frac{w_{m c, 2}^{*}}{w_{m c, 1}^{*}}}{\partial \sigma_{s j}^{2}}=$ $-\frac{2 f \sigma_{k j} z}{\left(a+b \sigma_{s j}^{2}\right)^{2}\left(g+h \sigma_{s j}^{2}\right)^{2}}<0$ where $z=\left(d^{2}-b d\right) h \sigma_{k j}^{4}+(c-a) h\left(2 d \sigma_{k j}^{2}+c\right)+g(b c-a d)$.

Hence, I can also confirm that $\frac{\partial x_{j}^{*}}{\partial \sigma_{k j}^{2}}=-\frac{(a d-b c) g \sigma_{k j}}{\left(c+d \sigma_{s j}^{2}\right)^{2}}<0$.

2-2. $\frac{\partial e_{1 j}^{*}}{\partial \sigma_{k j}^{2}}<0 \quad, \frac{\partial e_{2 j}^{*}}{\partial \sigma_{k j}^{2}}<0$ by $2-1$ above. Therefore, $\frac{\partial \beta_{j}^{*}}{\partial \sigma_{s j}^{2}}<0$.

2-3. $\frac{\partial E(s)}{\partial \sigma_{k j}^{2}}<0$ and $\frac{\partial E(k)}{\partial \sigma_{k j}^{2}}<0$. These are computed using mathematica.

2-4. $\frac{\partial E(w)}{\partial \sigma_{k j}^{2}}<0$ by $2-1$ and 2-4 above. Hence, $\frac{\partial \frac{w_{0}}{E(w)}}{\partial \sigma_{s j}^{2}}>0$.

2-5. $\frac{\partial \pi_{j}}{\partial \sigma_{k j}^{2}}<0$ by $2-1,2-2$, and $2-4$.

3. Risk aversion $\mu_{j}$

Let $w_{m c, 1}^{*}=\frac{a+b \mu}{2\left(c+d \mu+y \mu^{2}\right)}, w_{m c, 2}^{*}=\frac{f}{g+h \mu}\left(\frac{1}{2}-w_{m c, 1}^{*}\right), \frac{w_{m c, 2}^{*}}{w_{m c, 1}^{*}}=\frac{f}{g+h \mu}\left(\frac{\left(c+d \sigma_{k j}^{2}\right)}{a+b \sigma_{k j}^{2}}-1\right)$.

3-1. $\frac{\partial w_{1 j}^{*}}{\partial \mu_{j}}=-\frac{b y \mu^{2}+2 a y \mu+a d-b c}{2\left(c+d \mu+y \mu^{2}\right)^{2}}<0 \quad, \frac{\partial w_{2 j}^{*}}{\partial \mu_{j}}=\frac{f z}{2(g+h \mu)^{2}(c+(d+y \mu) \mu)^{2}}>0$ where $z=$ $a c h+a d(g+2 h \mu)+a y \mu(2 g+3 h \mu)-b c g+b \mu^{2}(d h+y(g+2 h \mu))-h(c+\mu(d+y \mu))^{2}$. Ergo, $\frac{\partial \frac{w_{m c, 2}^{*}}{w_{m c, 1}^{*}}}{\partial \sigma_{s j}^{2}}>0$ by computation. Therefore, I can also confirm that $\frac{\partial x_{j}^{*}}{\partial \mu_{j}}<0$.

3-2. $\frac{\partial e_{1 j}^{*}}{\partial \mu}>0, \frac{\partial e_{2 j}^{*}}{\partial \mu}>0$ by $3-1$ above. Therefore, $\frac{\partial \beta_{j}^{*}}{\partial \mu}>0$.

3-3. $\frac{\partial E(s)}{\partial \mu_{j}}=\frac{\partial w_{m c, 1}^{*} q\left(o+p \frac{w_{m c, 2}^{*}}{w_{m c, 1}^{*}}\right)}{\partial \mu_{j}} \lessgtr 0$ and $\frac{\partial E(k)}{\partial \mu_{j}}=\frac{\partial w_{m c, 1}^{*} q\left(s+t \frac{w_{m c, 2}^{*}}{w_{m c, 1}^{*}}\right)}{\partial \mu_{j}} \lessgtr 0$ by $3-1$.

3-4. $\frac{\partial E(w)}{\partial \mu_{j}}=\frac{\partial w_{o}+w_{m c, 1}^{*} E\left(s^{*}\right)+w_{m c, 2}^{*} E\left(k^{*}\right)}{\partial \mu_{j}}$ by 3-1 and 3-3 above. Hence, $\frac{\partial \frac{w_{0}}{E(w)}}{\partial \mu_{j}} \gtrless 0$.

3-5. $\frac{\partial \pi_{j}}{\partial \mu_{j}} \gtrless 0$ by $3-1,3-2,3-3$, and $3-4$.

4. Selling cost $c_{j}$

Let $w_{m c, 1}^{*}=\frac{a c+b}{2(c d+f)}, w_{m c, 2}^{*}=k\left(\frac{1}{2}-w_{m c, 1}^{*}\right), \frac{w_{m c, 2}^{*}}{w_{m c, 1}^{*}}=k\left(\frac{(c d+f)}{a c+b}-1\right)$.

4-1. $\frac{\partial w_{1 j}^{*}}{\partial c_{j}}=\frac{a f-b d}{2(d c+f)^{2}}<0, \quad \frac{\partial w_{2 j}^{*}}{\partial c_{j}}=-\frac{(a f-b d) k}{2(d c+f)^{2}}>0, \quad \frac{\partial \frac{w_{m c, 2}^{*}}{w_{m c, 1}^{*}}}{\partial c_{j}}=-\frac{(a f-b d) k}{(d c+f)^{2}}>0$ where $a f-b d<0$. If where $a f-b d>0$, the signs will be opposite. 
4-2. $\frac{\partial x_{j}^{*}}{\partial c_{j}}=-\frac{\left.\delta\left(a d c^{2}+2 b d c+b f\right)\right)}{2 c^{2}(d c+f)^{2}}<0$.

4-3. $\frac{\partial e_{1 j}^{*}}{\partial c_{j}}>0 \quad, \frac{\partial e_{2 j}^{*}}{\partial c_{j}}>0$ where $a f-b d<0$ by $4-1$ above. Therefore, $\frac{\partial \beta_{j}^{*}}{\partial c_{j}}>0$ where $a f-b d<0$.

4-4. $\frac{\partial E(s)}{\partial c_{j}}=-\frac{\delta\left(b f \delta+2 b d c \delta+c^{2}\left(a d \delta+o(b d-a f)+k p\left(d^{2} \delta-b d+f(a+d)\right)\right)\right)}{2 c^{2}(d c+f)^{2}}<0$, and $\frac{\partial E(k)}{\partial c_{j}}=-\frac{\delta\left(o(b d-a f)+k p\left(d^{2} \delta-b d+f(a+d)\right)\right)}{2(d c+f)^{2}}<0$.

4-5. $\frac{\partial E(w)}{\partial c_{j}}<0$ by $4-4$ above. Ergo, $\frac{\partial \frac{w_{0}}{E(w)}}{\partial c_{j}}>0$.

4-6. $\frac{\partial \pi_{j}}{\partial c_{j}}<0$ by 4-2, 4-4, and 4-5.

5. Thinking cost $c_{1 j}$ (similar to $\left.c_{2 j}\right)$

Let $w_{m c, 1}^{*}=\frac{a+b c_{1}}{2\left(c+c_{1} d\right.}, w_{m c, 2}^{*}=\frac{c_{1} f+g}{h+c_{1} k}\left(\frac{1}{2}-w_{m c, 1}^{*}\right), \frac{w_{m c, 2}^{*}}{w_{m c, 1}^{*}}=\frac{c_{1} f+g}{h+c_{1} k}\left(\frac{c+c_{1} d}{a+b c_{1}}-1\right)$.

5-1. $\frac{\partial w_{1 j}^{*}}{\partial c_{1 j}}=-\frac{a d-b c}{2\left(c+d c_{1}\right)^{2}}<0$, and $\frac{\partial w_{2 j}^{*}}{\partial c_{1 j}}=\frac{z}{2\left(c+d c_{1}\right)^{2}\left(h+k c_{1}\right)^{2}}>0$ where $z=\left(f c_{1}+\right.$ $g)\left(h+k c_{1}\right)(a d-b c)+k\left(d c_{1}+c\right)\left(f c_{1}+g\right)\left(a+c_{1}(b-d)-c\right)-f\left(d c_{1}+c\right)\left(k c_{1}+h\right)-\left(a+c_{1}(b-\right.$ $d)-c)$. Then, $\frac{\partial \frac{w_{m c, 2}^{*}}{w_{m c, 1}^{*}}}{\partial c_{1 j}}=\frac{1}{\left(h+k c_{1}\right)^{2}}\left(\frac{\left(f c_{1}+g\right)\left(h+k c_{1}\right)(a d-b c)}{\left(a+b c_{1}\right)^{2}}+\frac{f h\left(c+d c_{1}\right)}{a+b c_{1}}-\frac{g k\left(c+d c_{1}\right.}{a+b c_{1}}-f h+g c_{1}\right)>$ 0. Based on them, $\frac{\partial x_{j}^{*}}{\partial c_{1 j}}=-\frac{\delta\left(a d-b c_{1}\right)}{2 c\left(c+d c_{1}\right)^{2}}<0$.

5-2. $\frac{\partial e_{1 j}^{*}}{\partial c_{1 j}} \approx \frac{\partial \frac{w_{m c, 2}^{*}}{w_{m c, 1}^{*}}}{\partial c_{1 j}}<0 \quad, \frac{\partial e_{2 j}^{*}}{\partial c_{1 j}} \approx \frac{\partial \frac{w_{m c, 2}^{*}}{w_{m c, 1}^{*}}}{\partial c_{1 j}}<0$ by 5 -1 above. Therefore, $\frac{\partial \beta_{j}^{*}}{\partial c_{1 j}}<0$.

5-3. $\frac{\partial E(s)}{\partial c_{1 j}}<0$, and $\frac{\partial E(k)}{\partial c_{1 j}}<0$ by $5-1$ and $5-2$ above.

5-4. $\frac{\partial E(w)}{\partial c_{1 j}}<0$ by 5-1, 5-2, and 5-3 above. Ergo, $\frac{\partial \frac{w_{0}}{E(w)}}{\partial c_{1 j}}>0$.

$5-5 . \frac{\partial \pi_{j}}{\partial c_{1 j}}<0$ by $5-2,5-3$, and $5-2$.

6. Thinking effectiveness $\phi_{j}\left(\right.$ similar to $\left.u_{j}\right)$

Let $w_{m c, 1}^{*}=\frac{\phi_{j}^{2} a-\phi_{j} b+c}{2\left(\phi_{j}^{2} a-\phi_{j} b+d\right)}, w_{m c, 2}^{*}=\frac{\phi_{j} f+g}{h}\left(\frac{1}{2}-w_{m c, 1}^{*}\right), \frac{w_{m c, 2}^{*}}{w_{m c, 1}^{*}}=\frac{\phi_{j} f+g}{h}\left(\frac{\left(\phi_{j}^{2} a-\phi_{j} b+d\right)}{\phi_{j}^{2} a-\phi_{j} b+c}-1\right)$.

6-1. $\frac{\partial w_{1 j}^{*}}{\partial \phi_{j}}=\frac{(d-c)\left(2 \phi_{j}-b\right)}{2\left(\phi_{j}^{2}-b \phi_{j}+d\right)^{2}}>0\left(\because \phi_{j}>c u \gamma_{1} \gamma_{2}\right)$, and $\frac{\partial w_{2 j}^{*}}{\partial \phi_{j}}=-\frac{(d-c)\left(f \phi_{j}^{2}+2 g \phi_{j}-b g+d f\right)}{2 h\left(\phi_{j}^{2}-b \phi_{j}+d\right)^{2}}<$ $0, \quad \frac{\partial \frac{w_{m c, 2}^{*}}{w_{m c, 1}^{*}}}{\partial \phi_{j}}=-\frac{(d-c)\left(f \phi_{j}^{2}+2 g \phi_{j}-b g+c f\right)}{h\left(\phi_{j}^{2}-b \phi_{j}+c\right)^{2}}<0$. Based on them, $\frac{\partial x_{j}^{*}}{\partial \phi_{j}}=\frac{\left.(d-c)\left(2 \phi_{j}-b\right) \delta\right)}{2 c\left(\phi_{j}^{2}-b \phi_{j}+d\right)^{2}}>0$.

6-2. $\frac{\partial e_{1 j}^{*}}{\partial \phi_{j}} \approx \frac{\partial \frac{w_{m c, 2}^{*}}{w_{m c, 1}^{*}}}{\partial \phi_{j}}<0$ by 6-1. $\frac{\partial e_{2 j}^{*}}{\partial \phi_{j}} \approx \frac{\partial o \phi_{j}+p \frac{w_{m c, 2}^{*}}{w_{m c, 1}^{*}}}{\partial \phi_{j}}>0$. Also, $\frac{\partial \beta_{j}^{*}}{\partial \phi_{j}}>0$ based on Mathematica computation.

6-3. $\frac{\partial E(s)}{\partial \phi_{j}}>0$, and $\frac{\partial E(k)}{\partial \phi_{j}}>0$ by $6-1$ and $6-2$ above. 
6-4. $\frac{\partial E(w)}{\partial \phi_{j}} \lessgtr 0$ by 6-1, 6-2, and 6-3 above. Ergo, $\frac{\partial \frac{w_{0}}{E(w)}}{\partial \phi_{j}} \gtrless 0$.

6-5. $\frac{\partial \pi_{j}}{\partial \phi_{j}} \gtrless 0$ by $6-2,6-3$, and $6-2$.

7. Basic ability $\delta_{j}$

Let $w_{m c, 1}^{*}=\frac{a+b \delta(\delta-\nu)}{2(c+d \delta)}, w_{m c, 2}^{*}=k\left(\frac{1}{2}-w_{m c, 1}^{*}\right), \frac{w_{m c, 2}^{*}}{w_{m c, 1}^{*}}=k\left(\frac{(c+d \delta)}{a+b \delta(\delta-\nu)}-1\right)$.

7-1. $\frac{\partial w_{1 j}^{*}}{\partial \delta_{j}}=\frac{b d \delta_{j}^{2}+2 b c \delta_{j}-(b c \nu+a d)}{2\left(d \delta_{j}+c\right)^{2}} \lessgtr 0$, and $\frac{\partial w_{2 j}^{*}}{\partial \delta_{j}}=-\frac{k\left(b d \delta_{j}^{2}+2 b c \delta_{j}-(b c \nu+a d)\right)}{2\left(d \delta_{j}+c\right)^{2}} \lessgtr 0$, $\frac{\partial \frac{w_{m c, 2}^{*}}{w_{m c, 1}^{*}}}{\partial \delta_{j}}=-\frac{k\left(b\left(d \delta_{j}^{2}+2 c \delta_{j}-c \nu\right)-a d\right)}{\left(b \delta_{j}\left(\delta_{j}-\nu\right)+a\right)^{2}} \lessgtr 0$. Hence, $\frac{\partial x_{j}^{*}}{\partial \delta_{j}}=\frac{2 b d \delta_{j}^{3}+(3 b c-b d \nu) \delta_{j}^{2}-2 b c \nu \delta_{j}+a c}{2 g\left(d \delta_{j}+c\right)^{2}}>0$.

7-2. $\frac{\partial e_{1}^{*}}{\partial \delta_{j}}=-\frac{1}{\delta_{j}^{2}}\left(y-\frac{k j\left(b\left(d \delta_{j}^{2}+2 c \delta_{j}-c \nu\right)-a d\right)}{\left(b \delta_{j}\left(\delta_{j}-\nu\right)+a\right)^{2}}\right)<0 \quad$ by 7-1. Similarly, $\frac{\partial e_{2 j}^{*}}{\partial \delta_{j}}<0$. Also, $\frac{\partial \beta_{j}^{*}}{\partial \delta_{j}} \lessgtr 0$ because $\beta_{j}^{*}=\delta_{j}+\frac{1}{\delta_{j}}\left(s+t \frac{w_{m c, 2}^{*}}{w_{m c, 1}^{*}}\right)$.

7-3. $\frac{\partial E(s)}{\partial \delta_{j}}>0$, and $\frac{\partial E(k)}{\partial \delta_{j}} \lessgtr 0$ by $7-1$ and $7-2$ above.

7-4. $\frac{\partial E(w)}{\partial \delta_{j}} \lessgtr 0$ by 7-1, 7-2, and 7-3 above. Ergo, $\frac{\partial \frac{w_{0}}{E(w)}}{\partial \delta_{j}} \gtrless 0$.

7-5. $\frac{\partial \pi_{j}}{\partial \delta_{j}} \gtrless 0$ by $7-2,7-3$, and $7-2$.

\section{Cost of Activity $\nu$}

Let $w_{m c, 1}^{*}=\frac{a+b \delta(\delta-\nu)}{2 c}, w_{m c, 2}^{*}=d\left(\frac{1}{2}-w_{m c, 1}^{*}\right), \frac{w_{m c, 2}^{*}}{w_{m c, 1}^{*}}=d\left(\frac{(c+d \delta)}{a+b \delta(\delta-\nu)}-1\right)$.

8-1. $\frac{\partial w_{1 j}^{*}}{\partial \nu}=-\frac{b \delta_{j}}{2 c}<0$, and $\quad \frac{\partial w_{2 j}^{*}}{\partial \nu}=\frac{d \delta_{j}}{2 c}>0, \quad \frac{\partial \frac{w_{m c, 2}^{*}}{w_{m c, 1}^{*}}}{\partial \nu}=\frac{c d \delta_{j}}{\left(\delta\left(\nu-b \delta_{j}\right)-a\right)^{2}}>0$. Hence, $\frac{\partial x_{j}^{*}}{\partial \nu}=-\frac{b \delta_{j}^{2}}{2 c^{2}}<0$.

8-2. $\frac{\partial e_{1 j}^{*}}{\partial \nu} \approx \frac{\partial \frac{w_{m c, 2}^{*}}{w_{m c, 1}^{*}}}{\partial \nu}>0$ and $\frac{\partial e_{2 j}^{*}}{\partial \nu} \approx \frac{\partial \frac{w_{m c, 2}^{*}}{w_{m c, 1}^{*}}}{\partial \nu}>0$ by 8-1. Hence, $\frac{\partial \beta_{j}^{*}}{\partial \nu}>0$.

8-3. $\frac{\partial E(s)}{\partial \nu}=\frac{(f d-h) \delta_{j}}{2 c} \lessgtr 0$, and $\frac{\partial E(k)}{\partial \nu}=\frac{(s d-f) \delta_{j}}{2 c} \lessgtr 0$ by 8-1 and 8-2 above.

8-4. $\frac{\partial E(w)}{\partial \nu} \lessgtr 0$ by 8-1, 8-2, and 8-3 above. Ergo, $\frac{\partial \frac{w_{0}}{E(w)}}{\partial \nu} \gtrless 0$.

8-5. $\frac{\partial \pi_{j}}{\partial \nu} \gtrless 0$ by $8-2,8-3$, and $8-2$. 


\subsection{Optimization of New Model with Adaptable Selling Ef- fectiveness}

\subsubsection{Standard-Customized plan}

Given assumptions A1-A11 in section 3 and using the moment generating function of the normal distribution, the salesperson's expected net utility maximization problem is shown in section 3. Equation 3.5 can be restated as maximizing the certainty equivalent expression shown below:

$$
C E_{j}=w_{0}+w_{1 j} S\left(x_{j}\right)-\left(\frac{c_{1 j}\left(e_{1 j} x_{j}\right)^{2}}{2}+\frac{c_{2 j}\left(e_{2 j} x_{j}\right)^{2}}{2}+\frac{c_{j} x_{j}^{2}}{2}\right)-\frac{\mu_{j}}{2} w_{1 j}^{2} \sigma_{s j}^{2}
$$

The salespeople will maximizes their certainty equivalent with respect to $x, e_{1}, e_{2}$. Then the their efforts can be derived by taking derivatives by $x_{j}, e_{1 j}$, and $e_{2 j}$.

$$
x=\frac{w_{1 j}\left(\delta_{j}+u_{j} e_{1 j}+\phi_{j} e_{2 j}\right)}{c_{1 j} e_{1 j}^{2}+c_{2 j} e_{2 j}^{2}+c_{j}}, e_{1 j}=\frac{w_{1 j} u_{j}}{c_{1 j} x_{j}}, e_{2 j}=\frac{w_{1 j} \phi_{j}}{c_{2 j} x_{j}}(3)
$$

Both first and second order conditions are satisfied $\left(\frac{\partial C E_{j}}{\partial x_{j}}>0, \frac{\partial C E_{j}}{\partial x_{1 j}}>0, \frac{\partial C E_{j}}{\partial e_{2 j}}>0\right.$ and $\left.\frac{\partial^{2} C E_{j}}{\partial^{2} x_{j}}<0, \frac{\partial^{2} C E_{j}}{\partial^{2} x_{1 j}}<0, \frac{\partial^{2} C E_{j}}{\partial^{2} e_{2 j}}<0\right)$.

Based on equation 7.2, I can achieve the sales rep's strategy (IC constraint):

$$
x_{j}=\frac{w_{1 j} \delta_{j}}{c_{j}} \quad e_{1 j}=\frac{u_{j} c_{j}}{c_{1 j} \delta_{j}} \quad e_{2 j}=\frac{\phi_{j} c_{j}}{c_{2 j} \delta_{j}}
$$

Consequently, firm will choose a customized incentive to optimize their certainty equivalent (e.g., Misra et al. 2005). Firm's Certainty Equivalent (FCE):

$$
F C E_{j}=S_{j}-v x_{j}-w_{0 j}-w_{1 j} S_{j}-\left(\frac{c_{1 j}\left(e_{1 j} x_{j}\right)^{2}}{2}+\frac{c_{2 j}\left(e_{2 j} x_{j}\right)^{2}}{2}+\frac{c_{j} x_{j}^{2}}{2}\right)-\frac{\mu_{j}}{2} w_{1 j}^{2} \sigma_{s j}^{2}
$$


By IR constraint (equation 3.4), customized individual base salary $w_{0 j}$ can be represented as following:

$$
w_{0 j}=\bar{F}_{j}-w_{1 j} S_{j}+\left(\frac{c_{1 j}\left(e_{1 j} x_{j}\right)^{2}}{2}+\frac{c_{2 j}\left(e_{2 j} x_{j}\right)^{2}}{2}+\frac{c_{j} x_{j}^{2}}{2}\right)+\frac{\mu_{j}}{2} w_{1 j}^{2} \sigma_{s j}^{2}
$$

By replacing $w_{0 j}$ with equation 7.5 , the equation 7.4 can be shown as following:

$$
\begin{array}{r}
F C E_{j}=S_{j}-v x_{j}-\bar{F}_{j}-\left(c_{1 j}\left(e_{1 j} x_{j}\right)^{2}+c_{2 j}\left(e_{2 j} x_{j}\right)^{2}+c_{j} x_{j}^{2}\right)-\mu_{j} w_{1 j}^{2} \sigma_{s j}^{2} \\
=-\bar{F}_{j}+\alpha_{j}+x_{j}\left(\delta_{j}+u_{j} e_{1 j}+\phi_{j} e_{2 j}-v\right)-\left(c_{1 j} e_{1 j}^{2}+c_{2 j} e_{2 j}^{2}+c_{j}\right) x_{j}^{2}-\mu_{j} w_{1 j}^{2} \sigma_{s j}^{2}
\end{array}
$$

Finally, $x_{j}, 2_{1 j}$, and $e_{2 j}$ can be replaced by equation (7.3:IC constraint). Then the FCE can be represented as following:

$$
\begin{gathered}
F C E_{j}=-\bar{F}_{j}+\alpha_{j}+\frac{w_{1 j} \delta_{j}}{c_{j}}\left(\delta_{j}+\frac{u_{j}^{2} c_{j}}{c_{1 j} \delta_{j}}+\frac{\phi_{j}^{2} c_{j}}{c_{2 j} \delta_{j}}-v\right) \\
-\left(c_{1 j}\left(\frac{u_{j} c_{j}}{c_{1 j} \delta_{j}}\right)^{2}+c_{2 j}\left(\frac{\phi_{j} c_{j}}{c_{2 j} \delta_{j}}\right)^{2}+c_{j}\right)\left(\frac{w_{1 j} \delta_{j}}{c_{j}}\right)^{2}-\mu_{j} w_{1 j}^{2} \sigma_{s j}^{2}
\end{gathered}
$$

Take a derivative by $w_{1 j}$ :

$$
\begin{array}{r}
\frac{\partial F C E}{\partial w_{1 j}}=\frac{\delta_{j} c_{1 j} c_{2 j}\left(\delta_{j}-v\right)+c_{j}\left(c_{1 j} \phi_{j}^{2}+c_{2 j} u_{j}^{2}\right)}{c_{j} c_{1 j} c_{2 j}}-2 w_{1 j} \frac{c_{1 j} c_{2 j} \delta_{j}^{2}+c_{j}\left(c_{1 j} \phi_{j}^{2}+c_{2 j} u_{j}^{2}\right)}{c_{j} c_{1 j} c_{2 j}} \\
s-2 \mu_{j} w_{1 j} \sigma_{s j}^{2}=0
\end{array}
$$

Therefore, the optimal incentive under the customized Compensation is as following:

$$
w_{1 j}^{*}=\frac{\delta_{j} c_{1 j} c_{2 j}\left(\delta_{j}-v\right)+c_{j}\left(c_{j 1} \phi_{j}^{2}+c_{2 j} u_{j}^{2}\right)}{2\left(c_{j} c_{1 j} c_{2 j} \mu_{j} \sigma_{s j}^{2}+c_{1 j} c_{2 j} \delta_{j}^{2}+c_{j}\left(c_{1 j} \phi_{j}^{2}+c_{2 j} u_{j}^{2}\right)\right)}
$$

Also, sales rep's optimal strategy is as following:

$$
x_{j}^{*}=\frac{w_{1 j}^{*} \delta_{j}}{c_{j}} \quad e_{1 j}^{*}=\frac{u_{j} c_{j}}{c_{1 j} \delta_{j}} \quad e_{2 j}^{*}=\frac{\phi_{j} c_{j}}{c_{2 j} \delta_{j}}
$$


Reservation Utility :

$$
\bar{F}_{j}=w_{0 j}^{*}+w_{1 j}^{*} S_{j}-\left(\frac{c_{1 j}\left(e_{1 j}^{*} x_{j}^{*}\right)^{2}}{2}+\frac{c_{2 j}\left(e_{2 j}^{*} x_{j}^{*}\right)^{2}}{2}+\frac{c_{j} x_{j}^{*} 2}{2}\right)-\frac{\mu_{j}}{2} w_{1}^{*} 2 \sigma_{s j}^{2}
$$

Basic Salary:

$$
w_{0}^{*}=\bar{F}_{j}-w_{1 j}^{*} S_{j}+\left(\frac{c_{1 j}\left(e_{1 j}^{*} x_{j}^{*}\right)^{2}}{2}+\frac{c_{2 j}\left(e_{2 j}^{*} x_{j}^{*}\right)^{2}}{2}+\frac{c_{j} x_{j}^{*} 2}{2}\right)+\frac{\mu_{j}}{2} w_{1}^{* 2} \sigma_{s j}^{2}
$$

Risk Premium:

$$
R P_{j}=\frac{\mu_{j}}{2} w_{1}^{* 2} \sigma_{s j}^{2}
$$

\subsubsection{Multidimensional-Customized plan}

Sales reps' Certainty Equivalent under MC plan:

$C E_{j}=w_{0}+w_{1 j} S_{j}-w_{2 j} K_{j}-\left(\frac{c_{1 j}\left(e_{1 j} x_{j}\right)^{2}}{2}+\frac{c_{2 j}\left(e_{2 j} x_{j}\right)^{2}}{2}+\frac{c_{j} x_{j}^{2}}{2}\right)-\frac{\mu_{j}}{2} w_{1 j}^{2} \sigma_{s j}^{2}-\frac{\mu_{j}}{2} w_{2 j}^{2} \sigma_{k j}^{2}$

where $S_{j}=\alpha_{j}+x_{j}\left(\delta_{j}+u_{j} e_{1 j}+\phi_{j} e_{2 j}\right)$, and $K_{j}=\gamma_{j}\left(e_{1 j}+e_{2 j}\right) x_{j}$.

By taking derivatives $\left(\frac{\partial C E_{j}}{\partial x_{j}}, \frac{\partial C E_{j}}{\partial e_{1 j}}, \frac{\partial C E_{j}}{\partial e_{2 j}}\right)$, I can confirm that the first and second order conditions are satisfied. Then, I can obtain reps' strategy will be a function of $w_{1 j}$ and $w_{2 j}$ :

$$
x_{j}=\frac{w_{1 j} \delta_{j}}{c_{j}} \quad e_{1 j}=\frac{c_{j}\left(w_{1 j} u_{j}+w_{2 j} \gamma_{j}\right)}{c_{1 j} w_{1 j} \delta_{j}} \quad e_{2 j}=\frac{c_{j}\left(w_{1 j} \phi_{j}+w_{2 j} \gamma_{j}\right)}{c_{2 j} w_{1 j} \delta_{j}}
$$


The firm's CE is with IR and IC conditions ban be shown as:

$$
\begin{array}{r}
F C E_{j}=-\bar{F}_{j}+\alpha_{j}+\frac{\left(\delta_{j}-v\right) w_{1 j} \delta_{j}}{c_{j}}+\frac{u_{j}\left(w_{1 j} u_{j}+w_{2 j} \gamma_{j}\right)}{c_{1 j}} \\
+\frac{\phi_{j}\left(w_{1 j} \phi_{j}+w_{2 j} \gamma_{j}\right)}{c_{2 j}}-\frac{w_{1 j}^{2} u_{j}^{2}+w_{2 j}^{2} \gamma_{j}^{2}+2 w_{1 j} w_{2 j} u_{j} \gamma_{j}}{c_{1 j}}-\frac{w_{1 j}^{2} \phi_{j}^{2}+w_{2 j}^{2} \gamma_{j}^{2}+2 w_{1 j} w_{2 j} \phi_{j} \gamma_{j}}{c_{2 j}} \\
-\frac{w_{1 j}^{2} \delta_{j}^{2}}{c_{j}}-\mu_{j} w_{1 j}^{2} \sigma_{s j}^{2}-\mu_{j} w_{1 j}^{2} \sigma_{k j}^{2}
\end{array}
$$

Take a derivative by $w_{1 j}$ and $w_{2 j}$, I can achieve the closed form of optimal incentives in MC plan.

$$
w_{1 j}^{*}=\frac{\alpha_{j}+\delta_{j}\left(\delta_{j}-v_{j}\right) b_{j}}{2\left(\alpha_{j}+h_{j} b_{j}\right)}, w_{2 j}^{*}=\frac{k_{j}}{b_{2 j}}\left(\frac{1}{2}-w_{1 j}^{*}\right)
$$

where $\alpha_{j}=c_{j}\left[\left(\phi_{j} \gamma_{1 j}-u_{j} \gamma_{2 j}\right)^{2}+\left(c_{1 j} \phi_{j}^{2}+c_{2 j} u_{j}^{2}\right) \mu_{j} \sigma_{K j}^{2}\right], b_{j}=\gamma_{1 j}^{2} c_{2 j}+\gamma_{2 j}^{2} c_{1 j}+\mu_{j} \sigma_{K j}^{2} c_{1 j} c_{2 j}$, and $h_{j}=\delta_{j}^{2}+\mu_{j} \sigma_{S j}^{2} c_{j}$, and $k_{j}=\gamma_{2 j} c_{1 j} \phi_{j}+\gamma_{1 j} c_{2 j} u_{j}$. And the sales rep's optimal effort strategy is as following:

$$
x_{j}^{*}=\frac{w_{1 j}^{*} \delta_{j}}{c_{j}} \quad e_{1 j}^{*}=\frac{c_{j}\left(w_{1 j}^{*} u_{j}+w_{2 j}^{*} \gamma_{j}\right)}{c_{1 j} w_{1 j}^{*} \delta_{j}} \quad e_{2 j}^{*}=\frac{c_{j}\left(w_{1 j}^{*} \phi_{j}+w_{2 j}^{*} \gamma_{j}\right)}{c_{2 j} w_{1 j}^{*} \delta_{j}}
$$

\subsubsection{Standard-Uniform plan}

Under the standard-uniform plan, sales reps' optimal strategy is the similar form of standard-customized one (equation 7.3).

However, the firm's FCE is different because the firm will choose the $\bar{w}_{1}$ for all heterogeneous sales force. Hence, it can be written as follow:

$$
F C E=\sum_{j=1}^{J}\left[\bar{F}_{j}+\alpha_{j}+\bar{w}_{1} \frac{\ell+c_{j} z}{c_{j} c_{1 j} c_{2 j}}-\bar{w}_{1}{ }^{2} \frac{c_{1 j} c_{2 j} \delta_{j}^{2}+c_{j} z}{c_{j} c_{1 j} c_{2 j}}-\mu_{j} \bar{w}_{1}^{2} \sigma_{j}^{2}\right]
$$


where $\ell=\delta_{j} c_{1 j} c_{2 j}\left(\delta_{j}-v\right), \quad z=c_{1 j} \phi_{j}^{2}+c_{2 j} u_{j}^{2}$. To obtain the firm's optimal incentives and reps' optimal choices, I take the derivative by $\bar{w}_{1}\left(\frac{\partial F C E}{\partial \bar{w}_{1}}\right)$. Hence, the optimal incentive under the SU Compensation is $\overline{w_{1}^{*}}=\frac{\sum_{j=1}^{J} \frac{\delta_{j} c_{1 j} c_{2 j}\left(\delta_{j}-v\right)+c_{j}\left(c_{j 1} \phi_{j}^{2}+c_{2 j} u_{j}^{2}\right)}{c_{j} c_{1 j} c_{2 j}}}{2\left[\sum_{j=1}^{J} \frac{c_{1 j} c_{2 j} \delta_{j}^{2}+c_{j}\left(c_{1 j} \phi_{j}^{2}+c_{2 j} u_{j}^{2}\right)}{c_{j} c_{1 j} c_{2 j}}+\sum_{j=1}^{J} \mu_{j} \sigma_{s j}^{2}\right]}$, and the reps' optimal effort choices are $x_{j}^{*}=\frac{\bar{w}_{1}^{*} \delta_{j}}{c_{j}}, e_{1 j}^{*}=\frac{u_{j} c_{j}}{c_{1 j} \delta_{j}}$, and $e_{2 j}^{*}=\frac{\phi_{j} c_{j}}{c_{2 j} \delta_{j}}$.

Reservation utility, basic salary, and risk premium can be derived by inserting the optimal incentive under SU plan into same equations of 7.10, 7.11, eqn:rp1.

\subsubsection{Multidimensional-Uniform plan}

Since reps' CE is equal to MC pan case, reps' choices (IC constraint) are same with the equation 7.14 under the MC plan. Then, firm's CE from all rep is is as following:

$$
\begin{array}{r}
F C E_{m u}=\sum_{j=1}^{J}\left[\bar{F}_{j}+\alpha_{j}+\frac{\left(\delta_{j}-v\right) \bar{w}_{1} \delta_{j}}{c_{j}}+\frac{u_{j}\left(\bar{w}_{1} u_{j}+\bar{w}_{2} \gamma_{1 j}\right)}{c_{1 j}}\right. \\
+\frac{\phi_{j}\left(\bar{w}_{1} \phi_{j}+\bar{w}_{2} \gamma_{2 j}\right)}{c_{2 j}}-\frac{\bar{w}_{1}^{2} u_{j}^{2}+\bar{w}_{2}^{2} \gamma_{1 j}^{2}+2 \bar{w}_{1} \bar{w}_{2} u_{j} \gamma_{1 j}}{c_{1 j}} \\
\left.-\frac{\bar{w}_{1}^{2} \phi_{j}^{2}+\bar{w}_{2}^{2} \gamma_{2 j}^{2}+2 \bar{w}_{1} \bar{w}_{2} \phi_{j} \gamma_{2 j}}{c_{2 j}}-\frac{\bar{w}_{1}^{2} \delta_{j}^{2}}{c_{j}}-\mu_{j} \bar{w}_{1}^{2} \sigma_{s j}^{2}-\mu_{j} \bar{w}_{2}^{2} \sigma_{k j}^{2}\right]
\end{array}
$$

By F.O.C,$\frac{\partial F C E}{\partial \bar{w}_{1}}=0$ and $\frac{\partial F C E}{\partial \bar{w}_{2}}=0$, the firm's optimal incentives $\overline{w_{1}^{*}}, \bar{w}_{2}^{*}$ for Uniform plan are as following:

$$
\bar{w}_{1}^{*}=\frac{\sum_{j=1}^{J} \lambda_{j} \sum_{j=1}^{J} \eta_{j}-\left(\sum_{j=1}^{J} \theta_{j}\right)^{2}}{\left.2\left(\sum_{j=1}^{J} \rho_{j} \sum_{j=1}^{J} \eta_{j}-\left(\sum_{j=1}^{J} \theta_{j}\right)^{2}\right)\right)}, \bar{w}_{2}^{*}=\frac{\sum_{j=1}^{J} \theta_{j}}{\sum_{j=1}^{J} \eta_{j}}\left(\frac{1}{2}-\overline{w_{1}^{*}}\right)
$$

where $\lambda_{j}=\frac{\left(\delta_{j}-v\right) \delta_{j} c_{1 j} c_{2 j}+c_{j}\left(c_{2 j} u_{j}^{2}+c_{1 j} \phi_{j}^{2}\right)}{c_{j} c_{1 j} c_{2 j}}, \eta_{j}=\frac{\left(c_{1 j} \gamma_{2 j}^{2}+c_{2 j} \gamma_{1 j}^{2}\right)}{c_{1 j} c_{2 j}}+\mu_{j} \sigma_{K j}^{2}, \theta_{j}=\frac{\left(\gamma_{1 j} c_{2 j} u_{j}+\gamma_{2 j} c_{1 j} \phi_{j}\right)}{c_{1 j} c_{2 j}}$, and

$\rho_{j}=\frac{c_{j}\left(c_{2 j} u_{j}^{2}+c_{1 j} \phi_{j}^{2}\right)+\delta_{j}^{2} c_{1 j} c_{2 j}+c_{j} c_{1 j} c_{2 j} \mu_{j} \sigma_{s j}^{2}}{c_{j} c_{1 j} c_{2 j}}$. The reps' optimal selling and thinking efforts can be represented by inserting the optimal incentives (equation 7.20) into equation 7.14 . 


\subsection{Comparative Statics Proofs for Essay 2}

\subsubsection{Derivation of Certainty Equivalent under Target-Value Wage}

Under the Target-Value wage, a rep's certainty equivalent can be derived in this way.

As an example, we will show ISR's expected utility function as below:

$$
E\left[U_{i}\right]=E\left[1-e^{-\mu\left(\gamma+w_{1} \varepsilon_{i 1}+w_{2} \varepsilon_{i 2}+\theta \varepsilon_{o 2}-w_{4} \varepsilon_{o 2}^{2}\right)}\right]
$$

where $\gamma=w_{0}+w_{1} s_{i 1}+w_{2} s_{i 2}+w_{3} k_{i}-w_{4}\left(s_{o 2}-q_{i}\right)^{2}-D_{i}, \theta=2 w_{4}\left(s_{o 2}-q_{i}\right)$, and $z=\frac{\sigma_{o 2}^{2}}{1-2 \mu \sigma_{o 2}^{2} w_{4}}$.

$$
\begin{array}{r}
E\left[U_{i}\right]=1-\iiint e^{-\mu\left(\gamma+w_{1}\left(\varepsilon_{i 1}+\varepsilon_{i 2}\right)+\theta \varepsilon_{o 2}-w_{3} \varepsilon_{o 2}^{2}\right)} \frac{1}{\sqrt{2 \pi \sigma_{o 2}^{2}}} e^{-\frac{\varepsilon_{o 2}^{2}}{2 \sigma_{o 2}^{2}}} \\
\frac{1}{\sqrt{2 \pi \sigma_{i 1}^{2} w_{1}^{2}}} e^{-\frac{(a-\mu)^{2}}{2 w_{1}^{2} \sigma_{i 1}^{2}}} \frac{1}{\sqrt{2 \pi \sigma_{i 2}^{2} w_{1}^{2}}} e^{-\frac{(a-\mu)^{2}}{2 w_{1}^{2} \sigma_{i 2}^{2}}} d_{\varepsilon_{o 2}} d_{\varepsilon_{i 1} d_{\varepsilon_{i 2}}}
\end{array}
$$

Let $x=\frac{1}{\sqrt{2 \pi \sigma_{i 1}^{2} w_{1}^{2}}} e^{-\frac{(a-\mu)^{2}}{2 w_{1}^{2} \sigma_{i 1}^{2}}} \frac{1}{\sqrt{2 \pi \sigma_{i 2}^{2} w_{1}^{2}}} e^{-\frac{(a-\mu)^{2}}{2 w_{1}^{2} \sigma_{i 2}^{2}}}$. Then,

$$
\begin{aligned}
& E\left[U_{i}\right]=1-\iint \frac{1}{\sqrt{2 \pi \sigma_{o 2}^{2}}} e^{-\mu\left(\gamma+w_{1}\left(\varepsilon_{i 1}+\varepsilon_{i 2}\right)\right)} x \int e^{-\mu \theta \varepsilon_{o 2} 2} e^{-\varepsilon_{o 2}^{2} \frac{1-2 \mu \sigma_{o 2}^{2} w_{3}}{2 \sigma_{o 2}^{2}}} d_{\varepsilon_{o 2}} d_{\varepsilon_{i 1}} d_{\varepsilon_{i 2}} \\
& =1-\iint \sqrt{\frac{z^{2}}{\sigma_{o 2}^{2}}} e^{-\mu\left(\gamma+w_{1}\left(\varepsilon_{i 1}+\varepsilon_{i 2}\right)\right)} x \int e^{-\mu \theta \varepsilon_{o 2}} \frac{1}{z \sqrt{2 \pi}} e^{-\frac{\varepsilon_{o 2}^{2}}{2 z}} d_{\varepsilon_{o 2}} d_{\varepsilon_{i 1}} d_{\varepsilon_{i 2}} \\
& =1-\iint e^{\frac{1}{2}\left(\ln z^{2}-\ln \sigma_{o 2}^{2}\right)} e^{-\mu \gamma} e^{\mu^{2} \theta^{2} \frac{z}{2}} e^{-\mu w_{1}\left(\varepsilon_{i 1}+\varepsilon_{i 2}\right)} x d_{\varepsilon_{i 1}} d_{\varepsilon_{i 2}} \\
& =1-\iint e^{-\mu\left[\frac{1}{2 \mu} \ln \left(1-2 \mu \sigma_{o 2}^{2} w_{3}\right)+\gamma-\mu \theta^{2} \frac{z}{2}\right]} e^{-\mu w_{1}\left(\varepsilon_{i 1}+\varepsilon_{i 2}\right)} x d_{\varepsilon_{i 1}} d_{\varepsilon_{i 2}}
\end{aligned}
$$


Let $y=\left[\frac{1}{2 \mu} \ln \left(1-2 \mu \sigma_{o 2}^{2} w_{3}\right)+\gamma-\mu \theta^{2} \frac{z}{2}\right]$.

$$
\begin{gathered}
=1-\iint e^{-\mu\left(y+w_{1} \varepsilon_{i 1}\right)} \frac{1}{\sqrt{2 \pi \sigma_{i 1}^{2}}} e^{-\frac{w_{1}^{2} \varepsilon_{i 1}^{2}}{2 w_{1}^{2} \sigma_{i 1}^{2}}} e^{-\mu\left(y+w_{1} \varepsilon_{i 2}\right)} \frac{1}{\sqrt{2 \pi \sigma_{i 2}^{2}}} e^{-\frac{w_{1}^{2} \varepsilon_{i 2}^{2}}{2 w_{1}^{2} \sigma_{i 2}^{2}}} d_{\varepsilon_{i 1}} d_{\varepsilon_{i 2}} \\
=1-e^{-\mu\left(y-\frac{\mu}{2} w_{1}^{2} \sigma_{i 1}^{2}-\frac{\mu}{2} w_{1}^{2} \sigma_{i 2}^{2}\right)} \\
=1-e^{-\mu\left(\frac{1}{2 \mu} \ln \left(1-2 \mu \sigma_{o 2}^{2} w_{4}\right)+w_{o}+w_{1} s_{i 1}+w_{2} s_{i 2}+w_{3} k_{i}-w_{4}\left(s_{o 1}-q_{i}\right)^{2}-D_{i}-\mu \theta^{2} \frac{z}{2}-\frac{\mu}{2} w_{1}^{2} \sigma_{i 1}^{2}-\frac{\mu}{2} w_{2}^{2} \sigma_{i 2}^{2}\right.}
\end{gathered}
$$

Therefore,

$$
\begin{array}{r}
C E_{i}=w_{o}+\frac{1}{2 \mu} \ln \left(1-2 \mu \sigma_{o 2}^{2} w_{4}\right)+w_{1} s_{i 1}+w_{2} s_{i 2}+w_{3} k_{i} \\
-w_{4}\left(s_{o 1}-q_{i}\right)^{2}-\frac{2 w_{3}^{2} \mu \sigma_{o 2}^{2}\left(s_{o 2}-q_{i}\right)^{2}}{1-2 w_{3} \mu \sigma_{o 2}^{2}}-\frac{\mu}{2}\left(w_{1}^{2} \sigma_{i 1}^{2}+w_{2}^{2} \sigma_{i 2}^{2}\right)-D_{i}
\end{array}
$$

\subsection{Optimization of Target-Value Compensation}

The ISR's Certainty Equivalent is as below;

$$
\begin{aligned}
C E_{i}=w_{0}+\frac{1}{2 \mu} \ln \left(1-2 \mu \sigma_{o 2}^{2} w_{3}\right) & -\frac{\mu}{2}\left(w_{1}^{2} \sigma_{i 1}^{2}+w_{2}^{2} \sigma_{i 2}^{2}\right)+w_{1} s_{i 1}+w_{2} s_{i 2}+w_{3} k_{i o} \\
& -\frac{w_{4}\left(\Omega_{i}\right)^{2}}{1-2 \mu \sigma_{o 2}^{2} w_{3}}-\left(\frac{c_{i i s} e_{i i s}^{2}}{2}+\frac{c_{i o s} e_{i o s}^{2}}{2}+\frac{c_{i q} e_{i q}^{2}}{2}\right)
\end{aligned}
$$

When we take the first order condition regarding each effort choice, 


$$
\begin{aligned}
& \frac{\partial C E_{i}}{\partial e_{i i s}}=w_{1} \beta_{i i s}+w_{2} \beta_{i i s} \Omega_{o}-c_{i i s} e_{i i s}=0 \rightarrow e_{i i s}=\frac{w_{1} \beta_{i i s} p_{i}}{c_{i i s}} \\
& \frac{\partial C E_{i}}{\partial e_{i q}}=w_{3} \beta_{i q}-c_{i q} \beta_{i q}=0 \rightarrow e_{i q}=\frac{w_{3} \beta_{i q} p_{o}}{c_{i q}} \\
& \frac{\partial C E_{i}}{\partial e_{i o s}}=w_{2} \beta_{i o s} \beta_{o q} e_{o q}-c_{i o s} e_{i o s}=0 \rightarrow e_{i o s}=\frac{w_{2} v_{3} \beta_{i o s} \beta_{o q}^{2} p_{i}^{2}}{c_{i o s} c_{o q}} \\
& \frac{\partial C E_{i}}{\partial \Omega_{i}}=w_{3}-2 w_{4} \Omega_{i}=0 \rightarrow \Omega_{i}=\frac{w_{3}}{p_{o}^{2}}\left(\frac{1}{2 w_{4}}-\mu \sigma_{i 2}^{2}\right)
\end{aligned}
$$

Firm's Certainty Equivalent is as below;

$$
\begin{array}{r}
F C E=-\bar{F}_{i}-\bar{F}_{o}-\frac{\sigma^{2}}{2}-\mu\left(w_{1}^{2} \sigma_{i 1}^{2}+w_{2}^{2} \sigma_{i 2}^{2}\right)-\rho\left(v_{1}^{2} \sigma_{o 1}^{2}+v_{2}^{2} \sigma_{o 2}^{2}\right)+h_{i}+h_{o} \\
-\frac{w_{1}^{2} \beta_{i i s}^{2} p_{i}^{2}}{2 c_{i i s}}-\frac{w_{2}^{2} v_{3}^{2} \beta_{i o s}^{2} \beta_{o q}^{4} p_{i}^{4}}{2 c_{i o s} c_{o q}^{2}}-\frac{w_{3}^{2} \beta_{i q}^{2} p_{o}^{2}}{2 c_{i q}}-\frac{v_{1}^{2} \beta_{o o s}^{2} p_{o}^{2}}{2 c_{o o s}}-\frac{v_{2}^{2} w_{3}^{2} \beta_{o i s}^{2} \beta_{i q}^{4} p_{o}^{4}}{2 c_{o i s} c_{i q}^{2}}-\frac{v_{3}^{2} \beta_{o q}^{2} p_{i}^{2}}{2 c_{o q}} \\
+\frac{w_{1} \beta_{i i s}^{2} p_{i}^{2}}{c_{i i s}}+\frac{w_{2} v_{3}^{2} \beta_{i o s}^{2} \beta_{o q}^{4} p_{i}^{3}}{c_{o q}^{2} c_{i o s}}+\frac{v_{1} \beta_{o o s}^{2} p_{o}^{2}}{c_{o o s}}+\frac{v_{2} w_{3}^{2} \beta_{o i s}^{2} \beta_{i q}^{4} p_{o}^{3}}{c_{o i s} c_{i q}^{2}} \\
-\frac{1}{p_{o}^{2}\left(1-2 w_{4} \mu \sigma_{i 2}^{2}\right)}\left(w_{3}^{2} \mu \sigma_{i 2}^{2}+4 w_{3}^{2} w_{4}^{2} \mu^{3} \sigma_{i 2}^{6}-4 w_{3}^{2} w_{4} \mu^{2} \sigma_{i 2}^{4}\right) \\
-\frac{1}{p_{i}^{2}\left(1-2 v_{4} \rho \sigma_{o 2}^{2}\right)}\left(v_{3}^{2} \rho \sigma_{o 2}^{2}+4 v_{3}^{2} v_{4}^{2} \rho^{3} \sigma_{o 2}^{6}-4 v_{3}^{2} v_{4} \rho^{2} \sigma_{o 2}^{4}\right)
\end{array}
$$

By taking derivatives, we can obtain the optimal incentives. 


\section{VITA}

Yeji Lim was born on October $17^{\text {th }}, 1984$ in Seoul, South Korea. She attended Sookmyoung High School, Seoul, South Korea. In 2003, she entered Kyungwon University, and graduated with a Bachelor's degree in Management in June, 2007. She received a degree of Master of Economics from Sungkyunkwan University in February, 2012. In January 2013, she started the MBA program at the University of Missouri-Columbia and also entered the Ph.D. program at the same school in August

2013 during her MBA degree. She received MBA degree in May 2016 and Ph.D. in Marketing from the University of Missouri-Columbia. 